\title{
Propriedade de Bishop-Phelps-Bollobás
}

\author{
Juliane Trianon Fraga \\ DissERTAÇÃO APRESENTADA \\ $\mathrm{AO}$ \\ Instituto DE MatemáticA E Estatística \\ DA \\ Universidade DE SÃo PAUlo \\ PARA \\ OBTENÇÃO DO TÍTULO \\ DE \\ Mestre em MatemáticA \\ Programa: Matemática \\ Orientador: Prof ${ }^{\mathrm{a}}$. Dr ${ }^{\mathrm{a}}$. Mary Lilian Lourenço
}

Durante o desenvolvimento deste trabalho o autor recebeu auxílio financeiro do CNPq

São Paulo, janeiro de 2019 


\section{Propriedade de Bishop-Phelps-Bollobás}

Esta versão da dissertação contém as correções e alterações sugeridas pela Comissão Julgadora durante a defesa da versão original do trabalho, realizada em 21/02/2019. Uma cópia da versão original está disponível no

Instituto de Matemática e Estatística da Universidade de São Paulo.

Comissão Julgadora:

- Prof ${ }^{\mathrm{a}}$. Dra ${ }^{\mathrm{a}}$. Mary Lilian Lourenço (orientadora) - IME-USP

- Prof. Dr. Nilson da Costa Bernardes Junior - UFRJ

- Prof. Dr. Walter Alberto de Siqueira Pedra - IF-USP 


\section{Agradecimentos}

Destino meu primeiro e maior agradecimento a Deus, por sempre me acompanhar e tornar tudo possível.

Em segundo lugar, agradeço à minha mãe, que sempre me apoiou nos momentos de triunfo e de adversidade que acompanham a realização de um trabalho. Agradeço ao meu pai, por todas as conversas e conselhos. Fui abençoada com os pais mais compreensivos e maravilhosos que eu poderia desejar, e devo tudo que sou a eles.

Agradeço também à minha família, por sempre estar ao meu lado.

Agradeço à minha orientadora, Mary Lilian, por acreditar em mim e me dar a oportunidade de ser sua aluna. Levarei comigo não apenas seus ensinamentos de matemática, mas também aprendizados de esforço e dedicação.

Voltaire dizia que todas as riquezas do mundo não valem um bom amigo. Agradeço aos meus amigos, por tornarem a minha vida mais leve e sempre compreenderem o porquê de eu ter que trabalhar naquele feriado.

Agradeço aos meus professores do ensino fundamental e médio, por despertarem em mim o desejo de aprender, e aos da faculdade, que ao longo destes anos tanto me ajudaram a suprir este desejo.

Agradeço aos professores Nilson Bernardes e Walter Pedra, por aceitarem compor a banca examinadora e por suas sugestões e correções.

Por fim, agradeço ao CNPq pelo apoio financeiro. 


\section{Resumo}

FRAGA, J. T. Propriedade de Bishop-Phelps-Bollobás. 2018. 111 f. Dissertação (Mestrado) - Instituto de Matemática e Estatística, Universidade de São Paulo, São Paulo, 2018.

Este trabalho tem como objetivo principal estudar determinadas propriedades de pares de espaços de Banach de forma que satisfaçam a Propriedade de Bishop-Phelps-Bollobás para operadores (BPBp), acompanhando a evolução histórica do assunto. Inicialmente apresentamos demonstrações dos Teoremas de Bishop-Phelps e Bishop-Phelps-Bollobás, e em seguida passamos a estudar as versões destes resultados para operadores, entre as quais enfatizamos a segunda. Com esse objetivo, definimos a Propriedade de Bishop-Phelps-Bollobás para operadores, introduzida por Acosta et al. em [AAGM08], e apresentamos dois resultados deste artigo, que afirmam que se os espaços de Banach $X$ e $Y$ têm dimensão finita, então $(X, Y)$ satisfaz a BPBp, e que se o espaço de Banach $Y$ tem a propriedade $\beta$ de Lindenstrauss, então $(X, Y)$ satisfaz a BPBp para todo espaço de Banach $X$. Em seguida estudamos o artigo [AGKM17], que apresenta uma classe de espaços de Banach $Y$ tais que $\left(c_{0}, Y\right)$ satisfaz a BPBp, e mostra que embora nesta classe estejam contidos os espaços de Banach uniformemente convexos e aqueles que satisfazem a propriedade $\beta$, ela ainda contêm outros exemplos de espaços.

Palavras-chave: operadores que atingem a norma, propriedade de Bishop-Phelps-Bollobás, propriedade $\beta$ de Lindenstrauss. 


\section{Abstract}

FRAGA, J. T. Bishop-Phelps-Bollobás property. 2018. 120 f. Dissertação (Mestrado) - Instituto de Matemática e Estatística, Universidade de São Paulo, São Paulo, 2018.

The main purpose of this work is to study certain properties of pairs of Banach spaces in a way that satisfies the Bishop-Phelps-Bollobás property for operators (BPBp), following the historical evolution of the subject. Firstly we present proofs of the Bishop-Phelps and Bishop-Phelps-Bollobás theorems, and then proceed to study versions of these results for operators, of which we emphasize the second one. To this purpose, we define the Bishop-Phelps-Bollobás property for operators, introduced by Acosta et al. in [AAGM08], and present two results of this paper, which state that if $X$ and $Y$ are finite-dimensional Banach spaces, then $(X, Y)$ satisfies BPBp, and that if the Banach space $Y$ has the property $\beta$ of Lindenstrauss, then $(X, Y)$ satisfies BPBp for every Banach space $X$. Next we study paper [AGKM17], which presents a class of Banach spaces $Y$ such that $\left(c_{0}, Y\right)$ satisfies BPBp, and shows that although this class contains the uniformly rotund spaces and those satisfying property $\beta$, there are other examples of spaces in it.

Keywords: operators which attain their norm, Bishop-Phelps-Bollobás property, property $\beta$ of Lindenstrauss. 


\section{Sumário}

$\begin{array}{ll}\text { Lista de Símbolos } & \text { ix }\end{array}$

Introdução

1 Conceitos preliminares $\quad 1$

1.1 Resultados básicos . . . . . . . . . . . . . . . . . . . . 1

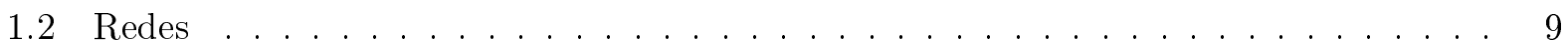

1.3 Topologias fraca e fraca-estrela . . . . . . . . . . . . . . . . . . . 10

1.4 Espaços estritamente convexos . . . . . . . . . . . . . . . . . . 12

1.5 Espaços uniformemente convexos . . . . . . . . . . . . . . . . . 15

1.6 Somabilidade em espaços normados . . . . . . . . . . . . . . . . . . 17

2 Funcionais que atingem a norma e seus teoremas clássicos $\quad 25$

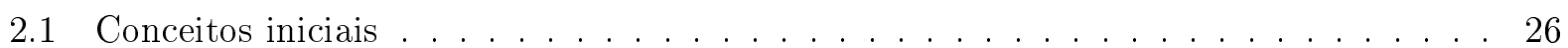

2.2 Teorema de Bishop-Phelps . . . . . . . . . . . . . . . . . . . 28

2.3 Teorema de Bishop-Phelps-Bollobás . . . . . . . . . . . . . . . . . . . . . 41

3 Operadores que atingem a norma $\quad 45$

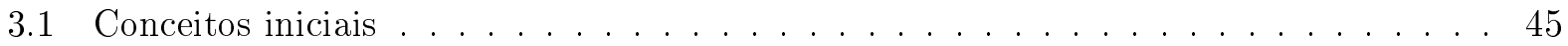

3.2 Bishop-Phelps para operadores . . . . . . . . . . . . . . . . 49

3.3 Propriedade de Bishop-Phelps-Bollobás para operadores . . . . . . . . . . . . . 52

Referências Bibliográficas $\quad 85$ 
viii SUMÁRIO 


\section{Lista de Símbolos}

$\mathbb{K} \quad$ Corpo dos números reais, $\mathbb{R}$, ou dos complexos, $\mathbb{C}$.

$\mathbb{R}_{\geq 0} \quad$ Conjunto dos números reais não-negativos.

$\operatorname{Re}(z) \quad$ Parte real do número complexo $z$.

$\operatorname{Im}(z) \quad$ Parte imaginária do número complexo $z$.

$A^{\complement} \quad$ Complementar do conjunto A relativo a um conjunto $U \supset A$, isto é, $U \backslash A$. O conjunto $U$ em geral ficará claro pelo contexto.

$X, Y \quad$ Espaços normados sobre o corpo $\mathbb{K}$.

$B_{X}(x ; r) \quad$ Bola aberta de centro $x \in X$ e raio $r \in \mathbb{R}, r>0$, em $X$. Quando o espaço $X$ for claro do contexto, ele será omitido da notação.

$B_{X} \quad$ Bola unitária e fechada em $X$.

$S_{X} \quad$ Esfera unitária em $X$.

$X^{*} \quad$ Dual topológico de $X$.

$\mathcal{L}(X, Y) \quad$ Espaço normado dos operadores lineares contínuos definidos entre $X$ e $Y$.

$\mathcal{K}(X, Y) \quad$ Espaço normado dos operadores lineares compactos definidos entre $X$ e $Y$.

$\mathcal{F}(X, Y) \quad$ Espaço normado dos operadores lineares contínuos de posto finito definidos entre $X$ e $Y$.

$\mathcal{N} \mathcal{A}_{X} \quad$ Conjunto $\left\{f \in X^{*}: f\right.$ atinge sua norma $\}$.

$\mathcal{N} \mathcal{A}(X, Y)$ Conjunto $\{T \in \mathcal{L}(X, Y): T$ atinge sua norma $\}$.

$\bar{A} \quad$ Fecho de um subconjunto $A \subset X$.

$A^{\circ} \quad$ Interior de um subconjunto $A \subset X$.

$\partial A \quad$ Fronteira de um subconjunto $A \subset X$.

$c_{0} \quad$ Espaço de Banach das sequências em $\mathbb{K}$ convergindo a 0 , munido da norma $\|.\|_{\infty}$.

$\ell_{p}^{n} \quad$ Espaço de Banach $\left(\mathbb{K}^{n},\|\cdot\|_{p}\right)$, para $n \in \mathbb{N}$ e $1 \leq p \leq \infty$.

$\ell_{p} \quad$ Espaço de Banach das sequências p-somáveis em $\mathbb{K}$, para $1 \leq p<\infty$, munido da norma $\|.\|_{p}$.

$\ell_{\infty} \quad$ Espaço de Banach das sequências limitadas em $\mathbb{K}$, munido da norma $\|.\|_{\infty}$.

$C(K) \quad$ Espaço de Banach das funções $f: K \rightarrow \mathbb{K}$ contínuas, munido da norma $\|f\|_{\infty} \doteq$ $\sup _{x \in K}|f(x)|$, quando $K$ é um espaço topológico compacto Hausdorff. 


\section{Introdução}

Dado um espaço de Banach $X$ sobre $\mathbb{R}$ ou $\mathbb{C}$, denotamos por $B_{X}$ a sua bola fechada e unitária e $S_{X}$ a sua esfera unitária. Dado $f \in X^{*}$, o dual topológico de $X$, dizemos que $f$ atinge sua norma se existe $x_{0} \in B_{X}$ tal que $\left|f\left(x_{0}\right)\right|=\|f\|$. Um fato simples de notar é que se $X$ é reflexivo, então todo elemento do dual atinge a sua norma. A recíproca deste resultado é o famoso Teorema de James, demonstrado por R. James primeiro para espaços de Banach separáveis [Jam57], em 1957, e depois para o caso geral, em 1964 [Jam64].

Na mesma época, inspirados pelo trabalho de James, R. Phelps e E. Bishop também começam a estudar funcionais que atingem a norma. Phelps percebeu que todo espaço de Banach clássico (isto é, os espaços da forma $C(K)$ ou $L_{p}(\mu)$, com $K$ um compacto Hausdorff e $\mu$ uma medida) tinha a seguinte propriedade: o conjunto dos funcionais lineares e contínuos que atingem a norma é denso no dual. Em vista dos resultados de James, parecia a ele que espaços com tal propriedade eram quase reflexivos, razão pela qual os chamou de subreflexivos [Phe57]. Porém, em 1961, junto com Bishop, viria a descobrir que todo espaço de Banach é subreflexivo, fato que ficou conhecido como Teorema de Bishop-Phelps [BP61].

Alguns anos mais tarde, em 1970, B. Bollobás provou uma "versão quantitativa"do Teorema de Bishop-Phelps, que ficou conhecida como Teorema de Bishop-Phelps-Bollobás [Bol70]. Mais precisamente, mostrou que para qualquer $0<\epsilon<1$, se $x \in S_{X}$ e $f \in S_{X^{*}}$ obedecem a $|1-f(x)|<\epsilon^{2} / 4$, é possível encontrar elementos $y \in S_{X}$ e $g \in S_{X^{*}}$ distando de menos de $\epsilon$ de $x$ e $f$, respectivamente, e tais que $g$ atinge sua norma em $y$.

É natural questionar se versões análogas destes resultados valem quando tratamos de operadores entre dois espaços de Banach $X$ e $Y$. Seja $\mathcal{L}(X, Y)$ o conjunto dos operadores lineares contínuos definidos entre $X$ e $Y$. Da mesma forma, um operador $T \in \mathcal{L}(X, Y)$ atinge sua norma se existe $x_{0} \in$ $B_{X}$ tal que $\left\|T\left(x_{0}\right)\right\|=\|T\|$. Denominamos $\mathcal{N} \mathcal{A}(X, Y) \doteq\{T \in \mathcal{L}(X, Y): T$ atinge sua norma $\}$.

Antes mesmo de Bollobás apresentar o Teorema de Bishop-Phelps-Bollobás, Lindenstrauss já havia obtido muitos resultados notáveis no sentido de obter generalizações do Teorema de BishopPhelps a operadores no seu artigo [Lin63], de 1963. Mostrou, por exemplo, que um Teorema do tipo Bishop-Phelps, isto é, $\mathcal{N} \mathcal{A}(X, Y)$ ser denso em $\mathcal{L}(X, Y)$, não vale para espaços de Banach $X$ e $Y$ arbitrários. Introduziu também neste artigo a propriedade $\beta$ e mostrou que se $Y$ tem tal propriedade, então $\mathcal{N} \mathcal{A}(X, Y)$ é denso em $\mathcal{L}(X, Y)$ para qualquer espaço de Banach $X$. Como exemplos de espaços satisfazendo a propriedade $\beta$, podemos citar $c_{0}, \ell_{\infty}$ e $\ell_{\infty}^{n}$, para todo $n \in \mathbb{N}$. Mostrou ainda que quando $X$ é reflexivo, $\mathcal{N} \mathcal{A}(X, Y)$ é denso em $\mathcal{L}(X, Y)$ para todo espaço de Banach $Y$.

A partir de então, surgiram inúmeros trabalhos neste tema. Por exemplo, em 1986, no seu artigo [Sch83], Schachermayer introduziu a propriedade $\alpha$, e mostrou que ela também é uma condição suficiente sobre espaços de Banach $X$ para que $\mathcal{N} \mathcal{A}(X, Y)$ seja denso em $\mathcal{L}(X, Y)$ para todo espaço de 
Banach $Y$. O espaço de Banach $\ell_{1}$ é um exemplo de espaço não-reflexivo satisfazendo a propriedade $\alpha$. Outro exemplo de trabalho nesse sentido é o artigo de Acosta e Aguirre de 1996, [AA96], que apresenta uma outra condição suficiente sobre espaços de Banach $Y$ para que $\mathcal{N} \mathcal{A}(X, Y)$ seja denso em $\mathcal{L}(X, Y)$ para qualquer espaço de Banach $X$.

O estudo de extensões do Teorema de Bishop-Phelps-Bollobás a operadores teve início em 2008, quando Acosta et al. introduziram a Propriedade de Bishop-Phelps-Bollobás para operadores (BPBp), em [AAGM08]. Diversos resultados a respeito desta propriedade foram feitos neste mesmo artigo. Foi mostrado, por exemplo, que se $X$ e $Y$ têm dimensão finita, o par $(X, Y)$ satisfaz a BPBp, e que se $Y$ tem a propriedade $\beta$ de Lindenstrauss, o par $(X, Y)$ satisfaz a BPBp para qualquer espaço de Banach $X$, generalizando um resultado de Lindenstrauss citado anteriormente. Além disso, foram caracterizados os espaços de Banach $Y$ para os quais $\left(\ell_{1}, Y\right)$ satisfaz a BPBp, e demonstrado que se $Y$ for uniformemente convexo, então $\left(\ell_{\infty}^{n}, Y\right)$ satisfaz a BPBp, para todo $n \in \mathbb{N}$.

Desde a introdução destes conceitos, o interesse nesse assunto cresceu e podemos atualmente encontrar diversos trabalhos sobre a BPBp. Por exemplo, Acosta et al. mostraram que os pares da forma $(C(K), C(S))$ satisfazem a BPBp para quaisquer espaços topológicos compactos Hausdorff $K$ e $S\left[\mathrm{ABGC}^{+} 14\right]$. Para o caso real, Kim, Lee e Lin provaram que o par $\left(L_{\infty}, Y\right)$ satisfaz a BPBp sempre que $Y$ for uniformemente convexo [KLL16]. Para o caso complexo, Acosta mostrou que o par $\left(C_{0}(L), Y\right)$ satisfaz a BPBp para todo espaço uniformemente convexo $Y$ e qualquer espaço topológico Hausdorff localmente compacto $L$ [Aco16]. Existe também uma caracterização de espaços de Banach $Y$ tais que o par $\left(\ell_{\infty}^{3}, Y\right)$ satisfaz a BPBp $\left[\mathrm{ABGG}^{+} 15\right]$, e uma para que $\left(\ell_{\infty}^{4}, Y\right)$ satisfaça a propriedade [ADSM19].

Entretanto, o caso do par $\left(c_{0}, Y\right)$ é diferente dos supracitados, e ainda não há uma caracterização dos pares $\left(c_{0}, Y\right)$ que satisfazem a BPBp. Em [AAGM08], após a demonstração de que $\left(\ell_{\infty}^{n}, Y\right)$ satisfaz a BPBp para todo espaço $Y$ uniformemente convexo, ficou aberta a seguinte pergunta: o par $\left(c_{0}, Y\right)$ também satisfaz a BPBp se $Y$ for uniformemente convexo? Esta pergunta viria a ser respondida de forma afirmativa em 2012, por Kim [Kim13]. Acosta et al., em 2017, generalizaram este resultado, apresentando uma classe maior de espaços de Banach $Y$ tais que o par $\left(c_{0}, Y\right)$ satisfaz a BPBp [AGKM17]. Os espaços uniformemente convexos e aqueles que satisfazem a propriedade $\beta$ de Lindenstrauss estão contidos nesta classe, mas é mostrado em [AGKM17] que ela também contêm outros espaços. Sendo assim, o resultado de Acosta et al. não só generaliza o trabalho de Kim em [Kim13], como também de fato fornece exemplos de pares $\left(c_{0}, Y\right)$ satisfazendo a BPBp que antes não eram conhecidos.

Como resumido na discussão acima, o tópico Propriedade de Bishop-Phelps-Bollobás tem grande importância para a Análise Funcional e é rico em publicações recentes, assim como em problemas abertos, motivos pelos quais foi escolhido para a dissertação de mestrado. O objetivo principal do trabalho é estudar determinadas propriedades de pares $(X, Y)$ de espaços de Banach de forma que satisfaçam a BPBp, acompanhando a evolução histórica do assunto. Ele será dividido em três capítulos.

No primeiro capítulo, enunciamos alguns resultados básicos de Análise Funcional e Topologia, necessários para o entendimento dos demais.

No segundo capítulo, apresentamos demonstrações dos Teoremas de Bishop-Phelps e BishopPhelps-Bollobás, feitas em [BP63] e [Bol70], respectivamente. Para demonstrar o primeiro, introdu- 
zimos os conceitos de cone e funcionais suporte, que permitem obter outros resultados interessantes, dentre os quais destacamos o Teorema de Bishop-Phelps para funcionais suporte, resultado que também é apresentado neste capítulo.

No terceiro capítulo, passamos a estudar as versões do Teorema de Bishop-Phelps e BishopPhelps-Bollobás para operadores. A primeira delas será discutida apenas de maneira breve, sendo assim daremos mais ênfase à segunda.

Com esse objetivo, introduzimos os conceitos de ponto exposto e fortemente exposto, assim como o de famílias fortemente e uniformemente expostas por uma função. Apresentamos também a propriedade $\beta$ de Lindenstrauss, e damos alguns exemplos de espaços que a satisfazem.

Estudamos em seguida algumas propriedades simples da extensão do Teorema de Bishop-Phelps a operadores e apresentamos a demonstração do resultado de [Lin63] que afirma que se $Y$ for estritamente convexo e existir um operador não-compacto definido entre $c_{0}$ e $Y$, então $\mathcal{N} \mathcal{A}\left(c_{0}, Y\right)$ não é denso em $\mathcal{L}\left(c_{0}, Y\right)$. A partir deste resultado, mostramos um exemplo concreto de espaço $Y$ para o qual $\mathcal{N} \mathcal{A}\left(c_{0}, Y\right)$ não é denso em $\mathcal{L}\left(c_{0}, Y\right)$.

Passamos então a estudar a extensão do Teorema de Bishop-Phelps-Bollobás a operadores. Para isso, definimos a Propriedade de Bishop-Phelps-Bollobás, de Acosta et al. [AAGM08]. Exibimos as demonstrações deste artigo de que o par $(X, Y)$ satisfaz a BPBp se $X$ e $Y$ têm dimensão finita e de que $(X, Y)$ satisfaz a BPBp para todo espaço de Banach $X$, se $Y$ tem a propriedade $\beta$.

Posteriormente, concentramos o estudo em pares de espaços de Banach da forma $\left(c_{0}, Y\right)$, baseandonos no artigo [AGKM17]. Apresentamos a classe de espaços de Banach $Y$ lá introduzida e a demonstração de que espaços contidos nesta classe são tais que $\left(c_{0}, Y\right)$ satisfaz a BPBp. Para o estudo desta demonstração, apresentamos alguns lemas auxiliares, alguns dos quais são feitos em [AAGM08], e outros em [AGKM17].

Por fim, apresentamos a demonstração feita em [AGKM17] de que, embora na classe de espaços de Banach introduzida neste artigo estejam contidos os espaços de Banach uniformemente convexos e aqueles que possuem a propriedade $\beta$, ainda há outros exemplos de espaços nesta classe. De fato, no último resultado deste artigo, o qual também apresentamos, é mostrado que para qualquer espaço de Banach uniformemente convexo $(Y,\|\|$.$) de dimensão maior que 1$ é possível definir uma norma |||.||| em $Y$ equivalente à original e arbitrariamente próxima desta, de forma que o espaço $(Y,|||\cdot|||)$ esteja na classe definida, mas não satisfaça a propriedade $\beta$ nem seja uniformemente convexo. Para finalizar, apresentamos o resultado de Kim que afirma que, no caso real, se for dado que $Y$ é estritamente convexo, é possível caracterizar os espaços de Banach $\left(c_{0}, Y\right)$ que satisfazem a BPBp [Kim13]. 


\section{Capítulo 1}

\section{Conceitos preliminares}

Neste capítulo, trataremos dos conceitos preliminares que serão necessários no decorrer do trabalho. Resultados usualmente tratados em cursos de graduação em matemática serão apenas enunciados. A menos que se diga o contrário, sempre que falarmos de espaços normados ao longo do trabalho, estaremos nos referindo a espaços sobre $\mathbb{K}$, em que $\mathbb{K}=\mathbb{R}$ ou $\mathbb{K}=\mathbb{C}$. Denotamos os espaços normados em geral por $X$ e $Y$, e a norma destes espaços por $\|$.$\| .$

A primeira seção tratará de resultados básicos de Análise Funcional e Topologia. A segunda, de conceitos gerais de redes, e a terceira de topologias fraca e fraca-estrela. As duas seções seguintes tratarão, respectivamente, de espaços estritamente convexos e uniformemente convexos. A última seção tratará do conceito de somabilidade em um espaço normado, e este será aplicado ao espaço de Banach $c_{0}$, usado com frequência no capítulo 3 .

Para a demonstração completa dos resultados apresentados neste capítulo, indicamos [Meg98] e [Wil04].

\subsection{Resultados básicos}

Definição 1.1.1. Seja $(P, \leq)$ um conjunto parcialmente ordenado. Dizemos que $\mathcal{C} \subset P$ é uma cadeia de $P$ se $\mathcal{C}$ for totalmente ordenado com a ordem induzida de $P$.

Teorema 1.1.2 (Lema de Zorn). Seja $(P, \leq)$ um conjunto parcialmente ordenado tal que $P \neq \emptyset$. Suponha que toda cadeia não-vazia de $P$ possua cota superior em $P$. Então $P$ tem um elemento maximal.

A seguir enunciamos resultados bem conhecidos de Álgebra Linear.

Lema 1.1.3. Sejam $V$ um espaço vetorial e $f, f_{1}, f_{2}, \ldots, f_{n}$ funcionais lineares em $V$ tais que $\bigcap_{i=1}^{n} \operatorname{Ker}\left(f_{i}\right) \subset \operatorname{Ker}(f)$. Então $f$ é combinação linear de $f_{1}, f_{2}, \ldots, f_{n}$.

Proposição 1.1.4. Seja $V$ um espaço vetorial com produto interno. Sejam $v_{1}, \ldots, v_{n} \in V$ vetores não-nulos, $n \geq 1$. Então $\left\|v_{1}+\ldots+v_{n}\right\|=\left\|v_{1}\right\|+\ldots+\left\|v_{n}\right\|$ se, e somente se, $v_{i}=k_{i} v_{1}$, com $k_{i}>0$, para todo $i=1, \ldots, n$.

Da Proposição acima segue facilmente o Corolário seguinte.

Corolário 1.1.5. Sejam $a_{1}, \ldots, a_{n}$ reais estritamente positivos e $\lambda_{1}, \ldots, \lambda_{n} \in S_{\mathbb{K}}$. Se $\lambda_{1} a_{1}+\ldots+$ $\lambda_{n} a_{n}=a_{1}+\ldots+a_{n}$, então $\lambda_{1}=\ldots=\lambda_{n}=1$. 
Se $A$ e $B$ são subconjuntos de um espaço vetorial $V$, definimos $A+B \doteq\{a+b \in V: a \in A$ e $b \in$ $B$ \}. Se o conjunto $B$ contém apenas um elemento $v$, denotaremos $A+B$ simplesmente por $A+v$. Se $\Lambda \subset \mathbb{K}$, definimos $\Lambda A \doteq\{\lambda a: \lambda \in \Lambda$ e $a \in A\}$. Se o conjunto $\Lambda$ contêm apenas um elemento $\lambda$, denotaremos $\Lambda A$ simplesmente por $\lambda A$.

Recordamos que um subconjunto $A$ de um espaço vetorial $V$ sobre $\mathbb{K}$ é dito equilibrado se $\lambda A \subset A$ sempre que $\lambda \in \mathbb{K}$ é tal que $|\lambda| \leq 1$.

Proposição 1.1.6. Sejam $X=(X,\|\|$.$) um espaço normado e B \subset X$ um subconjunto fechado, limitado, equilibrado, convexo e tal que $0 \in B^{\circ}$. Existe uma norma em $X$ que tem o conjunto $B$ como bola unitária fechada.

Demonstração. Para cada $x \in X$, defina $A_{x} \doteq\{r \geq 0: x \in r B\}$. Observemos que $A_{x}$ é não-vazio para todo $x \in X$, pois $0 \in B^{\circ}$. Seja

$$
\begin{aligned}
\|\| . \| \mid: X & \rightarrow \mathbb{R}_{\geq 0} \\
x & \mapsto \inf A_{x} .
\end{aligned}
$$

Mostraremos a seguir que $\|||\|||$ é uma norma para $X$. Temos que $0 \in A_{0}$, e portanto $\||0|\|=0$. Como $B$ é limitado, existe $M>0$ tal que $\|y\| \leq M$ para todo $y \in B$. Logo, dado $x \in X \backslash\{0\}$, se $0 \leq r<\frac{\|x\|}{M}$, então $x \notin r B$. Portanto,

$$
A_{x} \subset\left[\frac{\|x\|}{M}, \infty\right)
$$

o que implica que $\inf A_{x}=\|\| x\|\|>0$.

Se $x=y=0$, é claro que $\||x+y\||=0 \leq\|\| x\|\|+\|\mid\| y \|=0$. Considere $x, y \in X$ de forma que não sejam ambos nulos. Vamos mostrar que $A_{x}+A_{y} \subset A_{x+y}$. Tomemos $\lambda_{x} \in A_{x}, \lambda_{y} \in A_{y}$ arbitrários. Temos que $x=\lambda_{x} b_{x}$ e $y=\lambda_{y} b_{y}$, com $b_{x}, b_{y} \in B$. Como $B$ é convexo,

$$
x+y=\left(\lambda_{x}+\lambda_{y}\right)\left(\frac{\lambda_{x}}{\lambda_{x}+\lambda_{y}} b_{x}+\frac{\lambda_{y}}{\lambda_{x}+\lambda_{y}} b_{y}\right) \in\left(\lambda_{x}+\lambda_{y}\right) B,
$$

ou seja, $\lambda_{x}+\lambda_{y} \in A_{x+y}$, como queríamos demonstrar. Em particular,

$$
\inf \left(A_{x}+A_{y}\right)=\inf A_{x}+\inf A_{y}=\|\| x|\|+\|| y|\|\geq\| x+y \||=\inf A_{x+y} .
$$

Para finalizar, vamos mostrar que para todos $\lambda \in \mathbb{K}$ e $x \in X$, vale que $\||\lambda x|\|=|\lambda| \cdot|||x|||$. Para $\lambda=0$, isso é claro. Suponhamos que $\lambda \in S_{\mathbb{K}}$. Como $B$ é equilibrado, para todos $x \in X$ e $r \geq 0$ temos que

$$
\lambda x \in r B \Rightarrow x \in \frac{r}{\lambda} B=r(\bar{\lambda} B) \subset r B,
$$

e portanto $A_{\lambda x} \subset A_{x}$. Trocando $\lambda$ por $\bar{\lambda}$ e $x$ por $\lambda x$, um argumento análogo mostra que $A_{\bar{\lambda}(\lambda x)}=$ $A_{x} \subset A_{\lambda x}$, e então $A_{x}=A_{\lambda x}$ para todos $x \in X$ e $\lambda \in S_{\mathbb{K}}$. 
Suponhamos agora que $\lambda$ seja um real positivo. Para todos $r \geq 0$ e $x \in X$, vale que

$$
r \in A_{\lambda x} \Leftrightarrow \lambda x \in r B \Leftrightarrow x \in \frac{r}{\lambda} B \Leftrightarrow \frac{r}{\lambda} \in A_{x},
$$

e assim $A_{\lambda x}=\lambda A_{x}$ para todo $x \in X$ nesse caso. Finalmente, considere $\lambda \in \mathbb{K} \backslash\{0\}$ arbitrário. Podemos escrever $\lambda=|\lambda| \sigma$, com $\sigma \in S_{\mathbb{K}}$. Pelo que mostramos, $A_{\lambda x}=A_{|\lambda| \sigma x}=|\lambda| A_{\sigma x}=|\lambda| A_{x}$, e então

$$
\||\lambda x|\|=\inf A_{\lambda x}=\inf |\lambda| A_{x}=|\lambda| \inf A_{x}=|\lambda| \cdot|||x||| .
$$

Provamos assim que |||.||| é uma norma para $X$. Resta mostrar que $B$ é a bola unitária fechada do espaço normado $(X, \||| \cdot|| \mid)$, que denotaremos $B_{(X,|||.|||)}$.

Se $x \in B$, então $1 \in A_{x}$, o que implica $\left\||x \|| \leq 1\right.$ e assim $x \in B_{(X,|\|. \mid\|)}$. Por outro lado, suponha que $x \in B_{(X, \||.|||)}$, isto é, $\left\||x \|| \leq 1\right.$. Existe uma sequência $\left(r_{n}\right)_{n \in \mathbb{N}}$ de reais não-negativos tal que $r_{n} \rightarrow\|\| x \|$ e $\frac{x}{r_{n}} \in B$ para cada $n \in \mathbb{N}$. Como $B$ é fechado, concluímos que $\frac{x}{\|x\|} \in B$, e então $x \in\|\| x \| \mid B \subset B$, já que $B$ também é equilibrado. Portanto, $B=B_{(X,\|.|.|\|)}$.

Mesmo que um espaço normado $X$ não seja completo, ele pode ser tratado como um subconjunto denso de um espaço de Banach. É o que enunciamos em seguida.

Proposição 1.1.7. Seja $X$ um espaço normado. Existe um espaço de Banach $\tilde{X}$ e uma imersão isométrica $A: X \rightarrow \tilde{X}$, tal que $A(X)$ é denso em $\tilde{X}$. O espaço $\tilde{X}$ é o único com essas propriedades, a menos de isometrias.

Chamamos o espaço $\tilde{X}$ acima de completamento de $X$. Para todos os propósitos do nosso trabalho, a Proposição acima permite identificar $A(X) \operatorname{com} X$. Dizemos então que o espaço normado $X$ é subconjunto denso do espaço de Banach $\tilde{X}$.

Existe um resultado análogo ao enunciado acima quando consideramos espaços com produto interno:

Proposição 1.1.8. Se $X$ é um espaço com produto interno, existe um espaço de Hilbert $H$ e uma imersão isométrica $A: X \rightarrow H$ que preserva produtos internos tal que $A(X)$ é denso em $H$. $O$ espaço $H$ é o único com essas propriedades, a menos de isometrias.

O Lema abaixo mostra que o interior de um subconjunto convexo em um espaço normado satisfaz a uma propriedade um pouco mais forte que a convexidade.

Lema 1.1.9. Sejam $X$ um espaço normado e $C \subset X$ um subconjunto convexo de interior não-vazio. Se $x \in C^{\circ}$ e $y \in C$, então $t x+(1-t) y \in C^{\circ}$, para todo $t \in(0,1]$.

Demonstração. Se $t \neq 0$, a aplicação $m_{t}: X \ni x \mapsto t x \in X$ é um homeomorfismo. Logo, $t C^{\mathrm{o}}$ é um conjunto aberto se $t \in(0,1]$. Da mesma forma, para todo $x_{0} \in X$, a aplicação $s_{x_{0}}: X \ni x \mapsto$ $x+x_{0} \in X$ é um homeomorfismo. Então para cada $x_{0} \in X, x_{0}+t C^{\mathrm{o}}$ é um conjunto aberto, assim como $\bigcup_{x_{0} \in(1-t) C}\left(t C^{\mathrm{o}}+x_{0}\right)=t C^{\mathrm{o}}+(1-t) C$, qualquer que seja $t \in(0,1]$.

Como $C$ é convexo, vale que $t C^{\mathrm{o}}+(1-t) C \subset C$, o que implica $t C^{\mathrm{o}}+(1-t) C \subset C^{\mathrm{o}}$ para $t \in(0,1]$. 
Corolário 1.1.10. Sejam $X$ um espaço normado e $C \subset X$ subconjunto convexo de $X$ com interior não-vazio. Então $C^{\circ}$ é denso em $C$.

Demonstração. Tome $x \in C^{\mathrm{o}}$ qualquer, e seja dado $y \in C$. Pelo Lema 1.1.9, $u_{t} \doteq t x+(1-t) y \in C^{\mathrm{o}}$, para todo $t \in(0,1]$. Além disso, $\left\|u_{t}-y\right\|=\|t x-t y\|=|t| \cdot|| x-y \mid \|^{\mathrm{t} \rightarrow 0^{+}} \longrightarrow$ 0. Como $y \in C$ era arbitrário,

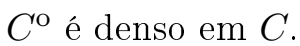

O Corolário abaixo será usado algumas vezes no decorrer do trabalho, razão pela qual está enunciado aqui.

Corolário 1.1.11. Sejam $X$ espaço normado, $Y \subset X$ um subconjunto denso e $C \subset X$ um subconjunto convexo, com $C^{\circ} \neq \emptyset$. Então $Y \cap C$ é denso em $C$.

Demonstração. Seja $\emptyset \neq U_{C} \subset C$ um subconjunto aberto de $C$. Então existe $\emptyset \neq U$ aberto de $X$ tal que $U_{C}=U \cap C$. Vamos mostrar que $U_{C} \cap(Y \cap C)=U_{C} \cap Y \neq \emptyset$. Observemos inicialmente que

$$
\left(C^{\mathrm{o}} \cap U_{C}\right) \cap Y \subset(C \cap U) \cap Y=U_{C} \cap Y .
$$

Como $Y$ é denso em $X$, basta mostrar que $\left(C^{\circ} \cap U\right)=\left(C^{\circ} \cap U_{C}\right) \neq \emptyset$. Mas isso segue diretamente do Corolário 1.1.10.

Como aplicação do Corolário anterior, temos a seguinte Proposição. Se $X$ é um espaço normado e $Y$ é um subespaço de $X$, denotamos a norma de elementos em $Y^{*}$ por $\|\cdot\|_{Y^{*}}$.

Proposição 1.1.12. Sejam $X$ um espaço normado e $Y \subset X$ subespaço denso de $X$. A aplicação

$$
\begin{aligned}
P: X^{*} & \rightarrow Y^{*} \\
f & \left.\mapsto f\right|_{Y}
\end{aligned}
$$

é uma isometria entre $X^{*}$ e $Y^{*}$.

Demonstração. Observemos inicialmente que de fato $P$ é linear, $\left.f\right|_{Y} \in Y^{*}$ e $|f|_{Y}(y) \mid \leq\|f\| \cdot\|y\|$, para todos $y \in Y, f \in X^{*}$. Então $\|P(f)\|_{Y^{*}} \leq\|f\|$, para todo $f \in X^{*}$.

Mostremos que $P$ é injetora. Seja $f \in X^{*}$ tal que $\left.f\right|_{Y}=0$. Para cada $x \in X$, existe uma sequência $\left(x_{n}\right)_{n \in \mathbb{N}}$ de elementos em $Y$ tal que $x_{n} \rightarrow x$. Logo, $\left.f\right|_{Y}\left(x_{n}\right)=0$ para cada $n \in \mathbb{N}$ e $\left.f\right|_{Y}\left(x_{n}\right)=f\left(x_{n}\right) \rightarrow f(x)$, o que implica $f(x)=0$. Portanto, $f=0$ e $P$ é injetora.

Agora verificaremos a sobrejetividade de $P$. Tome $g \in Y^{*}$. Dado $x \in X$, seja $\left(x_{n}\right)_{n \in \mathbb{N}}$ uma sequência em $Y$ tal que $x_{n} \rightarrow x$. Como $\left|g\left(x_{n}\right)-g\left(x_{m}\right)\right| \leq\|g\|_{Y^{*}} \cdot\left\|x_{n}-x_{m}\right\|$ para todos $m, n \in \mathbb{N}$ e a sequência $\left(x_{n}\right)_{n \in \mathbb{N}}$ é de Cauchy, $\left(g\left(x_{n}\right)\right)_{n \in \mathbb{N}}$ também é sequência de Cauchy em $\mathbb{K}$, e portanto converge. Além disso, se $\left(y_{n}\right)_{n \in \mathbb{N}}$ for outra sequência em $Y$ satisfazendo a $y_{n} \rightarrow x$, teremos

$$
\left|g\left(x_{n}\right)-g\left(y_{n}\right)\right| \leq\|g\|_{Y^{*}} \cdot\left\|x_{n}-y_{n}\right\| \rightarrow 0
$$

e então $\lim _{n \rightarrow \infty} g\left(x_{n}\right)=\lim _{n \rightarrow \infty} g\left(y_{n}\right)$.

Assim, podemos definir uma função $f: X \rightarrow \mathbb{K}$ por $f(x)=\lim _{n \rightarrow \infty} g\left(x_{n}\right)$, em que $\left(x_{n}\right)_{n \in \mathbb{N}}$ é uma sequência qualquer em $Y$ convergindo a $x$. É claro que $f$ é linear e $g=\left.f\right|_{Y}$. Resta verificar que $f \in X^{*}$, o que será feito em seguida. Dado $x \in X$, se $\left(x_{n}\right)_{n \in \mathbb{N}}$ for uma sequência em $Y$ convergindo a $x$, vale que 


$$
\left|g\left(x_{n}\right)\right| \leq\|g\|_{Y^{*}} \cdot\left\|x_{n}\right\| \text {, para todo } n \in \mathbb{N}
$$

e, portanto, fazendo $n \rightarrow \infty$, vem que $|f(x)| \leq\|g\|_{Y^{*}} \cdot\|x\|$. Logo, $f \in X^{*}$, e $P$ é sobrejetora.

Finalmente, mostremos que $P$ é isometria. Se $f_{0}=0 \in X^{*}$, é claro que $\left\|P\left(f_{0}\right)\right\|_{Y^{*}}=\left\|f_{0}\right\|=0$, e já vimos que $\|P(f)\|_{Y^{*}} \leq\|f\|$, para todo $f \in X^{*}$. Então resta mostrar que dado $f \neq 0 \in X^{*}$, $\|P(f)\|_{Y^{*}} \geq\|f\|$. Faremos isso a seguir.

Seja $f \neq 0 \in X^{*}$. Se $\epsilon>0$, existe $x_{0} \in B_{X}$ tal que

$$
\|f\|-\frac{\epsilon}{2}<\left|f\left(x_{0}\right)\right|
$$

Além disso, pelo Corolário 1.1.11, $B_{X} \cap Y$ é denso em $B_{X}$, então existe $y_{0} \in B_{X} \cap Y$ satisfazendo a $\left\|x_{0}-y_{0}\right\|<\frac{\epsilon}{2\|f\|}$. Assim,

$$
\left|f\left(x_{0}\right)-f\left(y_{0}\right)\right| \leq\|f\| \cdot\left\|x_{0}-y_{0}\right\|<\frac{\epsilon}{2}
$$

e então por (1.1.1) e (1.1.2),

$$
\begin{aligned}
\left|f\left(y_{0}\right)\right| & \geq\left|f\left(x_{0}\right)\right|-\left|f\left(x_{0}\right)-f\left(y_{0}\right)\right| \\
& >\|f\|-\frac{\epsilon}{2}-\frac{\epsilon}{2} \\
& =\|f\|-\epsilon .
\end{aligned}
$$

Portanto, para todo $\epsilon>0$, existe $y_{0} \in B_{Y}=B_{X} \cap Y$ tal que $\left|f\left(y_{0}\right)\right|=\left|P(f)\left(y_{0}\right)\right|>|| f||-\epsilon$, o que garante $\|P(f)\|_{Y^{*}} \geq\|f\|$, e encerra a demonstração.

Não é difícil ver que a interseção arbitrária de subconjuntos convexos de um espaço normado $X$ é também um conjunto convexo. Dado um subconjunto $C \subset X$, a afirmação anterior garante a existência do menor subconjunto convexo de $X$ contendo $C$, isto é, um conjunto convexo contendo $C$ tal que qualquer subconjunto convexo de $X$ contendo $C$ deve também contê-lo. De fato, bastaria considerar a interseção de todos os subconjuntos convexos contendo $C$. Chamamos tal conjunto de envoltória convexa de $C$, e o denotamos $\operatorname{Env}(C)$. A seguir é enunciado um resultado a este respeito.

Proposição 1.1.13. Sejam $X, Y$ espaços normados, $C \subset X$ e $T: X \rightarrow Y$ transformação linear. $\operatorname{Entâo~} \operatorname{Env}(T(C))=T(\operatorname{Env}(C))$.

No decorrer do trabalho, para cada $n \in \mathbb{N}$ denotaremos por $e_{n}$ a sequência satisfazendo a $\left(e_{n}\right)_{i}=$ $\delta_{n i}$, isto é, a sequência cuja i-ésima coordenada é $\delta_{n i}$, para todo $i \in \mathbb{N}$. Em geral consideraremos essas sequências dentro de espaços normados, como $c_{0}$ e $\ell_{p}$.

Identificamos no resultado seguinte o espaço de Banach $c_{0}^{*}$.

Proposição 1.1.14. Os espaços $c_{0}^{*}$ e $\ell_{1}$ são isometricamente isomorfos por meio da isometria $c_{0}^{*} \ni f \mapsto\left(f\left(e_{n}\right)\right)_{n \in \mathbb{N}} \in \ell_{1}$.

A seguir enunciamos o Teorema de Hahn-Banach para espaços normados, um dos principais resultados de análise funcional, com dois de seus corolários principais. 
Teorema 1.1.15 (Hahn-Banach). Sejam $X$ um espaço normado e $Y \subset X$ um subespaço de $X$. Dado $f \in Y^{*}$, existe $\tilde{f} \in X^{*}$ tal que $\|\tilde{f}\|=\|f\|_{Y^{*}}$ e $f=\left.\tilde{f}\right|_{Y}$.

Corolário 1.1.16. Seja $X$ um espaço normado. Para todo $x \in X \backslash\{0\}$, existe $f \in S_{X^{*}}$ tal que $f(x)=\|x\|$.

Corolário 1.1.17. Sejam $X$ um espaço normado e $x, y \in X$ elementos distintos. Então existe $f \in X^{*}$ tal que $f(x) \neq f(y)$. Em outras palavras, $X^{*}$ separa pontos de $X$.

Dados dois espaços normados $X, Y$ e um operador $T \in \mathcal{L}(X, Y)$, para cada $f \in Y^{*}$ podemos definir a aplicação $(f \circ T): X \rightarrow \mathbb{K}$. É claro que $(f \circ T)$ é linear, e vale que $|(f \circ T)(x)| \leq\|f\| \cdot\|T\| \cdot\|x\|$. Então $(f \circ T) \in X^{*}$, tornando possível a seguinte definição:

Definição 1.1.18. Sejam $X, Y$ espaços normados e $T \in \mathcal{L}(X, Y)$. Definimos o operador transposto a $T$, denotado $T^{t}$, por

$$
\begin{aligned}
T^{t}: Y^{*} & \rightarrow X^{*} \\
f & \mapsto(f \circ T) .
\end{aligned}
$$

A Proposição abaixo será útil em alguns de nossos propósitos futuros.

Proposição 1.1.19. Sejam $X, Y$ espaços normados e $T \in \mathcal{L}(X, Y)$. Suponha que $G \subset S_{Y^{*}}$ seja tal que para todo $y \in Y,\|y\|=\sup _{f \in G}|f(y)|$. Então

$$
\left\|T^{t}\right\|=\sup _{f \in G}\left\|T^{t}(f)\right\|
$$

Demonstração. Se $f \in G$, temos que $\left\|T^{t}(f)\right\|=\sup \left\{|(f \circ T)(x)|: x \in S_{X}\right\}$. Então

$$
\begin{aligned}
\sup _{f \in G}\left\|T^{t}(f)\right\| & =\sup _{f \in G}\left(\sup _{x \in S_{X}}|(f \circ T)(x)|\right) \\
& =\sup _{x \in S_{X}}\left(\sup _{f \in G}|(f \circ T)(x)|\right) \\
& =\sup _{x \in S_{X}}\|T(x)\| \\
& =\|T\| .
\end{aligned}
$$

O Corolário 1.1.16 garante que $\|y\|=\sup _{f \in S_{Y^{*}}}|f(y)|$ para todo $y \in Y$. Assim, este é um caso particular do que foi mostrado acima, donde concluímos que

$$
\left\|T^{t}\right\|=\sup _{f \in S_{Y^{*}}}\left\|T^{t}(f)\right\|=\|T\|,
$$

e, portanto, $\left\|T^{t}\right\|=\sup _{f \in G}\left\|T^{t}(f)\right\|$.

Observemos que na demonstração da Proposição acima mostramos em particular que para todo $T \in \mathcal{L}(X, Y)$, vale que $\|T\|=\left\|T^{t}\right\|$. 
Se $X$ é um espaço normado, definimos a aplicação $C_{X}: X \rightarrow X^{* *}$, que satisfaz, para cada $x \in X$,

$$
\begin{aligned}
C_{X}(x): X^{*} & \rightarrow \mathbb{K} \\
f & \mapsto f(x) .
\end{aligned}
$$

A função $C_{X}$ é conhecida como injeção canônica de $X$ no seu bidual, $X^{* *}$. Vale que:

Proposição 1.1.20. A injeção canônica em um espaço normado é uma imersão isométrica.

Embora seja uma imersão isométrica, nem sempre a injeção canônica é sobrejetora. Quando isso acontece, o espaço recebe um nome especial.

Definição 1.1.21. Um espaço normado é dito reflexivo se a sua injeção canônica é sobrejetora.

O Teorema seguinte é um importante resultado de separação conhecido como Primeira Forma Geométrica do Teorema de Hahn-Banach.

Teorema 1.1.22 (Primeira Forma Geométrica do Teorema de Hahn-Banach). Sejam $X$ um espaço normado e $A, B \subset X$ subconjuntos convexos não-vazios de $X$ tais que $A$ é aberto $e$ $A \cap B=\emptyset$. Então existem $f \in X^{*} \backslash\{0\}$ e $a \in \mathbb{R}$ tais que

$$
\operatorname{Re} f(x)<a \leq \operatorname{Re} f(y) \text { para todos } x \in A \text { e } y \in B \text {. }
$$

O funcional $f \in X^{*} \backslash\{0\}$ dado acima pode ser escolhido de norma 1, ou satisfazendo a desigualdades opostas, como observamos abaixo.

Observação 1.1.23. (a) Nas condições do Teorema acima, se $g \doteq \frac{f}{\|f\|} \in S_{X^{*}}$, obtemos $\operatorname{Re} g(x)<$ $\frac{a}{\|f\|} \leq \operatorname{Re} g(y)$ para todos $x \in A$ e $y \in B$

(b) Nas condições do Teorema acima, se $g \doteq-f \in X^{*} \backslash\{0\}$, obtemos $\operatorname{Re} g(y) \leq-a<\operatorname{Re} g(x)$ para todos $x \in A$ e $y \in B$.

O resultado de separação que mais usaremos no trabalho é consequência simples da Primeira Forma Geométrica do Teorema de Hahn-Banach, e está demonstrado abaixo.

Teorema 1.1.24 (Teorema de separação de Eidelheit). Sejam $X$ espaço normado e $C_{1}, C_{2}$ subconjuntos convexos não-vazios de $X$ tais que $C_{2}^{\mathrm{o}} \neq \emptyset$ e $C_{1} \cap C_{2}^{\mathrm{o}}=\emptyset$. Então existem $f \in X^{*} \backslash\{0\}$ e $a \in \mathbb{R}$ satisfazendo a:

(i) $\operatorname{Re} f(x) \leq a$, para todo $x \in C_{2}$;

(ii) $\operatorname{Re} f(x)<a$, para todo $x \in C_{2}^{\mathrm{o}}$;

(iii) $\operatorname{Re} f(x) \geq a$, para todo $x \in C_{1}$.

Demonstração. Pelo Lema 1.1.9, $C_{2}^{\mathrm{o}}$ é convexo. Podemos, portanto, aplicar a Primeira Forma Geométrica do Teorema de Hahn-Banach para os conjuntos $C_{2}^{\text {o }}$ e $C_{1}$ para obter $f \in X^{*} \backslash\{0\}$ e $a \in \mathbb{R}$ satisfazendo a

$$
\operatorname{Re} f(x)<a \leq \operatorname{Re} f(y), \text { para todos } x \in C_{2}^{\mathrm{o}} \text { e } y \in C_{1} \text {. }
$$


Resta então mostrar que $\operatorname{Re} f(x) \leq a$ se $x \in C_{2}$. Tome $y \in C_{2}^{\mathrm{o}}$ qualquer. Novamente pelo Lema 1.1.9, para todos $t \in(0,1]$ e $x \in C_{2}$ vale que $(1-t) x+t y \in C_{2}^{\mathrm{o}}$. Então

$$
\operatorname{Re} f((1-t) x+t y)=(1-t) \operatorname{Re} f(x)+t \operatorname{Re} f(y)<a, \text { para todo } t \in(0,1] .
$$

Se $t \rightarrow 0^{+}$, vem que $\operatorname{Re} f(x) \leq a$, como queríamos mostrar.

Pelo mesmo motivo explicado na Observação 1.1.23, o funcional $f \in X^{*} \backslash\{0\}$ do Teorema acima pode ser tomado de norma 1 , ou satisfazendo desigualdes opostas às de (i), (ii) e (iii).

Seja agora $H$ um espaço de Hilbert, e dado $v \in H$, definamos o funcional linear $f_{v}$ por

$$
\begin{aligned}
f_{v}: H & \rightarrow \mathbb{K} \\
x & \mapsto<x, v>.
\end{aligned}
$$

Da desigualdade de Cauchy-Schwarz, sabemos que $f_{v} \in H^{*}$ e $\left\|f_{v}\right\| \leq\|v\|$. Vamos verificar que na verdade vale a igualdade. Se $v=0$, claramente $f_{v}=0$ e $\left\|f_{v}\right\|=\|v\|=0$. Se $v \neq 0, f_{v}\left(\frac{v}{\|v\|}\right)=$ $\frac{\|v\|^{2}}{\|v\|}=\|v\|$. Portanto, $\left\|f_{v}\right\|=\|v\|$ também nesse caso.

O Teorema seguinte é um resultado importante da teoria de espaços de Hilbert, que garante que todo funcional em $H^{*}$ é da forma $f_{v}$ para algum $v \in H$.

Teorema 1.1.25 (Riesz-Fréchet). Sejam $H$ um espaço de Hilbert e $f \in H^{*}$. Então existe um único $v_{0} \in H$ tal que

$$
f(x)=<x, v_{0}>\text { para todo } x \in H .
$$

Além disso, vale que $\|f\|=\left\|v_{0}\right\|$.

Para finalizar a seção, vamos definir os operadores compactos entre dois espaços normados e enunciar alguns resultados básicos sobre o assunto.

Definição 1.1.26. Sejam $X$ e $Y$ espaços normados. Um operador linear $T: X \rightarrow Y$ é dito compacto se $\overline{T\left(B_{X}\right)}$ é compacto.

Não é difícil de mostrar que o conjunto dos operadores lineares compactos definidos entre $X$ e $Y$ é subespaço vetorial de $\mathcal{L}(X, Y)$. Denotamos este subespaço por $\mathcal{K}(X, Y)$.

A seguir é enunciada uma caracterização simples de operadores compactos.

Proposição 1.1.27. Sejam $X$ e $Y$ espaços normados. O operador linear $T: X \rightarrow Y$ é compacto se, e somente se, para toda sequência limitada $\left(x_{n}\right)_{n \in \mathbb{N}}$ em $X$, a sequência $\left(T\left(x_{n}\right)\right)_{n \in \mathbb{N}}$ tem subsequência convergente em $Y$.

O último resultado relaciona os operadores lineares contínuos de posto finito com os operadores compactos. Dados os espaços normados $X$ e $Y$, denotamos por $\mathcal{F}(X, Y)$ o espaço normado dos operadores $T \in \mathcal{L}(X, Y)$ de posto finito.

Proposição 1.1.28. Se $X$ é espaço normado e $Y$ é espaço de Banach, então $\overline{\mathcal{F}(X, Y)} \subset \mathcal{K}(X, Y)$. 


\subsection{Redes}

É bem conhecido que sequências caracterizam a topologia de um espaço métrico. Porém, quando tratamos de espaços não-metrizáveis, pode não existir um sistema fundamental de vizinhanças enumerável para um dado ponto do espaço, o que impossibilita tal caracterização. Nesses casos, podemos utilizar o conceito de redes, que permite caracterizações similares.

Para mais detalhes sobre redes e a demonstração completa dos resultados enunciados nesta seção, indicamos [Wil04].

Definição 1.2.1. Um conjunto $\Lambda$ é dito dirigido se existe uma relação $\leq$ em $\Lambda$ satisfazendo a:

(i) $\lambda \leq \lambda$, para todo $\lambda \in \Lambda$;

(ii) se $\lambda_{1} \leq \lambda_{2}$ e $\lambda_{2} \leq \lambda_{3}$, então $\lambda_{1} \leq \lambda_{3}$, para todos $\lambda_{1}, \lambda_{2}, \lambda_{3} \in \Lambda$;

(iii) dados $\lambda_{1}, \lambda_{2} \in \Lambda$, existe $\lambda_{3} \in \Lambda$ tal que $\lambda_{1} \leq \lambda_{3}$ e $\lambda_{2} \leq \lambda_{3}$.

Definição 1.2.2. Uma rede em um conjunto $X$ é uma função $P: \Lambda \rightarrow X$, em que $\Lambda$ é um conjunto dirigido.

Observação 1.2.3. Nas condições da definição anterior, o ponto $P(\lambda)$, para dado $\lambda \in \Lambda$, é frequentemente denotado $x_{\lambda}$ e a rede $P$ é denotada $\left(x_{\lambda}\right)_{\lambda \in \Lambda}$.

Definição 1.2.4. Sejam $X$ um conjunto qualquer, $\Lambda$ um conjunto dirigido por uma relação $\leq, M$ um conjunto dirigido por uma relação $\preceq$ e $P: \Lambda \rightarrow X$ uma rede em $X$. Uma subrede de $P$ é uma composição $(P \circ \phi)$, em que $\phi: M \rightarrow \Lambda$ é uma função satisfazendo a:

(i) $\phi\left(\mu_{1}\right) \leq \phi\left(\mu_{2}\right)$ para todos $\mu_{1}, \mu_{2} \in M$ com $\mu_{1} \preceq \mu_{2}$, isto é, $\phi$ é crescente;

(ii) para cada $\lambda \in \Lambda$, existe $\mu \in M$ tal que $\lambda \leq \phi(\mu)$, isto é, $\phi$ é cofinal.

Observação 1.2.5. Nas condições da definição anterior, dado $\mu \in M$, o ponto $P(\phi(\mu))$ é frequentemente denotado $x_{\lambda_{\mu}}$ e a subrede $P \circ \phi$ é denotada $\left(x_{\lambda_{\mu}}\right)_{\mu \in M}$.

Definição 1.2.6. Sejam $X$ um espaço topológico e $\left(x_{\lambda}\right)_{\lambda \in \Lambda}$ uma rede em $X$. Dizemos que a rede $\left(x_{\lambda}\right)_{\lambda \in \Lambda}$ converge a $x \in X$ (escrevemos $x_{\lambda} \rightarrow x$ ) se, para toda vizinhança $U$ de $x$, existe $\lambda_{0} \in \Lambda$ tal que $x_{\lambda} \in U$ para todo $\lambda \in \Lambda$ satisfazendo a $\lambda \geq \lambda_{0}$.

No decorrer deste capítulo e dos próximos, se $X$ for um espaço topológico munido de uma topologia $\tau$ e a rede $\left(x_{\lambda}\right)_{\lambda \in \Lambda}$ convergir a $x \in X$, quando quisermos ressaltar que a convergência se dá na topologia $\tau$, diremos que $x_{\lambda} \stackrel{\tau}{\rightarrow} x$. Caso contrário, usaremos $x_{\lambda} \rightarrow x$ ou $\lim x_{\lambda}=x$.

A seguir enunciaremos a caracterização dos fechos de subconjuntos de espaços topológicos via redes, e, depois disso, dos compactos.

Proposição 1.2.7. Sejam $X$ um espaço topológico e $Y \subset X$. Então $x \in \bar{Y}$ se, e somente se, existe uma rede $\left(x_{\lambda}\right)_{\lambda \in \Lambda}$ em $Y$ tal que $x_{\lambda} \rightarrow x$.

Quando quisermos destacar que o fecho de um subespaço $Y$ de um espaço topológico $(X, \tau)$ é em relação à topologia $\tau$, escreveremos $\bar{Y}^{\tau}$ em vez de apenas $\bar{Y}$.

Proposição 1.2.8. Um espaço topológico $X$ é compacto se, e somente se, toda rede em $X$ tem subrede convergente. 
Funções contínuas definidas entre dois espaços topológicos também podem ser caracterizadas usando redes:

Proposição 1.2.9. Sejam $X$ e $Y$ espaços topológicos e $f: X \rightarrow Y$ uma função. A função $f$ é contínua no ponto $x_{0} \in X$ se, e somente se, $f\left(x_{\lambda}\right) \rightarrow f\left(x_{0}\right)$ para toda rede $\left(x_{\lambda}\right)_{\lambda \in \Lambda}$ em $X$ convergindo a $x_{0}$.

Se o espaço topológico $X$ for também um espaço vetorial e as operações de soma e produto por escalar forem contínuas, como é verdade nos espaços normados, a proposição acima implica o seguinte resultado.

Proposição 1.2.10. Sejam $X$ um espaço normado, $\alpha \in \mathbb{K} e\left(x_{\lambda}\right)_{\lambda \in \Lambda},\left(y_{\lambda}\right)_{\lambda \in \Lambda}$ redes em $X$ convergindo a $x \in X$ e $y \in X$, respectivamente. Então:

(i) $x_{\lambda}+y_{\lambda} \rightarrow x+y$;

(ii) $\alpha x_{\lambda} \rightarrow \alpha x$.

\subsection{Topologias fraca e fraca-estrela}

Muitas vezes é interessante considerar outras topologias em um dado espaço normado $X$ e no seu dual $X^{*}$. Nesta seção veremos os dois exemplos mais utilizados, a topologia fraca em $X$ e a fraca-estrela em $X^{*}$. Indicamos a referência [BPT15] para uma discussão detalhada do tema.

Para começar a tratar do assunto, definiremos a topologia gerada por uma família de funções em um conjunto $X$, e veremos algumas de suas propriedades principais. Sejam $X$ um conjunto, $\left(Y_{i}\right)_{i \in I}$ uma família de espaços topológicos e $\left(f_{i}\right)_{i \in I}$ uma família de funções $f_{i}: X \rightarrow Y_{i}$, para cada $i \in I$. Definimos

$$
\Phi \doteq\left\{f_{i}^{-1}(U): i \in I \text { e } U \subset Y_{i} \text { é aberto em } Y_{i}\right\}
$$

e

Definição 1.3.1. A topologia gerada pela famılia de funções $\left(f_{i}\right)_{i \in I}$ é a menor topologia em $X$ que contêm $\Phi$.

Observação 1.3.2. Para evitar confusão, vamos ressaltar aqui que uma topologia $\tau$ em um conjunto $X$ é dita a menor topologia contendo um determinado subconjunto $\Phi$ das partes de $X$ se for a interseção de todas as topologias de $X$ que contêm tal subconjunto. É fácil ver que, nesse caso, qualquer topologia em $X$ contendo $\Phi$ conterá $\tau$, e é nesse sentido que dizemos que ela é a menor topologia que contêm $\Phi$.

Notemos que a topologia gerada pela família $\left(f_{i}\right)_{i \in I}$ também é a menor topologia que torna todas as $f_{i}$ contínuas. Isto é, se $\tau^{\prime}$ for uma topologia em $X$ para a qual todas as $f_{i}$ são contínuas, $\tau^{\prime}$ contêm a topologia gerada pela família $\left(f_{i}\right)_{i \in I}$.

Utilizando ainda a notação dada acima, definamos

$$
\mathcal{B} \doteq\left\{\bigcap_{j=1}^{n} f_{i_{j}}^{-1}\left(U_{j}\right): n \in \mathbb{N}, i_{j} \in I \text { e } U_{j} \text { é aberto de } Y_{i_{j}}, \text { para } 1 \leq j \leq n\right\} .
$$

Proposição 1.3.3. O conjunto $\mathcal{B}$ é uma base para a topologia em $X$ gerada pela familia $\left(f_{i}\right)_{i \in I}$. 
A Proposição seguinte resume as principais propriedades da topologia definida acima.

Proposição 1.3.4. Sejam $X$ um conjunto, $\left(Y_{i}\right)_{i \in I}$ uma família de espaços topológicos, $\left(f_{i}\right)_{i \in I}$ uma família de funçôes $f_{i}: X \rightarrow Y_{i}$, para cada $i \in I$, e $\tau$ a topologia em $X$ gerada pelas $\left(f_{i}\right)_{i \in I}$. Então:

(a) Para cada $x \in X$, o conjunto

$\mathcal{B}_{x} \doteq\left\{\bigcap_{j=1}^{n} f_{i_{j}}^{-1}\left(U_{j}\right): n \in \mathbb{N}, i_{j} \in I\right.$ e $U_{j}$ é vizinhança aberta de $f_{i_{j}}(x)$ em $Y_{i_{j}}$, para $\left.1 \leq j \leq n\right\}$ constitui um sistema fundamental de vizinhanças para $x$ na topologia $\tau$.

(b) Seja $\left(x_{\lambda}\right)_{\lambda \in \Lambda}$ uma rede em $X$. Então $x_{\lambda} \stackrel{\tau}{\rightarrow} x \in X$ se, e somente se, $f_{i}\left(x_{\lambda}\right) \rightarrow f_{i}(x)$ para todo $i \in I$.

(c) Suponha que todos os $Y_{i}$ sejam espaços de Hausdorff. A topologia $\tau$ é de Hausdorff se, e somente se, a familia $\left(f_{i}\right)_{i \in I}$ separa pontos de $X$.

Agora definiremos a topologia fraca em um espaço normado $X$.

Definição 1.3.5. Se $X$ é um espaço normado, a topologia fraca em $X$, denotada $\sigma\left(X, X^{*}\right)$ (ou $w$ ), é a topologia gerada pelos funcionais em $X^{*}$.

Se $x \in X, n \in \mathbb{N}, \phi_{1}, \phi_{2}, \ldots, \phi_{n} \in X^{*}$ e $\epsilon>0$, definamos o conjunto

$$
V\left(x ; \phi_{1}, \phi_{2}, \ldots, \phi_{n} ; \epsilon\right) \doteq\left\{y \in X:\left|\phi_{j}(x)-\phi_{j}(y)\right|<\epsilon \text { para } 1 \leq j \leq n\right\}=\bigcap_{j=1}^{n} \phi_{j}^{-1}\left(B\left(\phi_{j}(x) ; \epsilon\right)\right) .
$$

A Proposição seguinte é apenas a reformulação da Proposição 1.3.4 e da construção geral feita acima para este caso particular.

Proposição 1.3.6. Seja $X$ um espaço normado. Então:

(a) Para todo $\phi \in X^{*}$, vale que $\phi:\left(X, \sigma\left(X, X^{*}\right)\right) \rightarrow \mathbb{K}$ é continua.

(b) $\sigma\left(X, X^{*}\right)$ está contida na topologia da norma.

(c) O conjunto

$$
\left\{V\left(x ; \phi_{1}, \phi_{2}, \ldots, \phi_{n} ; \epsilon\right): n \in \mathbb{N}, x \in X, \epsilon>0 \text { e } \phi_{j} \in X^{*} \text { para } 1 \leq j \leq n\right\}
$$

constitui uma base de abertos para $\sigma\left(X, X^{*}\right)$.

(d) Dado $x \in X$, conjunto

$$
\left\{V\left(x ; \phi_{1}, \phi_{2}, \ldots, \phi_{n} ; \epsilon\right): n \in \mathbb{N}, \epsilon>0 \text { e } \phi_{j} \in X^{*} \text { para } 1 \leq j \leq n\right\}
$$

constitui um sistema fundamental de vizinhanças abertas de $x$ na topologia $\sigma\left(X, X^{*}\right)$.

(e) Seja $\left(x_{\lambda}\right)_{\lambda \in \Lambda}$ uma rede em $X$. Então $x_{\lambda} \stackrel{w}{\rightarrow} x \in X$ se, e somente se, $\phi\left(x_{\lambda}\right) \rightarrow \phi(x)$ para todo $\phi \in X^{*}$. 
(f) A topologia fraca é de Hausdorff.

Passaremos agora a tratar a topologia fraca-estrela no dual de um espaço normado $X$. Lembremos que $C_{X}: X \rightarrow X^{* *}$ denota a injeção canônica no bidual de $X$.

Definição 1.3.7. Se $X$ é um espaço normado, a topologia fraca-estrela em $X^{*}$, denotada $\sigma\left(X^{*}, X\right)$ (ou $w^{*}$ ), é a topologia gerada pelos funcionais em $C_{X}(X)$.

Da mesma forma que fizemos anteriormente, dados $\phi \in X^{*}, n \in \mathbb{N}, x_{1}, x_{2}, \ldots, x_{n} \in X$ e $\epsilon>0$, tendo em vista que $C_{X}\left(x_{i}\right)(\psi)=\psi\left(x_{i}\right)$ para todos $\psi \in X^{*}$ e $i=1, \ldots, n$, definamos o conjunto

$$
W\left(\phi ; x_{1}, x_{2}, \ldots, x_{n} ; \epsilon\right) \doteq\left\{\psi \in X^{*}:\left|\phi\left(x_{i}\right)-\psi\left(x_{i}\right)\right|<\epsilon \text { para } 1 \leq j \leq n\right\} .
$$

Pela Proposição 1.3.4 e a discussão que a precede, teremos:

Proposição 1.3.8. Seja $X$ um espaço normado. Então:

(a) $\sigma\left(X^{*}, X\right)$ está contida na topologia $\sigma\left(X^{*}, X^{* *}\right)$.

(b) O conjunto

$$
\left\{W\left(\phi ; x_{1}, x_{2}, \ldots, x_{n} ; \epsilon\right): n \in \mathbb{N}, \phi \in X^{*}, \epsilon>0 \text { e } x_{j} \in X \text { para } 1 \leq j \leq n\right\}
$$

constitui uma base de abertos para $\sigma\left(X^{*}, X\right)$.

(c) Dado $\phi \in X^{*}$, conjunto

$$
\left\{W\left(\phi ; x_{1}, x_{2}, \ldots, x_{n} ; \epsilon\right): n \in \mathbb{N}, \epsilon>0 \text { e } x_{j} \in X \text { para } 1 \leq j \leq n\right\}
$$

constitui um sistema fundamental de vizinhanças abertas para $\phi$ na topologia $\sigma\left(X^{*}, X\right)$.

(d) Seja $\left(\phi_{\lambda}\right)_{\lambda \in \Lambda}$ uma rede em $X^{*}$. Então $\phi_{\lambda} \stackrel{w^{*}}{\rightarrow} \phi \in X^{*}$ se, e somente se, $\phi_{\lambda}(x) \rightarrow \phi(x)$ para todo $x \in X$.

(e) A topologia fraca-estrela é de Hausdorff.

Para finalizar esta seção, enunciamos um importante Teorema a respeito da topologia fracaestrela.

Teorema 1.3.9 (Banach-Alaoglu). Para todo espaço normado $X, B_{X^{*}}$ é compacta na topologia $w^{*}$ de $X^{*}$.

\subsection{Espaços estritamente convexos}

Nesta seção, trataremos de maneira elementar do conceito de espaço estritamente convexo. Basearemo-nos em [Meg98] e indicamos esta referência para mais detalhes sobre o assunto.

Dado um espaço normado $X$, é tentador pensar na sua bola unitária fechada $B_{X}$ como uma figura redonda e suave, análoga a um círculo em $\mathbb{R}^{2}$. Porém, não são todos os espaços normados que possuem bolas com essa característica. De fato, bolas em $\ell_{1}^{2}$ e $\ell_{\infty}^{2}$ estão longe de ser assim. 
Nesta seção e na próxima veremos condições que um espaço normado deve satisfazer para que sua bola seja aproximadamente da forma como a imaginamos intuitivamente. O conceito de espaço estritamente convexo, definido abaixo, está relacionado à não-existência de segmentos de reta nãotriviais na esfera do espaço normado, como veremos mais adiante.

Definição 1.4.1. Um espaço normado $X$ é dito estritamente convexo se $\left\|t x_{1}+(1-t) x_{2}\right\|<1$ sempre que $x_{1}$ e $x_{2}$ forem elementos distintos de $S_{X}$ e $t \in(0,1)$.

A seguir, veremos alguns exemplos de espaços normados que são estritamente convexos e outros que não são estritamente convexos.

Exemplo 1.4.2. (a) Os espaços normados $c_{0}, \ell_{\infty}$ e $\ell_{\infty}^{n}$, para $n \geq 2$, não são estritamente convexos.

Para todos os casos, tomando $x_{1}=e_{1}+e_{2}, x_{2}=e_{1}-e_{2}$ e $t=\frac{1}{2}$, obtemos

$$
\left\|\frac{x_{1}}{2}+\frac{x_{2}}{2}\right\|_{\infty}=1
$$

donde segue a conclusão.

(b) Os espaços normados $\ell_{1}$ e $\ell_{1}^{n}$, para $n \geq 2$, não são estritamente convexos.

Basta observar que em ambos os casos

$$
\left\|\frac{e_{1}}{2}+\frac{e_{2}}{2}\right\|_{1}=1
$$

Mostraremos a seguir que todo espaço com produto interno é estritamente convexo. Porém, antes disso, será feita uma caracterização que facilitará este trabalho.

Proposição 1.4.3. Seja $X$ um espaço normado. São equivalentes:

(a) X é estritamente convexo.

(b) $\frac{\|x+y\|}{2}<1$ sempre que $x, y \in S_{X}$ e $x \neq y$.

(c) $\|x+y\|<\|x\|+\|y\|$ para quaisquer $x, y \in X$ linearmente independentes (LI).

Demonstração. (a) $\Rightarrow$ (b) É claro.

(b) $\Rightarrow$ (a) Sejam $t \in(0,1)$ e $x, y \in S_{X}$ tais que $x \neq y$. Se $t=\frac{1}{2}$, vale que $\frac{\|x+y\|}{2}<1$ por hipótese. Suponhamos, sem perda de generalidade, que $0<t<\frac{1}{2}$ (o caso $\frac{1}{2}<t<1$ segue por um argumento análogo). Considere $\eta \doteq 2 t \in(0,1)$. Como $B_{X}$ é convexo, $y \in B_{X}$ e por hipótese $\left(\frac{x}{2}+\frac{y}{2}\right) \in B_{X}^{\mathrm{o}}$, o Lema 1.1.9 implica que $\eta\left(\frac{x}{2}+\frac{y}{2}\right)+(1-\eta) y \in B_{X}^{\mathrm{o}}$. Portanto, $t x+(1-t) y \in B_{X}^{\mathrm{o}}$, ou seja $\|t x+(1-t) y\|<1$, como queríamos mostrar.

(a) $\Rightarrow$ (c) Sejam $x, y \in X$ linearmente independentes. Dessa forma, são ambos não-nulos e $\frac{x}{\|x\|} \neq \frac{y}{\|y\|}$. Assim, por hipótese,

$$
\left\|t \frac{x}{\|x\|}+(1-t) \frac{y}{\|y\|}\right\|<1 \text { para todo } t \in(0,1)
$$


Em particular, para $t=\frac{\|x\|}{\|x\|+\|y\|} \in(0,1)$, vale que

$$
\left\|\frac{x}{\|x\|+\|y\|}+\frac{y}{\|x\|+\|y\|}\right\|<1,
$$

ou seja, $\|x+y\|<\|x\|+\|y\|$.

(c) $\Rightarrow$ (b) Sejam $x, y \in S_{X}$, distintos. Se $x$ e $y$ forem LI, então $\frac{x}{2}$ e $\frac{y}{2}$ também o serão, e por hipótese,

$$
\left\|\frac{x}{2}+\frac{y}{2}\right\|<\frac{1}{2}+\frac{1}{2}=1 .
$$

Vamos assumir então que $x$ e $y$ são linearmente dependentes, isto é, existe $c \in S_{\mathbb{K}}$ tal que $x=c y$. Suponhamos, por absurdo, que

$$
\left\|\frac{x}{2}+\frac{y}{2}\right\|=\left\|\frac{c y}{2}+\frac{y}{2}\right\|=1,
$$

donde obtemos $|c+1|=2$, o que implica

$$
\sqrt{(1+\operatorname{Re}(c))^{2}+\operatorname{Im}(c)^{2}}=2,
$$

ou seja, $\left(\operatorname{Re}(c)^{2}+\operatorname{Im}(c)^{2}\right)+1+2 \operatorname{Re}(c)=2+2 \operatorname{Re}(c)=4$. Assim, $\operatorname{Re}(c)=1$, e dessa forma $c=1$. Absurdo, pois $x_{1}$ e $x_{2}$ eram distintos, encerrando a prova.

Proposição 1.4.4. Todo espaço com produto interno é estritamente convexo.

Demonstração. Seja $X$ um espaço com produto interno. Consideremos $x$ e $y \in S_{X}$ distintos. Pela Lei do Paralelogramo,

$$
\|x+y\|^{2}+\|x-y\|^{2}=2\left(\|x\|^{2}+\|y\|^{2}\right)=4
$$

e assim

$$
\left(\frac{\|x+y\|}{2}\right)^{2}=1-\left(\frac{\|x-y\|}{2}\right)^{2}<1 .
$$

Portanto, $\frac{\|x+y\|}{2}<1$ e a conclusão segue do item (b) da Proposição 1.4.3.

Para finalizar a seção, veremos que a convexidade estrita de um espaço normado $X$ é equivalente à não-existência de segmentos de reta não-triviais em $S_{X}$.

Lembremos que um segmento de reta (fechado) em um espaço normado $X$ é um conjunto do tipo $\left\{t x_{1}+(1-t) x_{2}: t \in[0,1]\right\}$, para $x_{1}, x_{2} \in X$. O segmento é dito não-trivial se $x_{1} \neq x_{2}$. Denotamos

$$
\left[x_{1} ; x_{2}\right] \doteq\left\{t x_{1}+(1-t) x_{2}: t \in[0,1]\right\}
$$

Proposição 1.4.5. Um espaço normado $X$ é estritamente convexo se, e somente se, $S_{X}$ não contêm segmentos de reta não-triviais.

Demonstração. $(\Rightarrow)$ Se $X$ é estritamente convexo, dados $x_{1}, x_{2} \in S_{X}$ distintos, vale que $t x_{1}+(1-$ t) $x_{2} \notin S_{X}$ para todo $t \in(0,1)$. Em particular, $S_{X}$ não contêm $\left[x_{1} ; x_{2}\right]$. 
$(\Leftarrow)$ Dados $x_{1}, x_{2} \in S_{X}$ distintos, por hipótese $S_{X}$ não contêm $\left[x_{1} ; x_{2}\right]$, e então existe $t_{0} \in(0,1)$ tal que $t_{0} x_{1}+\left(1-t_{0}\right) x_{2} \in B_{X}^{o}$. Dado $t \in(0,1)$ arbitrário, vamos mostrar que $\left\|t x_{1}+(1-t) x_{2}\right\|<1$. Suponha que $t<t_{0}$, sem perda de generalidade (um argumento análogo pode ser usado para o caso $\left.t>t_{0}\right)$. Temos que $\frac{t}{t_{0}} \in(0,1)$ e pelo Lema 1.1.9,

$$
\frac{t}{t_{0}}\left(t_{0} x_{1}+\left(1-t_{0}\right) x_{2}\right)+\left(1-\frac{t}{t_{0}}\right) x_{2} \in B_{X}^{\mathrm{o}}
$$

ou seja, $\left\|t x_{1}+(1-t) x_{2}\right\|<1$. Portanto, $X$ é estritamente convexo.

\subsection{Espaços uniformemente convexos}

Nesta seção trataremos do conceito de espaço uniformemente convexo. Novamente, introduziremos o assunto apenas de maneira elementar, com o objetivo de utilizá-lo no capítulo 3 do trabalho. Para mais detalhes, indicamos as referências [Meg98] e [BPT15], nas quais esta seção foi baseada.

Na seção anterior, vimos que em espaços estritamente convexos $X$ os pontos médios de segmentos não-triviais com extremos na esfera unitária $S_{X}$ devem estar em $B_{X}^{o}$, isto é, dentro de $S_{X}$. Porém, podemos ainda nos perguntar o quão dentro da esfera os pontos médios estão, se mantivermos os extremos dos segmentos a uma distância mínima definida. Estes pontos podem se aproximar indefinidamente da esfera unitária, ou sempre se mantêm a uma distância segura dela? Quando ocorre o segundo caso, o espaço é denominado uniformemente convexo, como definimos abaixo.

Definição 1.5.1. Um espaço normado $X$ é dito uniformemente convexo se para todo $\epsilon>0$ existe $\delta(\epsilon)>0$ tal que

$$
x, y \in S_{X}, \frac{\|x+y\|}{2}>1-\delta(\epsilon) \Rightarrow\|x-y\|<\epsilon .
$$

Tendo em vista a Proposição 1.4.3, é claro que todo espaço uniformemente convexo é estritamente convexo. Dessa forma, os espaços $\ell_{1}, \ell_{\infty}, c_{0}, \ell_{1}^{n}$ e $\ell_{\infty}^{n}$, para $n \geq 2$, não são uniformemente convexos, uma vez que não são estritamente convexos, como mostrado na seção anterior.

Observação 1.5.2. Na definição acima, podemos substituir a esfera unitária $S_{X}$ pela bola unitária fechada, $B_{X}$. Em outras palavras, um espaço normado $X$ é uniformemente convexo se, e somente se, para todo $\epsilon>0$ existe $\delta(\epsilon)>0$ tal que

$$
x, y \in B_{X}, \frac{\|x+y\|}{2}>1-\delta(\epsilon) \Rightarrow\|x-y\|<\epsilon
$$

Vamos provar esta afirmação. É claro que se um espaço normado satisfaz a condição acima, então deve ser uniformemente convexo. Resta mostrar o outro lado.

Suponha que $X$ seja uniformemente convexo, e considere $0<\epsilon<1$. Então existe $\delta_{1}>0$ tal que

$$
x, y \in S_{X}, \frac{\|x+y\|}{2}>1-\delta_{1} \Rightarrow\|x-y\|<\frac{\epsilon}{3} .
$$

Mostraremos que tomando $\delta \doteq \min \left\{\frac{\epsilon}{6}, \frac{\delta_{1}}{3}\right\}$, a contrapositiva da condição (1.5.1) é obedecida. 
Suponhamos que $x, y \in B_{X}$ sejam tais que $\|x-y\| \geq \epsilon$. Observemos que se $\|x\| \leq 1-2 \delta$ ou $\|y\| \leq 1-2 \delta$, temos

$$
\frac{\|x+y\|}{2} \leq \frac{\|x\|}{2}+\frac{\|y\|}{2} \leq 1-\delta
$$

como queríamos. Vamos supor então que $1-2 \delta<\|x\|$, $\|y\| \leq 1$. Como $\delta \leq \frac{1}{3}$, $x$ e $y$ são vetores não-nulos e assim podemos tomar

$$
x^{\prime}=\frac{x}{\|x\|}, y^{\prime}=\frac{y}{\|y\|} .
$$

Temos que $\left\|x^{\prime}-x\right\|=1-\|x\|<2 \delta$ e analogamente $\left\|y^{\prime}-y\right\|<2 \delta$, donde vem

$$
\left\|x^{\prime}-y^{\prime}\right\| \geq\|x-y\|-\left\|x^{\prime}-x\right\|-\left\|y^{\prime}-y\right\| \geq \epsilon-4 \delta \geq \epsilon-\frac{4 \epsilon}{6}=\frac{\epsilon}{3} .
$$

Pela escolha de $\delta_{1}$, podemos concluir que $\frac{\left\|x^{\prime}+y^{\prime}\right\|}{2} \leq 1-\delta_{1}$. Portanto,

$$
\begin{aligned}
\frac{\|x+y\|}{2} & =\frac{\left\|x-x^{\prime}+x^{\prime}+y^{\prime}-y^{\prime}+y\right\|}{2} \\
& \leq \frac{\left\|x-x^{\prime}\right\|}{2}+\frac{\left\|x^{\prime}+y^{\prime}\right\|}{2}+\frac{\left\|y^{\prime}-y\right\|}{2} \\
& \leq 2 \delta+1-\delta_{1} \leq 1-\frac{\delta_{1}}{3} \leq 1-\delta,
\end{aligned}
$$

concluindo a demonstração.

A seguir mostramos que espaços com produto interno sempre são uniformemente convexos, resultado que generaliza a Proposição 1.4.4.

Proposição 1.5.3. Todo espaço com produto interno é uniformemente convexo.

Demonstração. Seja $X$ um espaço com produto interno. Dado $\epsilon>0$, se $x, y \in B_{X}$ são tais que $\|x-y\| \geq \epsilon$, pela Lei do Paralelogramo,

$$
\frac{\|x+y\|^{2}}{4}=\frac{\|x\|^{2}}{2}+\frac{\|y\|^{2}}{2}-\frac{\|x-y\|^{2}}{4} \leq 1-\frac{\epsilon^{2}}{4} .
$$

Basta então tomar $\delta(\epsilon) \doteq 1-\sqrt{1-\frac{\epsilon^{2}}{4}}$ para dado $\epsilon>0$ na Definição 1.5.1 (notemos que se $\|x-y\| \geq \epsilon$ com $x, y \in B_{X}$, obrigatoriamente $\left.\epsilon \leq 2\right)$.

Apresentamos agora uma caracterização de espaços uniformemente convexos usada com frequência.

Proposição 1.5.4. Um espaço normado $X$ é uniformemente convexo se, e somente se, para quaisquer sequências $\left(x_{n}\right)_{n \in \mathbb{N}}$ e $\left(y_{n}\right)_{n \in \mathbb{N}}$ em $S_{X}$ satisfazendo a $\left\|\frac{1}{2}\left(x_{n}+y_{n}\right)\right\| \rightarrow 1$, vale que $\left\|x_{n}-y_{n}\right\| \rightarrow$ 0 . 
Demonstração. $(\Rightarrow)$ Por hipótese, dado $\epsilon>0$ existe $\delta(\epsilon)>0$ tal que

$$
x, y \in B_{X}, \frac{\|x+y\|}{2}>1-\delta(\epsilon) \Rightarrow\|x-y\|<\epsilon
$$

Sejam $\left(x_{n}\right)_{n \in \mathbb{N}}$ e $\left(y_{n}\right)_{n \in \mathbb{N}}$ sequências em $S_{X}$ tais que $\left\|\frac{1}{2}\left(x_{n}+y_{n}\right)\right\| \rightarrow 1$. Dessa forma, para dado $\epsilon>0$, existe $n_{0} \in \mathbb{N}$ tal que

$$
n \geq n_{0} \Rightarrow \frac{\left\|x_{n}+y_{n}\right\|}{2}>1-\delta(\epsilon) \Rightarrow\left\|x_{n}-y_{n}\right\|<\epsilon,
$$

o que encerra a demonstração deste lado.

$(\Leftarrow)$ Suponha que $X$ não seja uniformemente convexo. Então existe $\epsilon_{0}>0$ tal que, para todo $n \in \mathbb{N}$, existem $x_{n}$ e $y_{n}$ em $S_{X}$ tais que

$$
\frac{\left\|x_{n}+y_{n}\right\|}{2}>1-\frac{1}{n} \mathrm{e}\left\|x_{n}-y_{n}\right\| \geq \epsilon_{0}
$$

Absurdo, pois nesse caso teríamos que $\left\|\frac{1}{2}\left(x_{n}+y_{n}\right)\right\| \rightarrow 1$ mas $\left\|x_{n}-y_{n}\right\| \nrightarrow 0$.

Dois conceitos relacionados que também serão usados no capítulo 3 do trabalho são os de ponto e espaço localmente uniformemente convexo, definidos abaixo.

Definição 1.5.5. Seja $X$ um espaço normado. Um ponto $x \in S_{X}$ é dito localmente uniformemente convexo (ponto LUR) se para cada $\epsilon>0$ existe $\delta(\epsilon)>0$ tal que

$$
y \in S_{X}, \frac{\|x+y\|}{2}>1-\delta(\epsilon) \Rightarrow\|x-y\|<\epsilon
$$

Definição 1.5.6. Um espaço normado $X$ é dito localmente uniformemente convexo (espaço LUR) se todo ponto de $S_{X}$ é ponto LUR.

É claro que se um espaço normado $X$ é uniformemente convexo, então todo ponto da sua esfera unitária é LUR, ou seja, $X$ é LUR. A recíproca não vale em geral, já que no caso dos espaços LUR para cada $\epsilon>0$ existe uma constante $\delta(\epsilon)$ para cada ponto da esfera unitária considerado. Observemos também que todo espaço LUR é estritamente convexo.

\subsection{Somabilidade em espaços normados}

Séries em espaços normados tratam-se de limites de somas finitas. Sabemos, por exemplo, que elas dependem em geral da ordem escolhida para somar. Uma pergunta relacionada a este tema é se há uma forma de definir somas em conjuntos de índices arbitrários, enumeráveis ou não. Seria interessante que essa soma dependesse apenas da família a ser somada, e não mais da ordem. O conceito de somabilidade é uma forma de fazer isso. Este conceito permite ainda obter demonstrações de propriedades importantes de séries.

Procuraremos nesta seção introduzir a somabilidade e demonstrar algumas de suas propriedades. Em seguida, aplicaremos tais resultados para obter propriedades do espaço de Banach $c_{0}$, usado com frequência ao longo do trabalho, principalmente no capítulo 3. 
Sejam $X$ um espaço normado, $I$ um conjunto e $\left(x_{i}\right)_{i \in I}$ uma família em $X$. Definamos

$$
\Lambda \doteq\{F \subset I: F \text { é finito }\}
$$

e

$$
\begin{aligned}
S: \Lambda & \rightarrow X \\
F & \mapsto \sum_{i \in F} x_{i} .
\end{aligned}
$$

Observemos aqui que por definição $\sum_{i \in \emptyset} x_{i} \doteq 0$. Se considerarmos $\preceq$ como sendo a relação de inclusão em $\Lambda$, isto é,

$$
\left(F_{1} \preceq F_{2} \Leftrightarrow F_{1} \subset F_{2}\right) \text { para todos } F_{1}, F_{2} \in \Lambda,
$$

temos que $(\Lambda, \preceq)$ é um conjunto dirigido e $S$ é uma rede em $X$.

Usaremos a notação dada acima no decorrer desta seção. Sendo assim, a menos que se diga o contrário, $X$ é um espaço normado sobre $\mathbb{K}, I$ é um conjunto e $\left(x_{i}\right)_{i \in I}$ é uma família em $X$. O conjunto $\Lambda$ será dirigido pela relação de inclusão e $S$ é a rede definida anteriormente. Denotaremos esta rede por $\left(S_{F}\right)_{F \in \Lambda}$.

Definição 1.6.1. A família $\left(x_{i}\right)_{i \in I}$ é dita somável se a rede $\left(S_{F}\right)_{F \in \Lambda}$ converge.

Observação 1.6.2. Note que, se existir, o limite da rede $\left(S_{F}\right)_{F \in \Lambda}$ é único, pois $X$ é espaço de Hausdorff.

A Proposição abaixo apenas traduz a convergência da rede $\left(S_{F}\right)_{F \in \Lambda}$ :

Proposição 1.6.3. São equivalentes:

(i) A família $\left(x_{i}\right)_{i \in I}$ é somável e $S_{F} \rightarrow x$.

(ii) Para todo $\epsilon>0$, existe $F_{0} \subset I$ finito tal que se $F \supset F_{0}$ for finito, então $\left\|\sum_{i \in F} x_{i}-x\right\|<\epsilon$.

Note que se $I$ for finito qualquer família $\left(x_{i}\right)_{i \in I}$ em $X$ é somável e vale que $\lim S_{F}=\sum_{i \in I} x_{i}$. Então, sem perigo de confusão no caso de índices finitos, podemos a partir de agora denotar $\lim S_{F} \doteq$ $\sum_{i \in I} x_{i}$, para qualquer conjunto de índices $I$ e família $\left(x_{i}\right)_{i \in I}$ em $X$, no caso da família ser somável.

Proposição 1.6.4. Sejam $I_{1}, I_{2} \subset I$ tais que $I_{1} \cap I_{2}=\emptyset$ e $I_{1} \cup I_{2}=I$. Se $\left(x_{i}\right)_{i \in I_{1}}$ e $\left(x_{i}\right)_{i \in I_{2}}$ são somáveis, então $\left(x_{i}\right)_{i \in I}$ é somável e $\sum_{i \in I_{1}} x_{i}+\sum_{i \in I_{2}} x_{i}=\sum_{i \in I} x_{i}$.

Demonstração. Considere $\epsilon>0$. Como $\left(x_{i}\right)_{i \in I_{1}}$ e $\left(x_{i}\right)_{i \in I_{2}}$ são somáveis, existem $F_{0}^{1}$ e $F_{0}^{2}$ subconjuntos finitos de $I_{1}$ e $I_{2}$, respectivamente, tais que

$$
\begin{aligned}
& I_{1} \supset F_{1} \supset F_{0}^{1}, F_{1} \text { finito } \Rightarrow\left\|\sum_{i \in F_{1}} x_{i}-\sum_{i \in I_{1}} x_{i}\right\|<\frac{\epsilon}{2} ; \\
& I_{2} \supset F_{2} \supset F_{0}^{2}, F_{2} \text { finito } \Rightarrow\left\|\sum_{i \in F_{2}} x_{i}-\sum_{i \in I_{2}} x_{i}\right\|<\frac{\epsilon}{2} .
\end{aligned}
$$


Definamos $F_{0} \doteq F_{0}^{1} \cup F_{0}^{2}$ e seja $F \supset F_{0}$ subconjunto finito de $I$. Notemos que $F=\left(F \cap I_{1}\right) \cup\left(F \cap I_{2}\right)$ e $\left(F \cap I_{1}\right) \cap\left(F \cap I_{2}\right)=\emptyset$, donde vem

$$
\sum_{i \in F} x_{i}=\sum_{i \in F \cap I_{1}} x_{i}+\sum_{i \in F \cap I_{2}} x_{i}
$$

Como $F \cap I_{1} \supset F_{0}^{1}$ e $F \cap I_{2} \supset F_{0}^{2}$, por (1.6.1),

$$
\begin{aligned}
& \left\|\sum_{i \in F \cap I_{1}} x_{i}-\sum_{i \in I_{1}} x_{i}\right\|<\frac{\epsilon}{2} ; \\
& \left\|\sum_{i \in F \cap I_{2}} x_{i}-\sum_{i \in I_{2}} x_{i}\right\|<\frac{\epsilon}{2} .
\end{aligned}
$$

Pelas desigualdes acima e (1.6.2), obtemos

$$
\left\|\sum_{i \in F} x_{i}-\left(\sum_{i \in I_{1}} x_{i}+\sum_{i \in I_{2}} x_{i}\right)\right\|<\epsilon,
$$

donde segue a conclusão desejada.

Quando $X$ é um espaço de Banach, vale um pouco mais:

Proposição 1.6.5. Sejam $X$ um espaço de Banach e I um conjunto. Suponha que a familia $\left(x_{i}\right)_{i \in I}$ em $X$ seja somável. Se $I_{1}, I_{2} \subset I$ são tais que $I_{1} \cap I_{2}=\emptyset$ e $I_{1} \cup I_{2}=I$, então $\left(x_{i}\right)_{i \in I_{1}}$ e $\left(x_{i}\right)_{i \in I_{2}}$ são somáveis e $\sum_{i \in I_{1}} x_{i}+\sum_{i \in I_{2}} x_{i}=\sum_{i \in I} x_{i}$.

Demonstração. Para $j=1,2$, sejam $\Lambda^{(j)}=\left\{F \subset I_{j}: F\right.$ é finito $\}$ e $S^{(j)}: \Lambda^{(j)} \ni F \mapsto \sum_{i \in F} x_{i} \in X$. Vamos verificar que $\left(x_{i}\right)_{i \in I_{1}}$ é somável, mostrando que a rede $S^{(1)}$ é de Cauchy. Para isso, considere $\epsilon>0$. Como $\left(x_{i}\right)_{i \in I}$ é somável, existe $F_{0} \subset I$ finito tal que se $F_{1}, F_{2} \supset F_{0}$ são finitos, então

$$
\left\|\sum_{i \in F_{1}} x_{1}-\sum_{i \in F_{2}} x_{i}\right\|<\epsilon
$$

Seja $F_{0}^{\prime} \doteq F_{0} \cap I_{1}$. Dados $F_{1}^{\prime}, F_{2}^{\prime} \supset F_{0}^{\prime}$ subconjuntos finitos de $I_{1}$, vale que

$$
\left\|\sum_{i \in F_{1}^{\prime}} x_{1}-\sum_{i \in F_{2}^{\prime}} x_{i}\right\|=\left\|\sum_{i \in F_{1}^{\prime} \cup\left(F_{0} \backslash I_{1}\right)} x_{i}-\sum_{i \in F_{2}^{\prime} \cup\left(F_{0} \backslash I_{1}\right)} x_{i}\right\| .
$$

Observando que $F_{1}^{\prime} \cup\left(F_{0} \backslash I_{1}\right)$ e $F_{2}^{\prime} \cup\left(F_{0} \backslash I_{1}\right)$ são subconjuntos finitos de $I$ que contêm $F_{0}$, por (1.6.3) teremos

$$
\left\|\sum_{i \in F_{1}^{\prime}} x_{1}-\sum_{i \in F_{2}^{\prime}} x_{i}\right\|<\epsilon .
$$

Portanto, a rede $S^{(1)}$ é de Cauchy, e $\left(x_{i}\right)_{i \in I_{1}}$ é somável, uma vez que $X$ é espaço de Banach. Um argumento análogo mostra que $\left(x_{i}\right)_{i \in I_{2}}$ também é somável. Pela Proposição 1.6.4, segue que $\sum_{i \in I_{1}} x_{i}+\sum_{i \in I_{2}} x_{i}=\sum_{i \in I} x_{i}$. 
A Proposição abaixo dá uma condição suficiente para que uma família indexada nos naturais seja somável, usando a convergência absoluta de séries.

Proposição 1.6.6. Se $X$ é um espaço de Banach e $\left(x_{i}\right)_{i \in \mathbb{N}}$ é uma família em $X$ tal que $\sum_{i=1}^{\infty}\left\|x_{i}\right\|<$ $\infty$, então $\left(x_{i}\right)_{i \in \mathbb{N}}$ é somável.

Demonstração. Vamos mostrar que a rede $\left(S_{F}\right)_{F \in \Lambda}$ é de Cauchy. Para isso, considere $\epsilon>0$. Então existe $i_{0} \in \mathbb{N}$ tal que para quaisquer $M, N \in \mathbb{N}$,

$$
M>N \geq i_{0} \Rightarrow \sum_{i=N+1}^{M}\left\|x_{i}\right\|<\epsilon .
$$

Seja $F_{0}=\left\{1, \ldots, i_{0}\right\}$. Se $F_{1}, F_{2} \supset F_{0}$ são finitos, temos

$$
\left\|\sum_{i \in F_{1}} x_{i}-\sum_{i \in F_{2}} x_{i}\right\|=\left\|\sum_{i \in F_{1}} x_{i}+\sum_{i \in F_{2}}-x_{i}\right\|=\left\|\sum_{i \in F_{1} \cup F_{2}} y_{i}\right\|,
$$

em que

$$
y_{i}= \begin{cases}x_{i}, & \text { se } i \in F_{1} \backslash F_{2} \\ 0, & \text { se } i \in F_{1} \cap F_{2} \\ -x_{i}, & \text { se } i \in F_{2} \backslash F_{1} .\end{cases}
$$

Logo,

$$
\begin{aligned}
\left\|\sum_{i \in F_{1}} x_{i}-\sum_{i \in F_{2}} x_{i}\right\| & \leq \sum_{i \in F_{1} \cup F_{2}}\left\|y_{i}\right\| \\
& =\sum_{i \in\left(F_{1} \cup F_{2}\right) \backslash F_{0}}\left\|y_{i}\right\| \\
& \leq \sum_{i \in\left(F_{1} \cup F_{2}\right) \backslash F_{0}}\left\|x_{i}\right\| .
\end{aligned}
$$

Seja $M_{0}>\operatorname{máx}\left\{n: n \in F_{1} \cup F_{2}\right\} \geq i_{0}$. Então

$$
\begin{aligned}
\left\|\sum_{i \in F_{1}} x_{i}-\sum_{i \in F_{2}} x_{i}\right\| & \leq \sum_{i \in\left(F_{1} \cup F_{2}\right) \backslash F_{0}}\left\|x_{i}\right\| \\
& \leq \sum_{\substack{M_{0} \\
M_{0}+1}}\left\|x_{i}\right\| \\
& <\epsilon .4 .
\end{aligned}
$$

Portanto, $\left(S_{F}\right)_{F \in \Lambda}$ é rede de Cauchy e, como $X$ é espaço de Banach, converge. Assim, $\left(x_{i}\right)_{i \in \mathbb{N}}$ é somável.

Se $\left(x_{i}\right)_{i \in \mathbb{N}}$ é uma família em $X$, chamamos de série formal a sequência das somas parciais $\left(\sum_{i=1}^{n} x_{i}\right)_{n \in \mathbb{N}}$. A série formal de $\left(x_{i}\right)_{i \in \mathbb{N}}$ é denotada $\sum_{n} x_{n}$, ou mesmo $\sum_{i=1}^{\infty} x_{i}$, quando não há perigo de confusão. 
Definição 1.6.7. Sejam $X$ um espaço normado e $\left(x_{i}\right)_{i \in \mathbb{N}}$ uma família em $X$. A série formal $\sum_{n} x_{n}$ é dita incondicionalmente convergente se para toda permutação $\pi$ de $\mathbb{N}$ vale que $\sum_{n} x_{\pi(n)}$ converge.

É bem conhecido que se uma série formal $\sum_{n} x_{n}$ em $\mathbb{R}$ ou $\mathbb{C}$ é incondicionalmente convergente, então $\sum_{i=1}^{\infty} x_{i}=\sum_{i=1}^{\infty} x_{\pi(i)}$ para toda $\pi$ permutação de $\mathbb{N}$. Na verdade, isso vale para espaços normados em geral, como veremos abaixo.

Proposição 1.6.8. Se $X$ é um espaço normado e a série formal $\sum_{n} x_{n}$ é incondicionalmente convergente, então $\sum_{i=1}^{\infty} x_{i}=\sum_{i=1}^{\infty} x_{\pi(i)}$ para toda permutação $\pi$ de $\mathbb{N}$.

Demonstração. Como $\sum_{n} x_{n}$ é incondicionalmente convergente, dados $f \in X^{*}$ e $\pi$ permutação de $\mathbb{N}, f\left(\sum_{i=1}^{\infty} x_{\pi(i)}\right)=\sum_{i=1}^{\infty} f\left(x_{\pi(i)}\right)$. Em particular, $\sum_{i=1}^{\infty} f\left(x_{\pi(i)}\right)$ converge para toda permutação $\pi$ de $\mathbb{N}$. Portanto, a série formal $\sum_{n} f\left(x_{n}\right)$ em $\mathbb{K}$ é incondicionalmente convergente e, como já observado, isso implica que $\sum_{i=1}^{\infty} f\left(x_{i}\right)=\sum_{i=1}^{\infty} f\left(x_{\pi(i)}\right)$ para toda permutação $\pi$. Mostramos assim que dada uma permutação $\pi: \mathbb{N} \rightarrow \mathbb{N}$, vale que $f\left(\sum_{=1}^{\infty} x_{i}\right)=f\left(\sum_{i=1}^{\infty} x_{\pi(i)}\right)$ para todo $f \in X^{*}$, e pelo Corolário 1.1.17, $\sum_{=1}^{\infty} x_{i}=\sum_{i=1}^{\infty} x_{\pi(i)}$.

Dada uma família $\left(x_{i}\right)_{i \in \mathbb{N}}$ no espaço normado $X$, a priori poderia não existir relação entre a sua somabilidade e a convergência da série formal $\sum_{n} x_{n}$. Mesmo que a família seja somável e a série convergente, será verdade que $\sum_{i \in \mathbb{N}} x_{i}=\sum_{i=1}^{\infty} x_{i}$ ? Veremos na proposição seguinte que a somabilidade da família é equivalente à convergência incondicional da série formal $\sum_{n} x_{n}$, e nesse caso teremos a igualdade dos dois valores de soma. Portanto, o conceito de somabilidade é mais forte do que a simples convergência, no caso do conjunto de índices $\mathbb{N}$.

Proposição 1.6.9. Seja $X$ um espaço normado. Uma família $\left(x_{i}\right)_{i \in \mathbb{N}}$ em $X$ é somável se, e somente se, a série formal $\sum_{n} x_{n}$ é incondicionalmente convergente. Nesse caso, vale que $\sum_{i \in \mathbb{N}} x_{i}=\sum_{i=1}^{\infty} x_{i}$.

Demonstração. $(\Rightarrow)$ Suponhamos que $\left(x_{i}\right)_{i \in \mathbb{N}}$ seja somável. Considere $\pi: \mathbb{N} \rightarrow \mathbb{N}$ uma permutação. Para cada $n \in \mathbb{N}$, definamos $M_{n} \doteq\{\pi(1), \ldots, \pi(n)\}$ e a aplicação

$$
\begin{aligned}
M: \mathbb{N} & \rightarrow \Lambda \\
n & \mapsto M_{n} .
\end{aligned}
$$

Considerando $\mathbb{N}$ munido da sua ordem usual, a função $M$ é crescente e cofinal. Teremos então que a composição $S \circ M$ é uma subrede de $S$. Denotamos tal subrede por $\left(S_{F_{n}}\right)_{n \in \mathbb{N}}$. Assim, vale que $\lim S_{F_{n}}=\sum_{i \in \mathbb{N}} x_{i}$, ou seja, dado $\epsilon>0$, existe $n_{0} \in \mathbb{N}$ tal que

$$
n \geq n_{0} \Rightarrow\left\|\sum_{i \in M_{n}} x_{i}-\sum_{i \in \mathbb{N}} x_{i}\right\|=\left\|\sum_{i=1}^{n} x_{\pi(i)}-\sum_{i \in \mathbb{N}} x_{i}\right\|<\epsilon .
$$

Portanto, $\sum_{i \in \mathbb{N}} x_{i}=\sum_{i=1}^{\infty} x_{\pi(i)}$ para toda $\pi: \mathbb{N} \rightarrow \mathbb{N}$ permutação. Em particular, $\sum_{n} x_{n}$ é incondicionalmente convergente.

$(\Leftarrow)$ Assumamos que $\sum_{n} x_{n}$ é incondicionalmente convergente. Suponha, por absurdo, que a família $\left(x_{i}\right)_{i \in \mathbb{N}}$ não seja somável a $\sum_{i=1}^{\infty} x_{i}$. Então existe $\epsilon>0$ tal que para cada $F \subset \mathbb{N}$ finito, existe $c(F) \subset \mathbb{N}$ finito satisfazendo a

$$
F \subset c(F) \text { e }\left\|\sum_{i \in c(F)} x_{i}-\sum_{i=1}^{\infty} x_{i}\right\| \geq \epsilon
$$


Vamos definir recursivamente uma sequência $\left(c_{i}\right)_{i \in \mathbb{N}}$ de subconjuntos de $\mathbb{N}$ da seguinte forma:

$$
\begin{gathered}
c_{1} \doteq c(\{1\}) ; \\
c_{n} \doteq c\left(c_{n-1} \cup\{n\}\right) \text { se } n>1 .
\end{gathered}
$$

Por construção, para todo $n \in \mathbb{N}$ vale que:

(I) $c_{n}$ é conjunto finito;

(II) $n \in c_{n}$;

(III) $c_{n-1} \subset c_{n}$, se $n>1$;

(IV) $\left\|\sum_{i \in c_{n}} x_{i}-\sum_{i=1}^{\infty} x_{i}\right\| \geq \epsilon$.

Descreveremos agora uma listagem dos naturais. Primeiro, listemos os elementos de $c_{1}$ em ordem crescente. Em seguida, os elementos de $c_{2}$ ainda não listados, também em ordem crescente. Depois, os de $c_{3}$, e assim sucessivamente. Seja $\sigma: \mathbb{N} \rightarrow \mathbb{N}$ a permutação que lista $\mathbb{N}$ dessa maneira. Notemos que, devido a (III), se $E_{n}$ denotar o número de elementos de $c_{n}$, então $\sigma\left(\left\{1, \ldots, E_{n}\right\}\right)=c_{n}$, para cada $n \in \mathbb{N}$.

Por hipótese e pela Proposição 1.6.8, vale que $\sum_{i=1}^{\infty} x_{i}=\sum_{i=1}^{\infty} x_{\sigma(i)}$. Logo, existe $N \in \mathbb{N}$ tal que

$$
n \geq N \Rightarrow\left\|\sum_{i=1}^{n} x_{\sigma(i)}-\sum_{i=1}^{\infty} x_{i}\right\|<\epsilon .
$$

Seja $E_{N}$ o número de elementos de $c_{N}$. Por (II) e (III), $\{1, \ldots, N\} \subset c_{N}$, e então $E_{N} \geq N$. Além disso, como já observado, $\sum_{i \in c_{N}} x_{i}=\sum_{i=1}^{E_{N}} x_{\sigma(i)}$. Tendo em vista (1.6.5) e (IV), chegamos a uma contradição. Logo, $\left(x_{i}\right)_{i \in \mathbb{N}}$ é somável e $\sum_{i \in \mathbb{N}} x_{i}=\sum_{i=1}^{\infty} x_{i}$.

É claro que o conteúdo das Proposições 1.6.6, 1.6.8 e 1.6.9 continua verdadeiro, com os ajustes adequados, se o conjunto $\mathbb{N}$ for trocado por um conjunto infinito enumerável qualquer:

Proposição 1.6.10. Se $X$ é um espaço de Banach, A é um conjunto infinito enumerável e $\left(x_{i}\right)_{i \in A}$ é uma família em $X$ tal que $\sum_{i=1}^{\infty}\left\|x_{\pi(i)}\right\|<\infty$ para alguma $\pi: \mathbb{N} \rightarrow A$ bijeção, então $\left(x_{i}\right)_{i \in A}$ é somável.

Proposição 1.6.11. Sejam $X$ um espaço normado, A um conjunto infinito enumerável $e\left(x_{i}\right)_{i \in A}$ uma família em $X$. São equivalentes:

(i) $\left(x_{i}\right)_{i \in A}$ é somável.

(ii) $\sum_{i=1}^{\infty} x_{\pi(i)}$ converge para toda $\pi: \mathbb{N} \rightarrow A$ bijeção.

Nesse caso, $\sum_{i \in A} x_{i}=\sum_{i=1}^{\infty} x_{\pi(i)}$ para qualquer $\pi: \mathbb{N} \rightarrow A$ bijeção.

Notemos agora que das Proposições 1.6.6 e 1.6.9 segue o seguinte Corolário.

Corolário 1.6.12. Se $X$ um espaço de Banach e $\left(x_{i}\right)_{i \in \mathbb{N}}$ é uma familia em $X$ tal que $\sum_{i=1}^{\infty}\left\|x_{i}\right\|<$ $\infty$, então $\sum_{n} x_{n}$ é incondicionalmente convergente. 
A recíproca do Corolário anterior valerá se, e somente se, o espaço de Banach $X$ tiver dimensão finita. Este resultado é consequência do famoso Teorema de Dvoretzky-Rogers (vide por exemplo [BPT15]).

No que segue, aplicaremos os resultados obtidos acima para o espaço de Banach $c_{0}$, e obteremos algumas propriedades que serão usadas no trabalho. De agora em diante, para tornar a notação mais limpa, denotaremos por $\|$.$\| a norma de elementos de c_{0}$, em vez de escrever sempre $\|.\|_{\infty}$.

Para começar, lembremos a definição de base de Schauder em um espaço de Banach:

Definição 1.6.13. Uma sequência $\left(x_{n}\right)_{n \in \mathbb{N}}$ em um espaço de Banach $X$ é dita uma base de Schauder para $X$ se, para todo $x \in X$, existir uma única sequência $\left(\alpha_{n}\right)_{n \in \mathbb{N}}$ em $\mathbb{K}$ tal que $x=\sum_{n=1}^{\infty} \alpha_{n} x_{n}$.

Lembremos ainda que dado $n \in \mathbb{N}, e_{n}$ denota a sequência que tem como i-ésima coordenada $\delta_{n i}$, para todo $i \in \mathbb{N}$. É bem conhecido que a sequência $\left(e_{n}\right)_{n \in \mathbb{N}}$ é uma base de Schauder para $c_{0}$. De fato, dado $x \in c_{0},\left(x_{n}\right)_{n \in \mathbb{N}}$ é a única sequência em $\mathbb{K}$ tal que $x=\sum_{n=1}^{\infty} x_{n} e_{n}$. Para essa base de Schauder vale ainda uma propriedade adicional:

Proposição 1.6.14. Dado $x \in c_{0}$, a série formal $\sum_{n} x_{n} e_{n}$ é incondicionalmente convergente.

Demonstração. Seja $\pi: \mathbb{N} \rightarrow \mathbb{N}$ uma permutação. Vamos mostrar que $\sum_{i=1}^{\infty} x_{\pi(n)} e_{\pi(n)}$ converge. Considere $\epsilon>0$. Como $x \in c_{0}$, existe $n_{0} \in \mathbb{N}$ tal que $\left|x_{n}\right|<\epsilon$ sempre que $n \geq n_{0}$. Tome $m \in \mathbb{N}$ tal que $\left\{1, \ldots, n_{0}\right\} \subset \pi(\{1, \ldots, m\})$. É claro que, nesse caso, $n>m$ implica $\pi(n)>n_{0}$. Assim, se $m_{0}, m_{1} \in \mathbb{N}$ forem tais que $m_{0}>m_{1}>m$, então

$$
\begin{aligned}
\left\|\sum_{n=1}^{m_{0}} x_{\pi(n)} e_{\pi(n)}-\sum_{n=1}^{m_{1}} x_{\pi(n)} e_{\pi(n)}\right\| & =\left\|\sum_{n=m_{1}+1}^{m_{0}} x_{\pi(n)} e_{\pi(n)}\right\| \\
& =\sup _{m_{1}+1 \leq n \leq m_{0}}\left|x_{\pi(n)}\right| \\
& <\epsilon .
\end{aligned}
$$

Portanto, a sequência das somas parciais $\left(\sum_{n} x_{\pi(n)} e_{\pi(n)}\right)_{n \in \mathbb{N}}$ é de Cauchy. Como $c_{0}$ é espaço de Banach, segue que $\sum_{i=1}^{\infty} x_{\pi(n)} e_{\pi(n)}$ converge para toda permutação $\pi$ e, portanto, a série formal $\sum_{n} x_{n} e_{n}$ é incondicionalmente convergente.

A Proposição acima combinada com a Proposição 1.6.9 nos permite concluir que para todo $x \in c_{0}$ a família $\left(x_{n} e_{n}\right)_{n \in \mathbb{N}}$ é somável. Isso torna possível definir, para um subconjunto qualquer $A \subset \mathbb{N}$, a aplicação $P_{A}: c_{0} \rightarrow c_{0}$ dada por $P_{A}(x)=\sum_{n \in A} x_{n} e_{n}$ para cada $x \in c_{0}$, uma vez que a Proposição 1.6.5 garante que $\left(x_{n} e_{n}\right)_{n \in A}$ é somável.

Vamos verificar a seguir que para todos $x \in c_{0}$ e $n \in \mathbb{N}$,

$$
\left(P_{A}(x)\right)_{n}= \begin{cases}x_{n}, & \text { se } n \in A \\ 0, & \text { se } n \notin A .\end{cases}
$$

Para $x \in c_{0}$, seja $y \in c_{0}$ tal que $y_{n}=x_{n}$ se $n \in A$ e $y_{n}=0$ se $n \notin A$. Dado $\epsilon>0$, existe $n_{0} \in \mathbb{N}$ tal que $\left|x_{n}\right|<\epsilon$ se $n \geq n_{0}$. Seja $F_{0} \doteq\left\{1, \ldots, n_{0}\right\} \cap A$. Então se $F \supset F_{0}$ for um subconjunto finito de $A$, teremos

$$
\left\|\sum_{n \in F} x_{n} e_{n}-y\right\|=\sup _{n \in A \backslash F}\left|y_{n}\right| \leq \epsilon,
$$


o que garante que $\sum_{n \in A} x_{n} e_{n}=y$, como queríamos.

Por fim, notemos que para todo $A \subset \mathbb{N}$ vale que $P_{A}$ é linear, pelas propriedades de soma e produto por escalar de limites de redes em espaços normados (vide Proposição 1.2.10), e também $P_{A} \in \mathcal{L}\left(c_{0}, c_{0}\right)$. Além disso, se $A \neq \emptyset$ teremos $\left\|P_{A}\right\|=1$ e se $A=\emptyset, P_{A}=0$. 


\section{Capítulo 2}

\section{Funcionais que atingem a norma e seus teoremas clássicos}

Dado um espaço normado $X$, dizemos que o funcional $f \in X^{*}$ atinge sua norma se existe $x_{0} \in B_{X}$ tal que $\left|f\left(x_{0}\right)\right|=\|f\|$. Definimos ainda o conjunto

$$
\mathcal{N} \mathcal{A}_{X} \doteq\left\{f \in X^{*}: f \text { atinge sua norma }\right\}
$$

Suponhamos agora que $X$ seja um espaço de Banach. Um fato simples de notar é que se $X$ é reflexivo, então $\mathcal{N} \mathcal{A}_{X}=X^{*}$. De fato, o funcional nulo trivialmente atinge sua norma, e dado $f \in X^{*} \backslash\{0\}$, pelo Corolário 1.1.16, existe $\pi \in X^{* *}$ tal que $\|\pi\|=1$ e $\pi(f)=\|f\|$. Como $X$ é reflexivo, existe $x \in X$ tal que $\pi=C_{X}(x)$. Então $1=\left\|C_{X}(x)\right\|=\|x\|$ e $\|f\|=\left|C_{X}(x)(f)\right|=|f(x)|$, ou seja, $f$ atinge sua norma em $x \in B_{X}$. A recíproca deste resultado é o famoso Teorema de James, demonstrado por R. James primeiro para espaços de Banach separáveis [Jam57], em 1957, e depois para o caso geral, em 1964 [Jam64].

Teorema de James. Um espaço de Banach $X$ é reflexivo se, e somente se, todo funcional de $X^{*}$ atinge sua norma.

Não apresentaremos a demonstração do Teorema de James, pois esta é muito técnica e possui detalhes que fogem do escopo deste trabalho.

Inspirados pelo trabalho de James, R. Phelps e E. Bishop também começam a estudar funcionais que atingem a norma. Phelps percebeu que todo espaço de Banach clássico (isto é, os espaços da forma $C(K)$ ou $L_{p}(\mu)$, com $K$ um compacto Hausdorff e $\mu$ uma medida) tinha a seguinte propriedade: o conjunto dos funcionais lineares e contínuos que atingem a norma é denso no dual. Em vista dos resultados de James, parecia a ele que espaços com tal propriedade eram quase reflexivos, razão pela qual ele os chamou de subreflexivos [Phe57]. Porém, um pouco depois, junto com Bishop, viria a descobrir que todo espaço de Banach é subreflexivo, resultado que ficou conhecido como Teorema de Bishop-Phelps [BP61].

Uma versão quantitativa do Teorema de Bishop-Phelps, que ficou conhecida como Teorema de Bishop-Phelps-Bollobás, foi demonstrada por B. Bollobás em 1970 [Bol70]. Mais precisamente, Bollobás mostrou que para qualquer $0<\epsilon<1$, se $x \in S_{X}$ e $f \in S_{X^{*}}$ obedecem a $|1-f(x)|<\epsilon^{2} / 4$, é possível encontrar elementos $y \in S_{X}$ e $g \in S_{X^{*}}$ distando de menos de $\epsilon$ de $x$ e $f$, respectivamente, 
e tais que $g$ atinge sua norma em $y$.

Na primeira seção deste capítulo estudaremos conceitos e resultados elementares a respeito de funcionais que atingem a norma. Na segunda, apresentamos uma demonstração do Teorema de Bishop-Phelps e, na última, do Teorema de Bishop-Phelps-Bollobás. Muitas das demonstrações e resultados aqui apresentados foram baseados em [Meg98].

\subsection{Conceitos iniciais}

Nesta seção estudaremos os conceitos básicos que serão utilizados no decorrer do capítulo. Para começar, definiremos o conceito de funcional suporte, noção que de certa forma generaliza a ideia de funcional que atinge a norma.

Definição 2.1.1. Sejam $X$ um espaço normado e $A \subset X$. Dizemos que $f \in X^{*} \backslash\{0\}$ é um funcional suporte para $A$ (ou que $f$ suporta $A$ ) se existe $x_{0} \in A$ tal que $\operatorname{Re} f\left(x_{0}\right)=\sup \{\operatorname{Re} f(x): x \in A\}$.

Para $X$ e $A$ nas condições da definição anterior, se $x_{0} \in A$ for um ponto tal que existe $f \in X^{*} \backslash\{0\}$ satisfazendo a $\operatorname{Re} f\left(x_{0}\right)=\sup \{\operatorname{Re} f(x): x \in A\}$, diz-se também que $x_{0}$ é ponto suporte para $A$, ou que $f$ suporta $A$ em $x_{0}$.

Quando tratamos de espaços normados sobre $\mathbb{R}$, tomar a parte real de $f(x)$ é redundante, mas em alguns casos manteremos a notação Re $f(x)$ por questões de pragmatismo e clareza, uma vez que muitos dos resultados e demonstrações seguintes valerão tanto se $X$ é espaço vetorial sobre $\mathbb{R}$ quanto sobre $\mathbb{C}$. Quando não for o caso, será explicitado.

Observação 2.1.2. Notemos que segue imediatamente da definição que se um espaço normado $X$ tem um ponto suporte para algum subconjunto, então $X \neq\{0\}$.

Antes da proposição seguinte, observemos que se $x_{0} \in B_{X}$ e $f \in X^{*}$, podemos encontrar um elemento $y_{0} \in B_{X}$ tal que $\left|f\left(x_{0}\right)\right|=\operatorname{Re} f\left(y_{0}\right)$. De fato, basta tomar $y_{0}=\lambda^{-1} x_{0}$, para $\lambda \in S_{\mathbb{K}}$ satisfazendo a $f\left(x_{0}\right)=\lambda\left|f\left(x_{0}\right)\right|$. Notemos ainda que dado $f \in X^{*}$, a desigualdade $\operatorname{Re} f(x) \leq|f(x)|$, para todo $x \in B_{X}$, implica que $\sup \left\{\operatorname{Re} f(x): x \in B_{X}\right\}$ existe.

Proposição 2.1.3. Se $X$ é um espaço normado e $f \in X^{*}$, então $\|f\|=\sup \left\{\operatorname{Re} f(x): x \in B_{X}\right\}$.

Demonstração. Como $\operatorname{Re} f(x) \leq|f(x)|$ para todo $x \in B_{X}$, temos

$$
\sup \left\{\operatorname{Re} f(x): x \in B_{X}\right\} \leq \sup \left\{|f(x)|: x \in B_{X}\right\}
$$

e pela discussão acima, dado $x_{0} \in B_{X}$, existe $y_{0} \in B_{X}$ tal que $\left|f\left(x_{0}\right)\right|=\operatorname{Re} f\left(y_{0}\right)$, o que nos leva a concluir que

$$
\sup \left\{\operatorname{Re} f(x): x \in B_{X}\right\}=\sup \left\{|f(x)|: x \in B_{X}\right\}
$$

Notemos que a proposição acima também implica que $\|f\|=\sup \left\{|\operatorname{Re} f(x)|: x \in B_{X}\right\}$, já que $\operatorname{Re} f(x) \leq|\operatorname{Re} f(x)| \leq|f(x)|$ para todo $x \in B_{X}$.

Corolário 2.1.4. Sejam $X$ um espaço normado e $f \in X^{*} \backslash\{0\}$. Então $f$ atinge sua norma se, $e$ somente se, $f$ suporta $B_{X}$. 
Demonstração. $(\Rightarrow)$ Suponhamos que $f$ atinja sua norma. Então existe $x_{0} \in B_{X}$ tal que $\left|f\left(x_{0}\right)\right|=$ $\|f\|$. Dessa forma, como já discutido, existe $y_{0} \in B_{X}$ satisfazendo a $y_{0}=\rho x_{0}$ para algum $\rho \in S_{\mathbb{K}}$ tal que Re $f\left(y_{0}\right)=\left|f\left(x_{0}\right)\right|=|| f||$, e pela Proposição 2.1.3,

$$
\sup \left\{\operatorname{Re} f(x): x \in B_{X}\right\}=\|f\|=\left|f\left(x_{0}\right)\right|=\operatorname{Re} f\left(y_{0}\right) .
$$

Como $f \neq 0$ por hipótese, concluímos que $f$ suporta $B_{X}$ em $y_{0}$.

$(\Leftarrow)$ Suponhamos agora que $f$ suporta $B_{X}$ em $x_{0} \in B_{X}$, ou seja $\operatorname{Re} f\left(x_{0}\right)=\sup \{\operatorname{Re} f(x): x \in$ $\left.B_{X}\right\}$. Pela Proposição 2.1.3, $\|f\|=\sup \left\{\operatorname{Re} f(x): x \in B_{X}\right\}=\operatorname{Re} f\left(x_{0}\right)$. Logo,

$$
|| f|| \geq\left|f\left(x_{0}\right)\right| \geq \operatorname{Re} f\left(x_{0}\right)=\|f\|,
$$

o que implica $\left|f\left(x_{0}\right)\right|=\|f\|$, isto é, $f$ atinge a sua norma no ponto $x_{0} \in B_{X}$.

Observação 2.1.5. Notemos que da demonstração acima podemos ainda concluir um pouco mais:

- Se $f \in X^{*} \backslash\{0\}$ atinge sua norma em $x_{0} \in B_{X}$, então $f$ suporta $B_{X}$ em $\rho x_{0}$, para algum $\rho \in S_{\mathbb{K}}$.

- Se $f \in X^{*} \backslash\{0\}$ suporta $B_{X}$ em $x_{0} \in B_{X}$, então $f$ atinge sua norma em $x_{0}$.

A Proposição abaixo mostra que pontos suporte de um subconjunto sempre estarão na sua fronteira.

Proposição 2.1.6. Se $X$ é um espaço normado, $A \subset X$ e $x_{0}$ é ponto suporte para $A$, então $x_{0} \in \partial A$.

Demonstração. Como $x_{0}$ é ponto suporte para $A$, temos que $x_{0} \in A$. Assim, basta mostrar que $x_{0} \in A^{\complement} \cap B\left(x_{0} ; r\right)$ para todo $r>0$.

Consideremos então $r \in \mathbb{R}, r>0$. Por hipótese, existe $f \in X^{*} \backslash\{0\}$ tal que

$$
\operatorname{Re} f\left(x_{0}\right)=\sup \{\operatorname{Re} f(x): x \in A\} .
$$

Agora, como $f \neq 0$, deve existir $0 \neq v \in X$ tal que $f(v)=1$. O ponto $\left(x_{0}+\frac{r}{2\|v\|} v\right) \in B\left(x_{0} ; r\right)$ satisfaz a

$$
\begin{aligned}
\operatorname{Re} f\left(x_{0}+\frac{r}{2\|v\|} v\right) & =\operatorname{Re} f\left(x_{0}\right)+\frac{r}{2\|v\|} \operatorname{Re} f(v) \\
& =\operatorname{Re} f\left(x_{0}\right)+\frac{r}{2\|v\|} \\
& >\operatorname{Re} f\left(x_{0}\right) .
\end{aligned}
$$

Portanto, tendo em vista (2.1.1), concluímos que $\left(x_{0}+\frac{r}{2\|v\|} v\right) \in A^{\complement} \cap B\left(x_{0} ; r\right)$, o que implica que $x_{0} \in \partial A$, já que $r>0$ era arbitrário.

Corolário 2.1.7. Se $X$ é um espaço normado e $f \in X^{*} \backslash\{0\}$ atinge sua norma em $x_{0} \in B_{X}$, então $x_{0} \in S_{X}$.

Demonstração. Pela Observação 2.1.5, existe $\rho \in S_{\mathbb{K}}$ tal que $f$ suporta $B_{X}$ em $\rho x_{0}$, e pela Proposição 2.1.6, $\rho x_{0} \in \partial B_{X}=S_{X}$. Portanto, $x_{0} \in S_{X}$. 
Para finalizar, faremos uma observação que ajudará na demonstração de alguns resultados das seções seguintes.

Observação 2.1.8. Seja $X$ um espaço normado sobre $\mathbb{C}$. Muitas vezes é de interesse estudar o espaço vetorial obtido ao restringir a operação de multiplicação de escalares em $\mathbb{C}$ por elementos de $X$ ao corpo $\mathbb{R}$, mantendo a operação de soma. A norma de $X$ claramente também será uma norma neste novo espaço. Denotaremos tal espaço normado por $X_{\mathbb{R}}$. Como $X$ e $X_{\mathbb{R}}$ têm a mesma norma, vale que $B_{X}=B_{X_{\mathbb{R}}}, S_{X}=S_{X_{\mathbb{R}}}$ e que os espaços têm a mesma topologia. Em particular, os conjuntos abertos, fechados e limitados de ambos os espaços são os mesmos, assim como os interiores, fechos e fronteiras de subconjuntos. Vale ainda que a aplicação

$$
\begin{aligned}
R: X^{*} & \rightarrow X_{\mathbb{R}}^{*} \\
f & \mapsto \operatorname{Re} f
\end{aligned}
$$

define uma bijeção que preserva normas. A seguir mostraremos isso.

Primeiro observemos que dado $f \in X^{*}$, vale que $\|f\|=\sup \left\{|\operatorname{Re} f(x)|: x \in B_{X_{\mathbb{R}}}\right\}$, pela Proposição 2.1.3. Então de fato $\operatorname{Re} f \in X_{\mathbb{R}}^{*}$ e $R$ preserva normas. A injetividade segue pois se $f, g \in$ $X^{*}$ são tais que $\operatorname{Re} f=\operatorname{Re} g$, então para cada $x_{0} \in X$ temos $\operatorname{Re} f\left(x_{0}\right)=\operatorname{Re} g\left(x_{0}\right)$ e $\operatorname{Re} f\left(i x_{0}\right)=$ $\operatorname{Re} g\left(i x_{0}\right)$, isto é,

$$
-\operatorname{Im}\left(f\left(x_{0}\right)\right)=\operatorname{Re}\left(i f\left(x_{0}\right)\right)=\operatorname{Re}\left(i g\left(x_{0}\right)\right)=-\operatorname{Im}\left(g\left(x_{0}\right)\right),
$$

o que implica que $f\left(x_{0}\right)=g\left(x_{0}\right)$, e como $x_{0} \in X$ era arbitrário, $f=g$. Por fim, para mostrar a sobrejetividade, dado $G \in X_{\mathbb{R}}^{*}$, definamos $g: X \rightarrow \mathbb{C}$ por $g(x)=G(x)-i G(i x)$, para cada $x \in X$. Temos que $g$ é linear, pois se $x, y \in X$ e $z=a+b i, \operatorname{com} a, b \in \mathbb{R}$,

$$
\begin{aligned}
g(x+z y) & =g(x+a y+i b y) \\
& =G(x+a y+i b y)-i G(i x+i a y-b y) \\
& =G(x)+a G(y)+b G(i y)-i G(i x)-i a G(i y)+i b G(y) \\
& =g(x)+a g(y)+i b(G(y)-i G(i y)) \\
& =g(x)+z g(y) .
\end{aligned}
$$

Além disso, $|g(x)| \leq|G(x)|+|G(i x)| \leq 2|| G\left\|_{X_{\mathbb{R}}^{*}} \cdot\right\| x \|$ para todo $x \in X$, e então $g \in X^{*}$. Como $\operatorname{Re} g=G$, está mostrada a sobrejetividade de $R$.

É claro que se $X$ for um espaço normado sobre $\mathbb{R}$, restringir a multiplicação por escalares a $\mathbb{R}$ não produz um espaço novo, e assim $X_{\mathbb{R}}=X$.

\subsection{Teorema de Bishop-Phelps}

Esta seção tem como principal objetivo a demonstração do Teorema de Bishop-Phelps, que afirma que para todo espaço de Banach $X$, vale que $\mathcal{N} \mathcal{A}_{X}$ é denso em $X^{*}$. Este resultado foi demonstrado pela primeira vez no artigo [BP61], de 1961. Dois anos depois, em [BP63], Bishop e Phelps generalizaram e simplificaram os métodos utilizados no artigo anterior, obtendo uma demonstração mais elegante do Teorema. Basearemo-nos aqui em [Meg98] e nessa generalização, que permite obter outros resultados interessantes. 
Definição 2.2.1. Um espaço normado $X$ é dito subreflexivo se $\mathcal{N} \mathcal{A}_{X}$ é denso em $X^{*}$.

Existem espaços normados incompletos subreflexivos e não-subreflexivos, como veremos a seguir.

Proposição 2.2.2. Todo espaço com produto interno é subreflexivo.

Demonstração. Seja $Y$ um espaço com produto interno. Conforme a Proposição 1.1.8, existe um espaço de Hilbert $H$ e uma imersão isométrica $J: Y \rightarrow H$ tal que $J(Y)$ é denso em $H$. Vamos identificar aqui os espaços $J(Y)$ e $Y$ e dizer simplesmente que $Y \subset H$ e $Y$ é denso em $H$.

Considere a aplicação $Q: H \rightarrow H^{*}$ dada por $Q(v)=f_{v}$. Pelo Teorema de Riesz-Fréchet e a discussão que o antecede, $Q$ é bijeção, preserva normas e vale ainda que $Q\left(v_{1}+v_{2}\right)=Q\left(v_{1}\right)+Q\left(v_{2}\right)$ para todos $v_{1}, v_{2} \in H$. Logo, $Q(Y)$ é denso em $H^{*}$. Como $Y$ é denso em $H$, da Proposição 1.1 .12 temos que a aplicação $P: H^{*} \rightarrow Y^{*}$ dada por $P(f)=\left.f\right|_{Y}$ é uma isometria, donde concluímos que $P(Q(Y))$ é denso em $Y^{*}$. Agora basta mostrar que todo funcional em $P(Q(Y))$ atinge a sua norma. Para isso, observemos que dado $f \in Q(Y)$, com $f=Q(u)=f_{u}, u \in Y, f$ atinge sua norma em $\frac{u}{\|u\|} \in B_{Y}$ se $u \neq 0$ e em $0 \in B_{Y}$ se $u=0$. Ou seja, para cada $f \in Q(Y)$ existe $v \in B_{Y}$ tal que $\|f\|=|f(v)|$, e assim

$$
|f|_{Y}(v)|=| f(v)\left|=\|f\|=\|P(f)\|=\left\|\left.f\right|_{Y}\right\| .\right.
$$

Portanto, $P(f)$ atinge sua norma para todo $f \in Q(Y)$ e, pela densidade de $P(Q(Y))$ em $Y^{*}$, segue que $Y$ é subreflexivo.

Com a Proposição 2.2.2 fica fácil construir exemplos de espaços normados incompletos subreflexivos. Basta considerar qualquer espaço com produto interno que não é espaço de Hilbert, como por exemplo $\left(c_{00},\|.\|_{2}\right)$, em que $c_{00}$ denota o espaço vetorial das sequências quase-nulas de escalares em $\mathbb{K}$.

Em [Phe57] foi dado um exemplo de espaço normado incompleto que não é subreflexivo. Este exemplo não será apresentado aqui, pois faz uso de alguns resultados que fogem do escopo deste trabalho.

A seguir introduziremos os conceitos de cone e ponto suporte cônico, noções importantes para a demonstração do Teorema de Bishop-Phelps que apresentaremos.

Definição 2.2.3. Seja $V$ um espaço vetorial. Um subconjunto não-vazio $K \subset V$ é dito um cone em $V$ se satisfizer as seguintes condições:

(i) $K$ é convexo;

(ii) $t K \subset K$ para todo $t \in \mathbb{R}_{\geq 0}$;

(iii) $K \cap(-K)=\{0\}$.

Observaçâo 2.2.4. É importante ressaltar que a nomenclatura cone é usada para se referir a diferentes conceitos, dependendo da referência. Por exemplo, Bishop e Phelps em [BP63] chamam de cone convexo um subconjunto que satisfaz apenas as condições (i) e (ii) dadas acima. Usamos aqui a notação de [Meg98].

Notemos que se $V$ é um espaço vetorial e $K \subset V$ é um cone em $V$, o item (ii) da Definição 2.2.3 para $t=0$ implica que $0 K=\{0\} \subset K$, ou seja, $0 \in K$. 
O nome cone não é por acaso. De fato, os sólidos que conhecemos como cones (infinitamente estendidos) em $\mathbb{R}^{3}$, com vértice na origem, também são cones segundo a Definição 2.2.3, como exemplificamos abaixo.

Exemplo 2.2.5. Considere, para $a, b \in \mathbb{R}, a, b>0$, o cone sólido $C$ infinitamente estendido em $\mathbb{R}^{3}$, definido por:

$$
C=\left\{(x, y, z) \in \mathbb{R}^{3}: \sqrt{\left(\frac{x}{a}\right)^{2}+\left(\frac{y}{b}\right)^{2}} \leq z\right\} .
$$

Afirmamos que $C$ é um cone (segundo a Definição 2.2.3) em $\mathbb{R}^{3}$.

Como $(0,0,0) \in C, C \neq \emptyset$. Mostraremos (i), (ii) e (iii):

(i) Sejam $\left(x_{1}, y_{1}, z_{1}\right),\left(x_{2}, y_{2}, z_{2}\right) \in C$ e $\lambda \in[0,1]$. Vale que:

$$
\begin{aligned}
& \sqrt{\left(\frac{x_{1}}{a}\right)^{2}+\left(\frac{y_{1}}{b}\right)^{2}} \leq z_{1} ; \\
& \sqrt{\left(\frac{x_{2}}{a}\right)^{2}+\left(\frac{y_{2}}{b}\right)^{2}} \leq z_{2} .
\end{aligned}
$$

Vamos mostrar que $\left(\lambda\left(x_{1}, y_{1}, z_{1}\right)+(1-\lambda)\left(x_{2}, y_{2}, z_{2}\right)\right) \in C$, ou seja,

$$
\sqrt{\left(\frac{\lambda x_{1}+(1-\lambda) x_{2}}{a}\right)^{2}+\left(\frac{\lambda y_{1}+(1-\lambda) y_{2}}{b}\right)^{2}} \leq \lambda z_{1}+(1-\lambda) z_{2} .
$$

Para isso, definamos $\gamma_{1}$ e $\gamma_{2} \in \mathbb{R}^{2}$ por:

$$
\begin{gathered}
\gamma_{1} \doteq \lambda\left(\frac{x_{1}}{a}, \frac{y_{1}}{b}\right) ; \\
\gamma_{2} \doteq(1-\lambda)\left(\frac{x_{2}}{a}, \frac{y_{2}}{b}\right) .
\end{gathered}
$$

Pela Desigualdade Triangular da norma $\|.\|_{2}$ em $\mathbb{R}^{2},\left\|\gamma_{1}+\gamma_{2}\right\|_{2} \leq\left\|\gamma_{1}\right\|_{2}+\left\|\gamma_{2}\right\|_{2}$, ou seja,

$$
\begin{aligned}
\left\|\gamma_{1}+\gamma_{2}\right\|_{2} & =\sqrt{\left(\frac{\lambda x_{1}+(1-\lambda) x_{2}}{a}\right)^{2}+\left(\frac{\lambda y_{1}+(1-\lambda) y_{2}}{b}\right)^{2}} \\
& \leq\left\|\gamma_{1}\right\|_{2}+\left\|\gamma_{2}\right\|_{2} \\
& =\lambda \sqrt{\left(\frac{x_{1}}{a}\right)^{2}+\left(\frac{y_{1}}{b}\right)^{2}}+(1-\lambda) \sqrt{\left(\frac{x_{2}}{a}\right)^{2}+\left(\frac{y_{2}}{b}\right)^{2}} \\
& \left(\begin{array}{c}
2.2 .1) \\
\leq \lambda z_{1}+(1-\lambda) z_{2} .
\end{array}\right.
\end{aligned}
$$

como queríamos.

(ii) Se $t=0$, é claro que $t C=\{(0,0,0)\} \subset C$. Considere então $t>0$ e $(x, y, z) \in t C$, ou seja, $\left(\frac{x}{t}, \frac{y}{t}, \frac{z}{t}\right) \in C$. Isso implica que

$$
\sqrt{\left(\frac{x}{t a}\right)^{2}+\left(\frac{y}{t b}\right)^{2}} \leq \frac{z}{t}
$$


Multiplicando a desigualdade acima por $t$, vem

$$
\sqrt{\left(\frac{x}{a}\right)^{2}+\left(\frac{y}{b}\right)^{2}} \leq z,
$$

e portanto $(x, y, z) \in C$, donde concluímos que $t C \subset C$.

(iii) Notemos que se $\pm(x, y, z) \in C$, então em particular $\pm z \geq 0$, o que implica que $z=0$. Nesse caso, $\sqrt{\left(\frac{x}{a}\right)^{2}+\left(\frac{y}{b}\right)^{2}} \leq 0$, o que só ocorre se $x=y=0$. Então $(x, y, z)=(0,0,0)$, como queríamos mostrar.

Logo, $C$ é um cone.

Outro exemplo simples de cone em $\mathbb{R}^{n}$ são as semi-retas (fechadas) iniciando na origem.

Em seguida daremos uma definição alternativa para cones, que nos ajudará em alguns dos resultados seguintes.

Proposição 2.2.6. Sejam $V$ um espaço vetorial $e \emptyset \neq K \subset V$. As seguintes afirmações são equivalentes:

(a) $K$ é um cone em $V$.

(b) $K \cap(-K)=\{0\}$ e dados $x, y \in K$ e $s, t \in \mathbb{R}$ com $s, t \geq 0$, vale que sx $+t y \in K$.

Demonstração. (a) $\Rightarrow$ (b) Como $K$ é cone em $V$, pelo item (iii) da Definição 2.2.3, vale automaticamente que $K \cap(-K)=\{0\}$. Agora sejam $x, y \in K$ e $s, t \in \mathbb{R}$ com $s, t \geq 0$. Pelo item (ii) da Definição 2.2.3, $2 s x \in K$ e $2 t y \in K$, e pela convexidade de $K$ (item (i)),

$$
\frac{1}{2} 2 s x+\frac{1}{2} 2 t y=s x+t y \in K .
$$

(b) $\Rightarrow$ (a) Para mostrar o item (i) da Definição 2.2.3, basta observar que para quaisquer $x, y \in K$ e $\lambda \in[0,1]$, escolhendo $t=\lambda$ e $s=(1-\lambda)$ na hipótese do item (b), obtemos que $\lambda x+(1-\lambda) y \in K$. Para mostrar (ii) observemos que dado $t \geq 0$ e $s=0$, pela mesma hipótese, $t x+0 x=t x \in K$ para todo $x \in K$. O item (iii) é direto.

Note que se tivermos $x_{0} \in A \subset X$, com $X$ espaço normado, e $K$ um cone em $X$, sempre valerá que $x_{0} \in A \cap\left(x_{0}+K\right)$, já que $0 \in K$. O caso em que também vale que $A \cap\left(x_{0}+K\right) \subset\left\{x_{0}\right\}$, para $K$ de interior não-vazio, é interessante para os resultados seguintes. A definição abaixo trata disso.

Definição 2.2.7. Sejam $X$ um espaço normado, $A \subset X$ e $x_{0} \in A$. Dizemos que $x_{0}$ é um ponto suporte cônico para $A$ se existir um cone $K$ de interior não-vazio em $X$ tal que $A \cap\left(x_{0}+K\right)=\left\{x_{0}\right\}$.

Nas condições da definição acima, também dizemos que $K$ é um cone suporte para $A$, ou que $\left(x_{0}+K\right)$ suporta $A$ em $x_{0}$.

Seguem agora mais definições e resultados úteis para este capítulo.

Definição 2.2.8. Sejam $X$ um espaço normado, $f \in S_{X^{*}}$ e $t>1$. Definimos:

$$
K(f, t) \doteq\{x \in X:\|x\| \leq \operatorname{Re} f(t x)\} .
$$

Observemos que para quaisquer $f \in S_{X^{*}}$ e $t>1$ vale que $0 \in K(f, t)$, e portanto sempre teremos $K(f, t) \neq \emptyset$. Neste ponto também é importante ressaltar que a existência de um $f \in S_{X^{*}}$ já implica automaticamente que $X \neq\{0\}$, fato que usaremos em algumas das demonstrações seguintes. 
Proposição 2.2.9. Sejam $X$ um espaço normado, $f \in S_{X^{*}}$ e $t>1$. Então $K(f, t)$ é um cone fechado com interior não-vazio.

Demonstração. Já sabemos que $K(f, t) \neq \emptyset$. Como a aplicação $\operatorname{Re} f: X \ni x \mapsto \operatorname{Re} f(x) \in \mathbb{R}$ é contínua, segue que $F: X \rightarrow \mathbb{R}$ dada por $F(x)=\|x\|-t \operatorname{Re} f(x)$, para cada $x \in X$, é contínua. Logo, $F^{-1}((-\infty, 0])=K(f, t)$ é fechado em $X$.

Observemos agora que

$$
F^{-1}((-\infty, 0))=\{x \in X:\|x\|<\operatorname{Re} f(t x)\} \subset K(f, t)
$$

é aberto em $X$. Mostraremos em seguida que $F^{-1}((-\infty, 0)) \neq \emptyset$, o que nos permitirá concluir que $K(f, t)$ tem interior não-vazio. De fato, como $f \in S_{X^{*}}$, pela Proposição 2.1.3, $\sup \{\operatorname{Re} f(x): x \in$ $\left.B_{X}\right\}=1$, e então existe $x_{0} \in B_{X}$ tal que $\operatorname{Re} f\left(x_{0}\right)>\frac{1}{t}$, donde segue que $\operatorname{Re} f\left(t x_{0}\right)>1=\left\|x_{0}\right\|$, ou seja, $x_{0} \in F^{-1}((-\infty, 0))$. Então $K(f, t)^{\circ} \neq \emptyset$.

Resta mostrar que $K(f, t)$ é um cone. Usaremos para isso a caracterização de cone dada pelo item $(b)$ da Proposição 2.2.6. Já sabemos que $0 \in K(f, t) \cap(-K(f, t))$. Por outro lado, se $z \in$ $K(f, t) \cap(-K(f, t))$, ou seja, $\pm z \in K(f, t)$, então $\| \pm z\| \leq \operatorname{Re} f( \pm t z)$, donde concluímos que $z=0$. Assim, $K(f, t) \cap(-K(f, t))=\{0\}$. Considere agora $x, y \in K(f, t)$ e $s_{1}, s_{2} \in \mathbb{R}$, com $s_{1}, s_{2} \geq 0$. Por definição, $\|x\| \leq \operatorname{Re} f(t x)$ e $\|y\| \leq \operatorname{Re} f(t y)$, o que implica

$$
\begin{aligned}
& s_{1}\|x\|=\left\|s_{1} x\right\| \leq \operatorname{Re} f\left(t s_{1} x\right) ; \\
& s_{2}\|y\|=\left\|s_{2} y\right\| \leq \operatorname{Re} f\left(t s_{2} y\right) .
\end{aligned}
$$

Somando as equações acima e usando a desigualdade triangular, obtemos

$$
\left\|s_{1} x+s_{2} y\right\| \leq\left\|s_{1} x\right\|+\left\|s_{2} y\right\| \leq \operatorname{Re} f\left(t\left(s_{1} x+s_{2} y\right)\right) .
$$

Então $\left(s_{1} x+s_{2} y\right) \in K(f, t)$ e, pela Proposição 2.2.6, $K(f, t)$ é um cone.

Observação 2.2.10. Notemos que embora $K(f, t)^{\circ} \neq \emptyset$ sempre que $f \in S_{X^{*}}$ e $t>1$, podemos garantir que $0 \notin K(f, t)^{\circ}$. De fato, caso $0 \in K(f, t)^{\circ}$, teríamos que $B(0 ; \epsilon) \subset K(f, t)$ para algum $\epsilon>0$. Como $X \neq\{0\}$, deve existir $x \in B(0 ; \epsilon) \backslash\{0\}$, e nesse caso $\pm x \in K(f, t)$, absurdo, pois $K(f, t)$ é um cone, como acabamos de provar.

Lema 2.2.11. Sejam $X$ um espaço normado, $A \subset X$, com $A$ completo, $x \in A, t>1$ e $f \in S_{X^{*}}$ tal que Re $f(A)$ é limitado. Então existe $x_{0} \in A$ tal que $x_{0} \in(x+K(f, t))$ e $\left(x_{0}+K(f, t)\right)$ suporta A em $x_{0}$.

Demonstração. Definamos $B \doteq A \cap(x+K(f, t)) \subset A$. Como $x \in B$, temos que $B \neq \emptyset$. Pela Proposição 2.2.9, $K(f, t)$ é fechado, e assim $(x+K(f, t))$ é fechado. Como $A$ é completo, em particular também é fechado. Logo, $B$ é subconjunto fechado de um conjunto completo, o que implica que $B$ é completo. Definamos em $B$ a relação $\preceq$ dada por:

$$
z \preceq w \Leftrightarrow(w-z) \in K(f, t), \text { para todos } z, w \in B .
$$

Afirmamos que a relação $\preceq$ é uma ordem parcial para $B$. De fato, se $b \in B$, então $(b-b)=0 \in$ $K(f, t)$. Ou seja, $b \preceq b$ para todo $b \in B$, e assim a relação é reflexiva. Para mostrar que $\preceq$ é 
antissimétrica, observemos que se $a, b \in B$ são tais que $a \preceq b$ e $b \preceq a$, então $\pm(a-b) \in K(f, t)$. Pela Proposição 2.2.9, $K(f, t)$ é cone, e então $a-b=0$, ou seja, $a=b$. Sejam agora $a, b, c \in B$ tais que $a \preceq b$ e $b \preceq c$. Então $(b-a) \in K(f, t)$ e $(c-b) \in K(f, t)$. Novamente usando que $K(f, t)$ é cone, pela Proposição 2.2.6 $(b-a)+(c-b)=(c-a) \in K(f, t)$, e então $a \preceq c$. Mostramos assim que $(B, \preceq)$ é um conjunto parcialmente ordenado. Provaremos a seguir que $B$ possui um elemento maximal segundo a ordem definida. Para isso, será mostrado que toda cadeia não-vazia em $B$ possui cota superior, e a conclusão virá do Lema de Zorn.

Seja $\mathcal{C}$ uma cadeia não-vazia em $(B, \preceq)$. Como $\preceq$ é uma ordem parcial, em particular $\mathcal{C}$ é um conjunto dirigido por tal relação. Considere a rede $\left(r_{x}\right)_{x \in \mathcal{C}}$ em $\mathbb{R}$ dada por $r_{x}=\operatorname{Re} f(x)$, para cada $x \in \mathcal{C}$. Notemos que se $z, y \in \mathcal{C}$ são tais que $z \preceq y$, então $(y-z) \in K(f, t)$, ou seja, $0 \leq\|y-z\| \leq \operatorname{Re} f(t(y-z))$, e em consequência $\operatorname{Re} f(z) \leq \operatorname{Re} f(y)$. Assim, vale que a rede é crescente. Como $\mathcal{C} \subset A$, por hipótese existe $M \in \mathbb{R}_{\geq 0}$ tal que $\operatorname{Re} f(x) \leq M$ para todo $x \in \mathcal{C}$, e dessa forma o conjunto $\{\operatorname{Re} f(x): x \in \mathcal{C}\}$ admite um supremo $M_{0} \in \mathbb{R}_{\geq 0}$. Portanto, a rede $\left(r_{x}\right)_{x \in \mathcal{C}}$ em $\mathbb{R}$ também é limitada superiormente, e então convergirá para $M_{0}$. Em particular, será uma rede de Cauchy. Seja agora $\left(i_{x}\right)_{x \in \mathcal{C}}$ a rede "identidade"em $B$, isto é, $i_{x}=x$ para todo $x \in \mathcal{C}$. Vamos mostrar que $\left(i_{x}\right)_{x \in \mathcal{C}}$ é rede de Cauchy em $B$. Para isso, tome $\epsilon>0$. Como $\left(r_{x}\right)_{x \in \mathcal{C}}$ é rede de Cauchy em $\mathbb{R}$, existe $x_{0} \in \mathcal{C}$ tal que

$$
x_{1}, x_{2} \in \mathcal{C}, x_{0} \preceq x_{1}, x_{2} \Rightarrow\left|\operatorname{Re} f\left(x_{1}\right)-\operatorname{Re} f\left(x_{2}\right)\right|<\frac{\epsilon}{t}
$$

Consideremos então $x_{1}, x_{2} \in \mathcal{C}$ satisfazendo a $x_{0} \preceq x_{1}, x_{2}$. Como $\mathcal{C}$ é cadeia, podemos assumir sem perda de generalidade que $x_{2} \preceq x_{1}$, então por definição $\left\|x_{1}-x_{2}\right\| \leq \operatorname{Re} f\left(t\left(x_{1}-x_{2}\right)\right)<\epsilon$, e assim concluímos que a rede $\left(i_{x}\right)_{x \in \mathcal{C}}$ é de Cauchy. Como $B$ é completo, existe $u \in B$ tal que $i_{x} \rightarrow u$. Mostraremos que $u$ é cota superior para $\mathcal{C}$. Com efeito, considere $y \in \mathcal{C}$. Para todo $x \in \mathcal{C}$ tal que $y \preceq x$, vale que $(x-y)=\left(i_{x}-y\right) \in K(f, t)$. Como $K(f, t)$ é fechado, $\left(i_{x}-y\right) \rightarrow(u-y) \in K(f, t)$. Ou seja, $y \preceq u$ para todo $y \in \mathcal{C}$. Portanto, toda cadeia não-vazia em $B$ tem cota superior e assim, pelo Lema de Zorn, existe $x_{0} \in B$ elemento maximal para $B$. Pela definição de $B$, temos que $x_{0} \in(x+K(f, t))$ e $x_{0} \in A$. Resta mostrar que $\left(x_{0}+K(f, t)\right)$ suporta $A$ em $x_{0}$, o que faremos em seguida.

Observemos que pela Proposição 2.2.9 já sabemos que $K(f, t)$ é um cone de interior não-vazio. Seja $y \in A \cap\left(x_{0}+K(f, t)\right)$. Da Proposição 2.2.6, podemos concluir que $K(f, t)+K(f, t)=K(f, t)$. Então

$$
x_{0}+K(f, t) \subset(x+K(f, t))+K(f, t)=x+(K(f, t)+K(f, t))=x+K(f, t),
$$

donde vem que $y \in A \cap(x+K(f, t))=B$ e $y \in\left(x_{0}+K(f, t)\right)$. Portanto, $x_{0} \preceq y$, o que implica $x_{0}=y$, pela maximalidade de $x_{0}$. A conclusão é que $A \cap\left(x_{0}+K(f, t)\right)=\left\{x_{0}\right\}$, ou seja, $\left(x_{0}+K(f, t)\right)$ suporta $A$ em $x_{0}$.

Sejam $X$ um espaço de Banach e $C \subset X$. A pergunta-chave para a demonstração do Teorema de Bishop-Phelps que apresentaremos aqui é a seguinte:

\section{$E$ verdade que o conjunto dos funcionais que suportam $C$ é denso em $X^{*}$ ?}

Buscaremos no que segue condições sobre $C$ para que essa pergunta seja afirmativamente respondida. Notemos que se a resposta for positiva quando $C=B_{X}$, pelo Corolário 2.1.4, o Teorema de 
Bishop-Phelps seguirá. Observemos ainda que se $C=\emptyset$ ou $C=X$ não existirão funcionais suporte para $C$. Entretanto, não podemos garantir uma resposta positiva para a pergunta em geral nem se $C$ for fechado, convexo, $C \neq \emptyset$ e $C \neq X$, como mostra o exemplo abaixo.

Exemplo 2.2.12. Considere o espaço de Banach $X=\ell_{1}^{2}$, sobre $\mathbb{R}$, e seja $C=\mathbb{R} \times\{0\}$.

É claro que $C$ é fechado e convexo. Vamos mostrar que $D \doteq\left\{f \in X^{*}: f\right.$ suporta $\left.C\right\}$ não é denso em $X^{*}$. Afirmamos que $D=\left\{f \in X^{*}: f(x, y)=b y, b \in \mathbb{R} \backslash\{0\}\right\}$. De fato, dado $g \in D$, temos que $g(x, y)=a x+b y$ para certos $a, b \in \mathbb{R}$. Existe $\left(x_{0}, 0\right) \in C$ tal que $g\left(x_{0}, 0\right)=\sup \{g(x, y)$ : $(x, y) \in C\}=\sup \{g(x, 0): x \in \mathbb{R}\}$. Logo, $g\left(x_{0}, 0\right)=a x_{0} \geq a x$ para todo $x \in \mathbb{R}$, o que implica que $a\left(x_{0}-x\right) \geq 0$ para todo $x \in \mathbb{R}$. Escolhendo $x=x_{0}+a$, obtemos $-a^{2} \geq 0$, então $a=0$. Como $g \neq 0$, temos que $b \neq 0$, e assim $g \in\left\{f \in X^{*}: f(x, y)=b y, b \in \mathbb{R} \backslash\{0\}\right\}$. Seja agora $g \in X^{*}$ tal que $g(x, y)=b y, b \neq 0$. Então $g(x, 0)=0$ para todo $(x, 0) \in C$, e assim $g \in D$.

Resta provar que $D$ não é denso em $X^{*}$. Para isso, considere $f \in X^{*}$ dado por $f(x, y)=x$ para todo $(x, y) \in X$. Se $g \in D$, então $g(x, y)=b y$ para certo $b \neq 0$. Assim,

$$
|| g-f|| \geq|g(1,0)-f(1,0)|=1,
$$

e portanto todos os elementos de $D$ distam de $f$ de mais de 1 , o que implica que $D$ não é denso em $X^{*}$.

Entretanto, se $X \neq\{0\}$, podemos garantir que o conjunto dos funcionais que suportam $C$ é denso em $X^{*}$ se $C$ for não-vazio, fechado, convexo e limitado. Para mostrar isso, precisamos de alguns resultados auxiliares, que serão feitos a seguir.

Lema 2.2.13. Sejam $X$ um espaço normado sobre $\mathbb{R}, f_{1}, f_{2} \in S_{X^{*}}$ e $\epsilon>0$. Se $f_{2}\left(\operatorname{Ker} f_{1} \cap B_{X}\right) \subset$ $[-\epsilon / 2, \epsilon / 2]$, então

$$
\left\|f_{1}-f_{2}\right\| \leq \epsilon \text { ou }\left\|f_{1}+f_{2}\right\| \leq \epsilon .
$$

Demonstração. Seja $\left.F \doteq f_{2}\right|_{\operatorname{Ker} f_{1}} \in\left(\operatorname{Ker} f_{1}\right)^{*}$. Notemos que se $y \in B_{\operatorname{Ker} f_{1}}=\operatorname{Ker} f_{1} \cap B_{X}$, então $|F(y)|=\left|f_{2}(y)\right| \leq \frac{\epsilon}{2}$. Logo, $\|F\|_{\left(\operatorname{Ker} f_{1}\right)^{*}} \leq \frac{\epsilon}{2}$. Pelo Teorema de Hahn-Banach, existe $f \in X^{*}$ tal que $F=\left.f\right|_{\operatorname{Ker} f_{1}}$ e $\|f\|=\|F\|_{\left(\operatorname{Ker} f_{1}\right)^{*}} \leq \frac{\epsilon}{2}$. Em particular, $f_{2}(y)=f(y)$ para todo $y \in \operatorname{Ker} f_{1}$, e então $\operatorname{Ker} f_{1} \subset \operatorname{Ker}\left(f_{2}-f\right)$. Pelo Lema 1.1.3, existe $\alpha \in \mathbb{R}$ tal que $\left(f_{2}-f\right)=\alpha f_{1}$. Assim,

$$
|1-| \alpha||=||\left|f_{2}\right||-| \alpha||\left|f_{1}\right||| \leq\left\|f_{2}-\alpha f_{1}||=\right\| f \| \leq \frac{\epsilon}{2} .
$$

Se $\alpha \geq 0,|1-\alpha|=|1-| \alpha|| \leq \frac{\epsilon}{2}$, e então

$$
\left\|f_{2}-f_{1}\right\|=\left\|f_{2}-\alpha f_{1}+\alpha f_{1}-f_{1}\right\| \leq\|f\|+|1-\alpha| \leq \epsilon .
$$

Se $\alpha<0,|1+\alpha|=|1-| \alpha|| \leq \frac{\epsilon}{2}$, e então

$$
\left\|f_{2}+f_{1}\right\|=\left\|f_{2}-\alpha f_{1}+\alpha f_{1}+f_{1}\right\| \leq\|f\|+|1+\alpha| \leq \epsilon .
$$

Isso conclui a demonstração.

Lema 2.2.14. Sejam $X$ um espaço normado sobre $\mathbb{R}, 0<\epsilon<1, f_{1}, f_{2} \in S_{X^{*}}$ e $t>(1+2 / \epsilon)$ tais que $f_{2}$ é não-negativo em $K\left(f_{1}, t\right)$. Então $\left\|f_{2}-f_{1}\right\| \leq \epsilon$. 
Demonstração. Vamos mostrar que $f_{1}$ e $f_{2}$ satisfazem as condições do Lema 2.2.13. Primeiro observemos que, como $\left\|f_{1}\right\|=1$, existe $x_{0} \in B_{X}$ tal que

$$
f_{1}\left(x_{0}\right)>\operatorname{máx}\left\{t^{-1}\left(1+\frac{2}{\epsilon}\right), \epsilon\right\} \text {. }
$$

Assim, $\left\|x_{0}\right\|=1<\left(1+\frac{2}{\epsilon}\right)<f_{1}\left(t x_{0}\right)$, e portanto $x_{0} \in K\left(f_{1}, t\right)$. Dessa forma, por hipótese, $f_{2}\left(x_{0}\right) \geq 0$.

Agora considere $x \in B_{X} \cap \operatorname{Ker} f_{1}$. Temos que

$$
\left\|x_{0} \pm \frac{2}{\epsilon} x\right\| \leq\left(1+\frac{2}{\epsilon}\right) \leq t f_{1}\left(x_{0}\right)=t f_{1}\left(x_{0} \pm \frac{2}{\epsilon} x\right)
$$

e portanto $x_{0} \pm(2 / \epsilon) x \in K\left(f_{1}, t\right)$, o que implica que $f_{2}\left(x_{0} \pm(2 / \epsilon) x\right) \geq 0$, ou seja,

$$
f_{2}\left(x_{0}\right) \geq \pm \frac{2}{\epsilon} f_{2}(x)
$$

Logo, $\left|\frac{2}{\epsilon} f_{2}(x)\right| \leq f_{2}\left(x_{0}\right) \leq 1$, donde vem que $\left|f_{2}(x)\right| \leq \frac{\epsilon}{2}$ para todo $x \in B_{X} \cap \operatorname{Ker} f_{1}$. Pelo Lema 2.2.13, vale que $\left\|f_{1}-f_{2}\right\| \leq \epsilon$ ou $\left\|f_{1}+f_{2}\right\| \leq \epsilon$. Mas $\left\|f_{1}+f_{2}\right\| \geq\left|\left(f_{1}+f_{2}\right)\left(x_{0}\right)\right| \geq f_{1}\left(x_{0}\right)+f_{2}\left(x_{0}\right)>\epsilon$, pois $f_{1}\left(x_{0}\right)>\epsilon$ e $f_{2}\left(x_{0}\right) \geq 0$. A conclusão é que $\left\|f_{1}-f_{2}\right\| \leq \epsilon$.

O Teorema seguinte é um importante resultado de separação demonstrado por Bishop e Phelps em [BP63].

Teorema 2.2.15. Sejam $X$ um espaço de Banach e $C, A \subset X$ subconjuntos não-vazios tais que $A$ é limitado e $C$ é fechado e convexo. Suponha que $\epsilon>0$ e que $f_{1} \in S_{X^{*}}$ seja tal que

$$
\sup \left\{\operatorname{Re} f_{1}(x): x \in C\right\}<\inf \left\{\operatorname{Re} f_{1}(x): x \in A\right\} .
$$

Então existem $f_{2} \in S_{X^{*}}$ e $x_{0} \in C$ tais que $\left\|f_{1}-f_{2}\right\| \leq \epsilon e$

$$
\operatorname{Re} f_{2}\left(x_{0}\right)=\sup \left\{\operatorname{Re} f_{2}(x): x \in C\right\}<\inf \left\{\operatorname{Re} f_{2}(x): x \in A\right\}
$$

Demonstração. Sem perda de generalidade, podemos assumir $\epsilon<1$. Seja

$$
\delta \doteq \frac{1}{2}\left(\inf \left\{\operatorname{Re} f_{1}(x): x \in A\right\}-\sup \left\{\operatorname{Re} f_{1}(x): x \in C\right\}\right)>0
$$

e $B \doteq A+\delta B_{X}$. O conjunto $B$ é limitado, pois $A$ e $B_{X}$ o são. Em particular, $\inf \left\{\operatorname{Re} f_{1}(x): x \in B\right\}$ é finito. Temos que

$$
\begin{aligned}
\inf \left\{\operatorname{Re} f_{1}(x): x \in B\right\} & =\inf \left\{\operatorname{Re} f_{1}(x): x \in A\right\}+\inf \left\{\operatorname{Re} f_{1}(x): x \in \delta B_{X}\right\} \\
& =\inf \left\{\operatorname{Re} f_{1}(x): x \in A\right\}+\delta \inf \left\{\operatorname{Re} f_{1}(x): x \in B_{X}\right\}
\end{aligned}
$$

Como $-B_{X}=B_{X}$ e $\inf \left\{\operatorname{Re} f_{1}(x): x \in B_{X}\right\}=-\sup \left\{\operatorname{Re} f_{1}(x): x \in-B_{X}\right\}$, pela Proposição 2.1.3, 
$\inf \left\{\operatorname{Re} f_{1}(x): x \in B_{X}\right\}=-\left\|f_{1}\right\|=-1 . \operatorname{Logo}, \inf \left\{\operatorname{Re} f_{1}(x): x \in B\right\}=\inf \left\{\operatorname{Re} f_{1}(x): x \in A\right\}-\delta$, isto é,

$$
\inf \left\{\operatorname{Re} f_{1}(x): x \in B\right\}=\delta+\sup \left\{\operatorname{Re} f_{1}(x): x \in C\right\} .
$$

Seja $s \doteq 1+2 / \epsilon$. Existe $z \in C$ tal que

$$
\sup \left\{\operatorname{Re} f_{1}(x): x \in C\right\}-\operatorname{Re} f_{1}(z)<\frac{\delta}{2 s}
$$

Como $B$ é limitado, $\sup \{\|y-z\|: y \in B\}$ é finito. Tome $M, t \in \mathbb{R}$ tais que

$$
M>\operatorname{máx}\left\{\frac{\delta}{2}, \sup \{\|y-z\|: y \in B\}\right\} \text { e } t \doteq \frac{2 s M}{\delta} .
$$

É claro que $t>s>1$, por construção. Temos que $X$ é espaço de Banach e $C$ é fechado em $X$, o que implica que $C$ é completo. Assim, podemos aplicar o Lema 2.2.11 para concluir que existe $x_{0} \in C$ tal que $\left(x_{0}-z\right) \in K\left(f_{1}, t\right)$ e $\left(x_{0}+K\left(f_{1}, t\right)\right)$ suporta $C$ em $x_{0}$.

Afirmamos agora que $B \subset\left(x_{0}+K\left(f_{1}, t\right)\right)$. De fato, se $y \in B$,

$$
\begin{aligned}
\left\|y-x_{0}\right\| & \leq\|y-z\|+\left\|x_{0}-z\right\| \\
& \leq M+\operatorname{Re} f_{1}\left(t\left(x_{0}-z\right)\right) \\
& =M+t\left(\operatorname{Re} f_{1}\left(x_{0}\right)-\operatorname{Re} f_{1}(z)\right) \\
& \stackrel{(2.2 .3)}{\leq} M+t\left(\frac{\delta}{2 s}\right) \\
& =\frac{\delta t}{s} \\
& <t \delta \\
& (2.2 .2) \\
& \leq t\left(\operatorname{Re} f_{1}\left(y-x_{0}\right)\right) .
\end{aligned}
$$

Portanto, $\left(y-x_{0}\right) \in K\left(f_{1}, t\right)$. Como $y \in B$ era arbitrário, $B \subset\left(x_{0}+K\left(f_{1}, t\right)\right)$.

Já temos que $\left(x_{0}+K\left(f_{1}, t\right)\right) \cap C=\left\{x_{0}\right\}$. Afirmamos que $\left(x_{0}+K\left(f_{1}, t\right)\right)^{\circ} \cap C=\emptyset$. De fato, uma vez que $\left(x_{0}+K\left(f_{1}, t\right)\right)^{\mathrm{o}}=x_{0}+K\left(f_{1}, t\right)^{\mathrm{o}}$, se tivéssemos $x_{0} \in\left(x_{0}+K\left(f_{1}, t\right)\right)^{\mathrm{o}}$, valeria que $0 \in K\left(f_{1}, t\right)^{\mathrm{o}}$, o que não pode ocorrer, pela Observação 2.2.10. Notemos ainda que pela Proposição 2.2.9, $K\left(f_{1}, t\right)^{\mathrm{o}} \neq \emptyset$ e $K\left(f_{1}, t\right)$ é convexo. Podemos então aplicar o Teorema de separação de Eidelheit para os conjuntos convexos $\left(x_{0}+K\left(f_{1}, t\right)\right)$ e $C$, que garante a existência de $a \in \mathbb{R}$ e $f_{2} \in S_{X^{*}}$ satisfazendo a:

(i) $\operatorname{Re} f_{2}(x) \leq a$ para todo $x \in C$;

(ii) $\operatorname{Re} f_{2}(x) \geq a$ para todo $x \in\left(x_{0}+K\left(f_{1}, t\right)\right)$;

(iii) $\operatorname{Re} f_{2}(x)>a$ para todo $x \in\left(x_{0}+K\left(f_{1}, t\right)\right)^{\circ}$.

Além disso, analogamente ao que foi feito para $f_{1}$, podemos concluir que

$$
\inf \left\{\operatorname{Re} f_{2}(x): x \in B\right\}=\inf \left\{\operatorname{Re} f_{2}(x): x \in A\right\}-\delta
$$


Como $x_{0} \in C \cap\left(x_{0}+K\left(f_{1}, t\right)\right)$,

$$
\begin{aligned}
\sup \left\{\operatorname{Re} f_{2}(x): x \in C\right\} & =\operatorname{Re} f_{2}\left(x_{0}\right) \\
& =\inf \left\{\operatorname{Re} f_{2}(x): x \in\left(x_{0}+K\left(f_{1}, t\right)\right)\right\} \\
& \leq \inf \left\{\operatorname{Re} f_{2}(x): x \in B\right\} \\
& \stackrel{(2.2 .4)}{=} \inf \left\{\operatorname{Re} f_{2}(x): x \in A\right\}-\delta \\
& <\inf \left\{\operatorname{Re} f_{2}(x): x \in A\right\} .
\end{aligned}
$$

Já temos aqui que $\operatorname{Re} f_{2}\left(x_{0}\right)=\sup \left\{\operatorname{Re} f_{2}(x): x \in C\right\}<\inf \left\{\operatorname{Re} f_{2}(x): x \in A\right\}$. Resta provar que $\left\|f_{1}-f_{2}\right\| \leq \epsilon$.

Para isso, considere o espaço $X_{\mathbb{R}}$ (vide Observação 2.1.8). Pela Proposição 2.1.3, temos que $\operatorname{Re} f_{1}, \operatorname{Re} f_{2} \in S_{X_{\mathbb{R}}^{*}}$. Além disso, é claro que $K\left(f_{1}, t\right)=K\left(\operatorname{Re} f_{1}, t\right)\left(K\left(\operatorname{Re} f_{1}, t\right)\right.$ aqui é um cone no espaço $\left.X_{\mathbb{R}}\right)$, e assim $\operatorname{Re} f\left(x_{0}\right)=\inf \left\{\operatorname{Re} f_{2}(x): x \in\left(x_{0}+K\left(\operatorname{Re} f_{1}, t\right)\right)\right\}=\operatorname{Re} f\left(x_{0}\right)+\inf \left\{\operatorname{Re} f_{2}(x):\right.$ $\left.x \in K\left(\operatorname{Re} f_{1}, t\right)\right\}$. Portanto, $\operatorname{Re} f_{2}(x) \geq 0$ para todo $x \in K\left(\operatorname{Re} f_{1}, t\right)$. Lembremos também que $0<\epsilon<1$ e $t>(1+2 / \epsilon)$, por construção. Dessa forma, podemos aplicar o Lema 2.2.14 para o espaço $X_{\mathbb{R}}$ e os funcionais $\operatorname{Re} f_{1}, \operatorname{Re} f_{2}$ para concluir que $\left\|\operatorname{Re} f_{1}-\operatorname{Re} f_{2}\right\|_{X_{\mathbb{R}}^{*}} \leq \epsilon$. Assim, $\left\|f_{1}-f_{2}\right\|=$ $\left\|\operatorname{Re} f_{1}-\operatorname{Re} f_{2}\right\|_{X_{\mathbb{R}}^{*}} \leq \epsilon$, como queríamos demonstrar.

Finalmente, podemos apresentar os três Teoremas principais desta seção, demonstrados em [BP63] por Bishop e Phelps.

Resultados similares ao Teorema abaixo podem ser encontrados no livro Convex Functions, Monotone Operators and Differentiability, de Phelps, que trata-se de uma boa referência para mais detalhes sobre os conceitos tratados neste capítulo [Phe93].

Teorema 2.2.16 (Bishop-Phelps para funcionais suporte). Sejam $X \neq\{0\}$ espaço de Banach $e \emptyset \neq C \subset X$ subconjunto fechado, convexo e limitado. Então o conjunto dos funcionais suporte de $C$ é denso em $X^{*}$.

Demonstração. Afirmamos primeiro que o conjunto dos funcionais suporte de norma 1 de $C$ é denso em $S_{X^{*}}$. De fato, considere $f \in S_{X^{*}}$. Como $C$ é limitado, $\sup \{\operatorname{Re} f(x): x \in C\}$ é finito. Seja $y \in X$ tal que $\sup \{\operatorname{Re} f(x): x \in C\}<\operatorname{Re} f(y)$, e definamos $A \doteq\{y\}$. Temos que $\emptyset \neq C$ é fechado e convexo e $A$ é limitado. Pelo Teorema 2.2.15, dado $\epsilon>0$ qualquer, existe $g \in S_{X^{*}}$ funcional suporte de $C$ satisfazendo a $\|g-f\| \leq \epsilon$, o que mostra a afirmação.

Mostraremos agora o caso geral. Seja $f \neq 0 \in X^{*}$. Pelo que já vimos, dado $\epsilon>0$ qualquer, existe $g \in S_{X^{*}}$ que suporta $C$ em algum $x_{0} \in C$ satisfazendo a

$$
\left\|\frac{f}{\|f\|}-g\right\|<\frac{\epsilon}{\|f\|} .
$$

Logo, $\|f-\| f\|g\|<\epsilon \mathrm{e}$

$$
\begin{aligned}
\operatorname{Re}\|f\| g\left(x_{0}\right) & =\|f\| \operatorname{Re} g\left(x_{0}\right) \\
& =\|f\| \sup \{\operatorname{Re} g(x): x \in C\} \\
& =\sup \{\operatorname{Re}\|f\| g(x): x \in C\},
\end{aligned}
$$


ou seja, $\|f\| g \in X^{*}$ suporta $C$ em $x_{0}$. Resta mostrar que há elementos de $X^{*}$ que suportam $C$ de norma arbitrariamente pequena. Como $X \neq\{0\}$, dado $\epsilon>0$ existe $g \in B_{X^{*}}(0 ; \epsilon / 2) \backslash\{0\}$. Como já provamos, nesse caso existe $h \in X^{*}$ funcional suporte de $C$ tal que $\|g-h\|<\epsilon / 2$. Logo, $\|h\| \leq\|g-h\|+\|g\|<\epsilon$, o que encerra a prova do Teorema.

A hipótese da completude no espaço $X$ é essencial para o Teorema de Bishop-Phelps para funcionais suporte. De fato, Bishop e Phelps mostraram também em [BP63] que vale:

Teorema 2.2.17. Seja $X$ um espaço normado incompleto. Então existe um subconjunto $C \subset X$ convexo, limitado, fechado e de interior não-vazio tal que o conjunto dos funcionais suporte de $C$

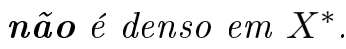

Demonstração. Suponhamos inicialmente que $X$ é espaço normado sobre $\mathbb{R}$.

Seja $\tilde{X}$ o completamento de $X$ (vide Proposição 1.1.7). Identificaremos $X$ com o subespaço denso de $\tilde{X}$ isométrico a $X$. Como $X$ é incompleto, existe $x \in \tilde{X} \backslash X$ com $\|x\|=1$. Pelo Corolário 1.1.16, existe $f \in S_{\tilde{X}^{*}}$ tal que $f(x)=\|x\|=1$. Definamos $D \doteq\{y \in \tilde{X}:\|y\| \leq 1$ e $f(y)=0\}=$ $B_{\tilde{X}} \cap \operatorname{Ker} f$. É claro que $D$ é convexo e fechado (pois $B_{\tilde{X}}$ e Ker $f$ o são). Seja também

$$
C^{\prime} \doteq\{z \in \tilde{X}: z=\lambda y+(1-\lambda) x, \operatorname{com} y \in D \text { e } \lambda \in[0,1]\}
$$

Como $D$ é limitado, $C^{\prime}$ é limitado. Provaremos a seguir que $C^{\prime}$ é convexo, fechado e $C^{\prime o} \neq \emptyset$.

Para começar, mostraremos que $C^{\prime}$ é convexo. Considere $z_{1}, z_{2} \in C^{\prime}$. Então existem $y_{1}, y_{2} \in D$ e $\lambda_{1}, \lambda_{2} \in[0,1]$ tais que

$$
\begin{aligned}
& z_{1}=\lambda_{1} y_{1}+\left(1-\lambda_{1}\right) x \\
& z_{2}=\lambda_{2} y_{2}+\left(1-\lambda_{2}\right) x
\end{aligned}
$$

Dado $\rho \in[0,1]$, precisamos mostrar que $z \doteq \rho z_{1}+(1-\rho) z_{2} \in C^{\prime}$. Temos que $\rho z_{1}+(1-\rho) z_{2}=$ $\rho \lambda_{1} y_{1}+(1-\rho) \lambda_{2} y_{2}+\left(\rho\left(1-\lambda_{1}\right)+(1-\rho)\left(1-\lambda_{2}\right)\right) x$, então se $M \doteq \rho\left(1-\lambda_{1}\right)+(1-\rho)\left(1-\lambda_{2}\right) \in[0,1]$, vale que

$$
z=(1-M)\left(\frac{\rho \lambda_{1} y_{1}+(1-\rho) \lambda_{2} y_{2}}{(1-M)}\right)+M x .
$$

Como $(1-M)=\rho \lambda_{1}+(1-\rho) \lambda_{2}$ e $D$ é convexo, por (2.2.5) concluímos que $z \in C^{\prime}$, como queríamos.

Afirmamos agora que $C^{\prime}$ é fechado. De fato, se $\left(z_{n}\right)_{n \in \mathbb{N}}$ é sequência de elementos em $C^{\prime}$ convergindo a algum $z \in \tilde{X}$, mostraremos que $z \in C^{\prime}$. Dado $n \in \mathbb{N}$, existem $y_{n} \in D$ e $\lambda_{n} \in[0,1]$ tais que $z_{n}=\lambda_{n} y_{n}+\left(1-\lambda_{n}\right) x$. Como $[0,1]$ é compacto, existe uma subsequência $\left(\lambda_{n_{k}}\right)_{k \in \mathbb{N}}$ de $\left(\lambda_{n}\right)_{n \in \mathbb{N}}$ tal que $\lambda_{n_{k}} \stackrel{\mathrm{k} \rightarrow \infty}{\longrightarrow} \lambda$, para algum $\lambda \in[0,1]$. Como $D$ é limitado, se $\lambda=0$ segue que $\lambda_{n_{k}} y_{n_{k}} \stackrel{\mathrm{k} \rightarrow \infty}{\longrightarrow} 0$. Nesse caso, $z_{n_{k}} \stackrel{\mathrm{k} \rightarrow \infty}{\longrightarrow} x$, e assim $z=x \in C^{\prime}$. Se $\lambda \neq 0$, existe $k_{0} \in \mathbb{N}$ tal que $\lambda_{n_{k}} \neq 0$ sempre que $k \geq k_{0}$. Logo, para $k \geq k_{0}$ podemos escrever

$$
y_{n_{k}}=\left(\frac{z_{n_{k}}-\left(1-\lambda_{n_{k}}\right) x}{\lambda_{n_{k}}}\right) \stackrel{\mathrm{k} \rightarrow \infty}{\longrightarrow}\left(\frac{z-(1-\lambda) x}{\lambda}\right) .
$$

Como $y_{n_{k}} \in D$ para todo $k \in \mathbb{N}$ e $D$ é fechado, $\left(\frac{z-(1-\lambda) x}{\lambda}\right) \in D$, o que implica que $z \in C^{\prime}$. 
Para finalizar, mostraremos que $B(x / 2 ; 1 / 8) \subset C^{\prime}$, donde seguirá que $C^{\prime o}$ é não-vazio. Tome $z \in B(x / 2 ; 1 / 8)$. Então $|f(z-x / 2)| \leq \| z-x / 2||<1 / 8$ e, como $f(x)=1$,

$$
\frac{3}{8}<f(z)<\frac{5}{8}
$$

Definamos agora

$$
y \doteq \frac{z}{(1-f(z))}-\frac{f(z) x}{(1-f(z))}
$$

Se $y$ pertencer a $D$, teremos que $z=f(z) x+(1-f(z)) y \in C^{\prime}$, portanto $B(x / 2 ; 1 / 8) \subset C^{\prime}$, e o problema acaba. Vamos provar isso. Notemos que (2.2.6) implica que

$$
\frac{1}{8(1-f(z))}<\frac{1}{3}
$$

Assim,

$$
\|y\| \leq\left\|\frac{(z-x / 2)}{(1-f(z))}\right\|+\left\|\frac{(x / 2-f(z) x)}{(1-f(z))}\right\|<\frac{1}{8(1-f(z))}+\frac{|1 / 2-f(z)|}{(1-f(z))}
$$

e por (2.2.7),

$$
\|y\|<\frac{1}{3}+\frac{8|1 / 2-f(z)|}{3}
$$

Usando que

$$
\left|\frac{1}{2}-f(z)\right|<\frac{1}{8}
$$

de (2.2.8) concluímos que $y \in B_{\tilde{X}}$. Além disso,

$$
f(y)=f\left(\frac{z}{(1-f(z))}-\frac{f(z) x}{(1-f(z))}\right)=0,
$$

donde vem que $y \in D$, como queríamos.

Definamos agora $C \doteq C^{\prime} \cap X$. Pelo que mostramos acima para $C^{\prime}$, temos que $C$ é convexo, limitado e fechado em $X$. Como $X$ é denso em $\tilde{X}$ e $C^{\prime \circ} \neq \emptyset$, pelo Corolário 1.1.11 temos ainda que $C$ é denso em $C^{\prime}$. Afirmamos que $C^{\mathrm{o}, X} \neq \emptyset$, em que $C^{\mathrm{o}, X}$ denota o interior de $C$ relativo a $X$. De fato, uma vez que $C^{\prime o} \neq \emptyset$, existe um aberto não-vazio $U$ de $\tilde{X}$ tal que $U \subset C^{\prime}$. Como $X$ é denso em $\tilde{X}, U \cap X$ é um aberto não-vazio de $X$ e vale que $U \cap X \subset C$, o que mostra a afirmação.

Lembremos que, conforme a Proposição 1.1.12, $P: \tilde{X}^{*} \rightarrow X^{*}$ dada por $P(g)=\left.g\right|_{X}$, para todo $g \in \tilde{X}^{*}$, é uma isometria entre $\tilde{X}^{*}$ e $X^{*}$. Vamos mostrar em seguida que dado $g \in \tilde{X}^{*}$, vale que $\sup \{P(g)(z): z \in C\}=\sup \left\{g(z): z \in C^{\prime}\right\}$ (os supremos são finitos, pois $C$ e $C^{\prime}$ são limitados). Se $g=0$, isso é claro. Suponhamos que $g \neq 0$. Como $C \subset C^{\prime}$ e $P(g)=\left.g\right|_{X}$,

$$
\sup \{P(g)(z): z \in C\} \leq \sup \left\{g(z): z \in C^{\prime}\right\}
$$

Para mostrar a inclusão oposta, tome $\epsilon>0$ e seja $z_{0} \in C^{\prime}$ tal que $\sup \left\{g(z): z \in C^{\prime}\right\}-\frac{\epsilon}{2}<g\left(z_{0}\right)$. 
Como $C$ é denso em $C^{\prime}$, existe $z_{1} \in C$ satisfazendo a $\left\|z_{0}-z_{1}\right\|<\frac{\epsilon}{2\|g\|}$. Logo,

$$
g\left(z_{0}-z_{1}\right) \leq\|g\| \frac{\epsilon}{2\|g\|}=\frac{\epsilon}{2}
$$

e assim,

$$
\sup \left\{g(z): z \in C^{\prime}\right\}-\epsilon<g\left(z_{0}\right)-\frac{\epsilon}{2}<g\left(z_{1}\right)=P(g)\left(z_{1}\right),
$$

o que prova que $\sup \{P(g)(z): z \in C\}=\sup \left\{g(z): z \in C^{\prime}\right\}$, como queríamos. Portanto, se $P(g) \in X^{*}$ suporta $C$ em $z_{0} \in C$, então $g \in \tilde{X}^{*}$ suporta $C^{\prime}$ no mesmo ponto.

Para finalizar, mostraremos que se $g \in \tilde{X}^{*}$ suporta $C^{\prime}$, então $\|g-f\| \geq \frac{1}{2}$. Seja $z \in C^{\prime}$ tal que $g(z)=\sup \left\{g(w): w \in C^{\prime}\right\}$. Por definição de $C^{\prime}$, existem $\lambda \in[0,1]$ e $y \in D$ tais que $z=\lambda y+(1-\lambda) x$. Temos que

$$
g(x) \leq g(z)=\lambda g(y-x)+g(x)
$$

donde vem que $g(y-x) \geq 0$, e assim

$$
(g-f)(y-x)=g(y-x)+1 \geq 1 .
$$

Então

$$
1 \leq|(g-f)(y-x)| \leq\|g-f|\||y-x\|\leq 2|| g-f\|,
$$

ou seja, $\|g-f\| \geq \frac{1}{2}$. Usando os resultados mostrados acima e novamente que $P$ é isometria, concluímos que dado $h \in X^{*}$ que suporta $C$, digamos $h=P(g), g \in \tilde{X}^{*}$, vale que $g$ suporta $C^{\prime}$ e então $\|P(g)-P(f)\|_{X^{*}}=\|g-f\| \geq \frac{1}{2}$. Portanto, o conjunto dos funcionais que suportam $C$ não é denso em $X^{*}$, com $C$ de interior não-vazio, limitado, convexo e fechado.

Agora suponhamos que $X$ é espaço normado sobre $\mathbb{C}$, e consideremos o espaço normado $X_{\mathbb{R}}$ (vide Observação 2.1.8). Pelo que foi mostrado, existe $C \subset X_{\mathbb{R}}$ fechado, convexo, limitado e de interior não-vazio tal que o conjunto dos funcionais em $X_{\mathbb{R}}^{*}$ que suportam $C$ não é denso em $X_{\mathbb{R}}^{*}$. Como $X$ e $X_{\mathbb{R}}$ têm a mesma topologia, o conjunto $C$ também é fechado e tem interior não-vazio em relação a $X$. Usando que $R: X^{*} \ni f \mapsto \operatorname{Re} f \in X_{\mathbb{R}}^{*}$ é bijeção $\mathbb{R}$-linear que preserva normas e que $f \in X^{*}$ suporta um subconjunto de $X$ se, e somente se, Re $f \in X_{\mathbb{R}}^{*}$ também o suporta, concluímos que o conjunto dos funcionais em $X^{*}$ que suportam $C$ também não é denso em $X^{*}$, encerrando a demonstração do teorema.

O Corolário seguinte é um resultado curioso que decorre dos dois teoremas anteriores.

Corolário 2.2.18. Um espaço normado $X \neq\{0\}$ é um espaço de Banach se, e somente se, para todo $C \subset X$ não-vazio, fechado, convexo e limitado, o conjunto dos funcionais que suportam $C$ é denso em $X^{*}$.

A seguir apresentamos o Teorema de Bishop-Phelps, principal objetivo desta seção.

Teorema 2.2.19 (Bishop-Phelps). Todo espaço de Banach é subreflexivo. 
Demonstração. O espaço $X=\{0\}$ é subreflexivo, pois nesse caso $X^{*}=\{0\}$ e o funcional nulo trivialmente atinge sua norma. Suponhamos então que $X \neq\{0\}$. O conjunto $B_{X}$ é fechado, convexo e limitado. Pelo Teorema de Bishop-Phelps para funcionais suporte (2.2.16), o conjunto dos funcionais suporte de $B_{X}$ é denso em $X^{*}$. O Corolário 2.1.4 garante então que $\mathcal{N} \mathcal{A}_{X}$ é denso em $X^{*}$, isto é, $X$ é subreflexivo.

\subsection{Teorema de Bishop-Phelps-Bollobás}

Nesta seção, apresentamos a demonstração do Teorema de Bishop-Phelps-Bollobás [Bol70], uma versão quantitativa e mais forte do Teorema de Bishop-Phelps.

Começaremos com um Lema. A ideia da sua demonstração é similar à do Teorema 2.2.15.

Lema 2.3.1. Sejam $X$ um espaço de Banach sobre $\mathbb{R}, f_{1} \in S_{X^{*}}, 0<\epsilon<1$ e $z \in B_{X}$ tal que $f_{1}(z) \neq 0$. Então existem $x_{0} \in B_{X}$ e $f_{2} \in S_{X^{*}}$ tais que

$$
\left\|f_{2}-f_{1}\right\|<\epsilon,\left\|x_{0}-z\right\| \leq\left(\frac{2}{\epsilon}+1\right) \frac{f_{1}\left(x_{0}-z\right)}{\left|f_{1}(z)\right|}
$$

e $f_{2}$ suporta $B_{X}$ em $x_{0}$.

Demonstração. Notemos inicialmente que se $\left|f_{1}(z)\right|=1$, então $f_{1}$ suporta $B_{X}$ em $+z$ ou $-z$. Definindo nesse caso $x_{0}$ como o ponto suporte e $f_{2} \doteq f_{1}$, a conclusão do Lema segue. De fato, se o ponto suporte for $z, f_{1}(z)=1$ e teremos $\left\|f_{2}-f_{1}\right\|=\left\|x_{0}-z\right\|=0 \mathrm{e}$

$$
\left(\frac{2}{\epsilon}+1\right) \frac{f_{1}\left(x_{0}-z\right)}{\left|f_{1}(z)\right|}=0
$$

Se for $-z$, teremos $f_{1}(-z)=1$ e assim

$$
\left\|x_{0}-z\right\| \leq 2<\left(\frac{2}{\epsilon}+1\right) 2=\left(\begin{array}{l}
2 \\
\bar{\epsilon}
\end{array}+1\right) \frac{f_{1}\left(x_{0}-z\right)}{\left|f_{1}(z)\right|} .
$$

Suponhamos então que $\left|f_{1}(z)\right|<1$. Seja

$$
t \doteq\left(1+\frac{2}{\epsilon}\right) \frac{1}{\left|f_{1}(z)\right|}
$$

É claro que $t>\left(1+\frac{2}{\epsilon}\right)>1$. Como $X$ é espaço de Banach, $B_{X}$ é completo. Além disso, $\left\{f_{1}(x)\right.$ : $\left.x \in B_{X}\right\}$ é limitado, já que $B_{X}$ o é. Pelo Lema 2.2.11, existe $x_{0} \in B_{X}$ tal que $x_{0} \in\left(z+K\left(f_{1}, t\right)\right)$ e $\left(x_{0}+K\left(f_{1}, t\right)\right)$ suporta $B_{X}$ em $x_{0}$. Ou seja, $\left\|x_{0}-z\right\| \leq t f_{1}\left(x_{0}-z\right)$ e $\left(x_{0}+K\left(f_{1}, t\right)\right) \cap B_{X}=$ $\left\{x_{0}\right\}$. Como já discutido na demonstração do Teorema 2.2.15, a Observação 2.2.10 implica que $\left(x_{0}+K\left(f_{1}, t\right)\right)^{\circ} \cap B_{X}=\emptyset$, e pela Proposição $2.2 .9, K\left(f_{1}, t\right)^{\circ} \neq \emptyset$. Logo, podemos aplicar o Teorema de separação de Eidelheit para os conjuntos convexos $\left(x_{0}+K\left(f_{1}, t\right)\right)$ e $B_{X}$, que garante a existência de $a \in \mathbb{R}$ e $f_{2} \in S_{X^{*}}$ satisfazendo a:

(i) $f_{2}(x) \leq a$ para todo $x \in B_{X}$; 
(ii) $f_{2}(x) \geq a$ para todo $x \in\left(x_{0}+K\left(f_{1}, t\right)\right)$;

(iii) $f_{2}(x)>a$ para todo $x \in\left(x_{0}+K\left(f_{1}, t\right)\right)^{\circ}$.

Como $x_{0} \in B_{X} \cap\left(x_{0}+K\left(f_{1}, t\right)\right)$,

$$
\begin{aligned}
f_{2}\left(x_{0}\right) & =\sup \left\{f_{2}(x): x \in B_{X}\right\} \\
& =\inf \left\{f_{2}(x): x \in\left(x_{0}+K\left(f_{1}, t\right)\right)\right\} \\
& =f_{2}\left(x_{0}\right)+\inf \left\{f_{2}(x): x \in K\left(f_{1}, t\right)\right\} .
\end{aligned}
$$

Assim, $f_{2}$ suporta $B_{X}$ em $x_{0}$ e $f_{2}(x) \geq 0$ para todo $x \in K\left(f_{1}, t\right)$. Tomemos $\epsilon^{\prime} \in(0, \epsilon)$ satisfazendo a $t>\left(1+\frac{2}{\epsilon^{\prime}}\right)>\left(1+\frac{2}{\epsilon}\right)$. Pelo Lema 2.2.14, $\left\|f_{2}-f_{1}\right\| \leq \epsilon^{\prime}<\epsilon$. Como também temos que

$$
\left\|x_{0}-z\right\| \leq t f_{1}\left(x_{0}-z\right)=\left(1+\frac{2}{\epsilon}\right) \frac{f_{1}\left(x_{0}-z\right)}{\left|f_{1}(z)\right|}
$$

segue a conclusão do Lema.

Agora podemos apresentar a demonstração do Teorema de Bishop-Phelps-Bollobás.

Teorema 2.3.2 (Bishop-Phelps-Bollobás). Sejam X um espaço de Banach e $0<\epsilon<1$. Dados $x \in B_{X}$ e $f \in S_{X^{*}}$ tais que $|1-f(x)|<\frac{\epsilon^{2}}{4}$, existem $y \in S_{X}$ e $g \in S_{X^{*}}$ satisfazendo a

$$
g(y)=1,\|y-x\|<\epsilon e\|g-f\|<\epsilon .
$$

Demonstração. Suponhamos inicialmente que $X$ é espaço de Banach sobre $\mathbb{R}$.

Pelo Lema 2.3.1, existem $g \in S_{X^{*}}$ e $y \in B_{X}$ tais que

$$
\|g-f\|<\epsilon,\|y-x\|<\left(1+\frac{2}{\epsilon}\right) \frac{f(y-x)}{|f(x)|} \text { e } g \text { suporta } B_{X} \text { em } y .
$$

Notemos aqui que como $y$ é ponto suporte de $B_{X}$, pela Proposição 2.1.6, $y \in S_{X}$. Vale ainda que $g(y)=\sup \left\{g(z): z \in B_{X}\right\}=\|g\|=1$, pela Proposição 2.1.3. Como $0 \leq f(y-x) \leq 1-f(x)$, temos

$$
\|y-x\|<\left(1+\frac{2}{\epsilon}\right) \frac{1-f(x)}{|f(x)|}<\left(1+\frac{2}{\epsilon}\right) \frac{\epsilon^{2}}{4|f(x)|}
$$

Usando que $1-|f(x)| \leq|f(x)-1|<\frac{\epsilon^{2}}{4}$, obtemos $|f(x)|>1-\frac{\epsilon^{2}}{4}$, e por (2.3.1),

$$
\begin{aligned}
\|y-x\| & <\left(1+\frac{2}{\epsilon}\right) \frac{\epsilon^{2}}{4\left(1-\frac{\epsilon^{2}}{4}\right)} \\
& =\frac{\epsilon}{2\left(1-\frac{\epsilon^{2}}{4}\right)}+\frac{\epsilon^{2}}{4\left(1-\frac{\epsilon^{2}}{4}\right)} .
\end{aligned}
$$


Como $0<\epsilon<1$, vem que $1-\frac{\epsilon^{2}}{4}>\frac{3}{4}$, e então

$$
\|y-x\|<\frac{4 \epsilon}{2 \cdot 3}+\frac{4 \epsilon^{2}}{4 \cdot 3} \leq \epsilon
$$

encerrando a demonstração deste caso.

Suponhamos agora que $X$ é espaço normado sobre $\mathbb{C}$, e consideremos o espaço $X_{\mathbb{R}}$ e a aplicação $R: X^{*} \ni f \mapsto \operatorname{Re} f \in X_{\mathbb{R}}^{*}$. Vimos na Observação 2.1.8 que $R$ é bijeção que preserva normas. Temos que

$$
|1-\operatorname{Re} f(x)|=\operatorname{Re}(1-f(x)) \leq|1-f(x)|<\frac{\epsilon^{2}}{4} .
$$

Aplicando então o caso demonstrado acima para $X_{\mathbb{R}}, x \in B_{X_{\mathbb{R}}}$ e $\operatorname{Re} f \in S_{X_{\mathbb{R}}^{*}}$, garantimos a existência de $g \in S_{X^{*}}$ e $y \in S_{X}$ satisfazendo a $\operatorname{Re} g(y)=1,\|y-x\| \leq \epsilon$ e $\|\operatorname{Re}(g)-\operatorname{Re}(f)\|_{X_{\mathbb{R}}^{*}}<\epsilon$. Portanto, também vale que $g(y)=1$ e $\|g-f\|<\epsilon$, como queríamos.

Notemos que se a hipótese $|1-f(x)|<\frac{\epsilon^{2}}{4}$ do Teorema de Bishop-Phelps-Bollobás for enfraquecida para $|f(x)|>1-\frac{\epsilon^{2}}{4}$, podemos concluir que existem elementos $g \in S_{X^{*}}$ e $y \in S_{X}$ satisfazendo a $\|g-f||<\epsilon\| x-,y \|<\epsilon$ e $|g(y)|=1$ (e não mais a $g(y)=1$ ). De fato, seja $\lambda \in S_{\mathbb{K}}$ tal que $f(\lambda x)=|f(x)|$ e considereremos $x^{\prime} \doteq \lambda x$. Então $x^{\prime} \in B_{X}$ e

$$
\left|1-f\left(x^{\prime}\right)\right|=1-f\left(x^{\prime}\right)=1-|f(x)|<\frac{\epsilon^{2}}{4} .
$$

Pelo Teorema de Bishop-Phelps-Bollobás, existem $g \in S_{X^{*}}$ e $y^{\prime} \in S_{X}$ tais que $g\left(y^{\prime}\right)=1,\left\|y^{\prime}-x^{\prime}\right\|<\epsilon$ e $\|g-f\|<\epsilon$. Então definindo $y \doteq \lambda^{-1} y^{\prime} \in S_{X}$, teremos $\left\|y^{\prime}-x^{\prime}\right\|=\|y-x\|<\epsilon,\|f-g\|<\epsilon$ e $|g(y)|=1$. 


\section{Capítulo 3}

\section{Operadores que atingem a norma}

É natural questionar se versões análogas aos Teoremas de Bishop-Phelps e Bishop-PhelpsBollobás valem quando tratamos de operadores lineares e contínuos definidos entre dois espaços de Banach $X$ e $Y$. Da mesma forma que fizemos para os funcionais, dizemos que um operador $T \in \mathcal{L}(X, Y)$ atinge sua norma se existe $x_{0} \in B_{X}$ tal que $\left\|T\left(x_{0}\right)\right\|=\|T\|$. Definimos ainda o conjunto

$$
\mathcal{N} \mathcal{A}(X, Y) \doteq\{T \in \mathcal{L}(X, Y): T \text { atinge sua norma }\}
$$

Antes mesmo de Bollobás apresentar o Teorema de Bishop-Phelps-Bollobás, Lindenstrauss já havia obtido muitos resultados notáveis no sentido de obter generalizações do Teorema de BishopPhelps a operadores no seu artigo [Lin63], de 1963. Estudaremos neste capítulo alguns dos resultados de [Lin63]. Entretanto, nosso principal objetivo é tratar da extensão do Teorema de Bishop-PhelpsBollobás a operadores, através da Propriedade de Bishop-Phelps-Bollobás para operadores (BPBp), introduzida por Acosta et al. no artigo [AAGM08], de 2008.

Na primeira seção, estudaremos os conceitos básicos que serão usados no restante do capítulo, como a propriedade $\beta$ de Lindenstrauss, pontos exposto e fortemente exposto em um espaço normado e famílias fortemente e uniformemente expostas por uma função. Na seção seguinte, será discutida brevemente a extensão do Teorema de Bishop-Phelps a operadores, com alguns resultados de [Lin63]. Na última seção, estudaremos a Propriedade de Bishop-Phelps-Bollobás para operadores em certos pares de espaços de Banach, baseando-nos principalmente em [AAGM08] e [AGKM17].

\subsection{Conceitos iniciais}

Os conceitos de ponto exposto e fortemente exposto definidos abaixo foram amplamente usados por Lindenstrauss ao estudar operadores que atingem a norma.

Definição 3.1.1. Seja $C$ um subconjunto convexo de um espaço de Banach $X$. Um ponto $x \in C$ é dito ponto exposto de $C$ se existe $f \in X^{*}$ satisfazendo a $\operatorname{Re} f(y)<\operatorname{Re} f(x)$ para todo $y \in C$ tal que $y \neq x$.

Observação 3.1.2. Nas condições da definição acima, no caso $X \neq\{0\}$, todo ponto exposto de $C$ é ponto suporte de $C$. De fato, caso $C$ tenha apenas um elemento, este claramente será ponto exposto e ponto suporte em relação a qualquer funcional não-nulo em $X^{*}$. Caso $C$ tenha mais de um elemento, o funcional $f$ satisfazendo a definição para $x$ será não-nulo e obviamente $f$ suporta $C$ em $x$. Na verdade, $x$ é o único ponto de $C$ que satisfaz a essa última afirmação. 
Definição 3.1.3. Seja $C$ um subconjunto convexo de um espaço de Banach $X$. Um ponto $x \in C$ é dito ponto fortemente exposto de $C$ se existe $f \in X^{*}$ satisfazendo a:

(i) $\operatorname{Re} f(y) \leq \operatorname{Re} f(x)$ para todo $y \in C$.

(ii) Se $\left(x_{n}\right)_{n \in \mathbb{N}}$ é sequência de elementos em $C$ tal que $\operatorname{Re} f\left(x_{n}\right) \rightarrow \operatorname{Re} f(x)$, então $x_{n} \rightarrow x$.

Observe que o item (i) da definição acima poderia ser substituído pela afirmação:

(i)' $\operatorname{Re} f(y)<\operatorname{Re} f(x)$ para todo $y \in C$ tal que $y \neq x$.

De fato, dado $y \in C$ tal que $\operatorname{Re} f(x)=\operatorname{Re} f(y)$, tomando a sequência constante e igual a $y$ em $C$, pelo item (ii) da Definição acima obtemos $y=x$. Assim, por (i), $\operatorname{Re} f(y)<\operatorname{Re} f(x)$ se $y \in C \backslash\{x\}$. Em particular, todo ponto fortemente exposto de $C$ é ponto exposto de $C$ em relação ao mesmo funcional $f \in X^{*}$.

Observação 3.1.4. Se $x_{0} \in C$ é ponto exposto (ou fortemente exposto) de $C$ pelo funcional $f \in$ $X^{*} \backslash\{0\}$, então também é ponto exposto (ou fortemente exposto) pelo funcional $\frac{f}{\|f\|} \in S_{X^{*}}$.

Mostraremos através dos exemplos a seguir que nem todo ponto suporte é ponto exposto, e que nem todo ponto exposto é ponto fortemente exposto.

Exemplo 3.1.5. Consideremos o espaço de Banach $X \doteq \ell_{\infty}^{2}$ sobre $\mathbb{R}$ e $C \doteq B_{X}$. Afirmamos que $(1,0)$ é ponto suporte mas não é ponto exposto de $C$.

De fato, para $f: \mathbb{R}^{2} \rightarrow \mathbb{R}$ dada por $f(x, y)=x$ para todo $(x, y) \in X$, temos que $\|f\|=1=$ $f((1,0))$, donde concluímos que $(1,0)$ é ponto suporte de $C$. Suponhamos que $(1,0)$ seja ponto exposto de $C$ em relação a algum $g \in X^{*}$. Então $g(1, \pm 1)=g(1,0) \pm g(0,1)<g(1,0)$, e portanto $\pm g(0,1)<0$, absurdo.

Antes de iniciar o exemplo abaixo, lembremos que toda sequência ortonormal em um espaço de Hilbert converge a 0 na topologia fraca.

Exemplo 3.1.6. Consideremos o espaço de Banach $X \doteq \ell_{2}$ sobre $\mathbb{R}$ e $C \doteq\left\{x \in B_{\ell_{2}}: x_{i} \geq\right.$ 0 para todo $i \in \mathbb{N}\}$. Afirmamos que $0 \in \ell_{2}$ é ponto exposto de $C$ que não é ponto fortemente exposto.

Inicialmente, notemos que $C$ é convexo. Seja $f: \ell_{2} \rightarrow \mathbb{R}$ dada por

$$
f(x)=\sum_{i=1}^{\infty} \frac{-x_{i}}{2^{i}} \text { para todo } x \in \ell_{2} .
$$

Como a série acima converge absolutamente para cada $x \in \ell_{2}$, a função $f$ está bem definida, e é linear. Além disso, se $x \in B_{\ell_{2}}$ é claro que $\left|x_{i}\right| \leq 1$ para todo $i \in \mathbb{N}$. Logo, para todos $n \in \mathbb{N}$ e $x \in B_{\ell_{2}}$,

$$
\left|\sum_{i=1}^{n} \frac{-x_{i}}{2^{i}}\right| \leq \sum_{i=1}^{n} \frac{\left|x_{i}\right|}{2^{i}} \leq \sum_{i=1}^{n} \frac{1}{2^{i}} \leq 1 .
$$

Levando $n \rightarrow \infty$ na desigualdade acima, concluímos que $|f(x)| \leq 1$ para todo $x \in B_{\ell_{2}}$, e então $f \in \ell_{2}^{*}$. Se $x \in C \backslash\{0\}$, teremos que $f(x)<0$, e se $x=0 \in C, f(x)=0$. Então 0 é ponto exposto de $C$ pelo funcional $f \in \ell_{2}^{*}$. 
Mostraremos agora que 0 não é ponto fortemente exposto de $C$. Tendo em vista que $\left(e_{n}\right)_{n \in \mathbb{N}}$ é sequência em $C$ ortonormal no espaço de Hilbert $\ell_{2}$, vale que $e_{n} \stackrel{w}{\longrightarrow} 0$. Logo, para todo $g \in \ell_{2}^{*}$ temos $g\left(e_{n}\right) \rightarrow 0=g(0)$, mesmo que $e_{n} \nrightarrow 0$. Portanto, 0 não é ponto fortemente exposto de $C$.

O conceito seguinte também foi baseado no artigo [Lin63] de Lindenstrauss, mas trata-se da versão definida em [AGKM17].

Definição 3.1.7. Sejam $Y$ um espaço de Banach, $E \subset S_{Y}$ e $F: E \rightarrow S_{Y^{*}}$. Dizemos que a família $E$ é fortemente $e$ uniformemente exposta por $F$ se para todo $\epsilon>0$ existe $\delta>0$ satisfazendo a:

$$
\left(y \in B_{Y}, e \in E, \operatorname{Re} F(e)(y)>1-\delta\right) \Rightarrow\|y-e\|<\epsilon .
$$

A Proposição abaixo segue sem muita dificuldade.

Proposição 3.1.8. Sejam $Y$ um espaço de Banach e $E \subset S_{Y}$ uma família fortemente e uniformemente exposta por $F: E \rightarrow S_{Y^{*}}$. Então todo $e \in E$ é ponto fortemente exposto de $B_{Y}$, pelo funcional $F(e) \in S_{Y^{*}}$.

Demonstração. Pela Proposição 2.1.3,

$$
\sup \left\{\operatorname{Re} F(e)(y): y \in B_{Y}\right\}=\|F(e)\|=1 \text {, para todo } e \in E .
$$

Fixe $e \in E$. Por (3.1.1), existe $\left(y_{n}\right)_{n \in \mathbb{N}}$ sequência em $B_{Y}$ tal que $\operatorname{Re} F(e)\left(y_{n}\right) \rightarrow 1$, e por hipótese de família fortemente e uniformemente exposta, isso implica que $y_{n} \rightarrow e$. Logo, $\operatorname{Re} F(e)\left(y_{n}\right) \rightarrow$ $\operatorname{Re} F(e)(e)$, e a unicidade do limite em $\mathbb{K}$ nos permite concluir que $\operatorname{Re} F(e)(e)=1$. Assim, $\operatorname{Re} F(e)(y) \leq$ $\operatorname{Re} F(e)(e)$ para todo $y \in B_{Y}$.

Agora, se $\left(z_{n}\right)_{n \in \mathbb{N}}$ é sequência em $B_{Y}$ tal que $\operatorname{Re} F(e)\left(z_{n}\right) \rightarrow 1=\operatorname{Re} F(e)(e)$, novamente da hipótese concluímos que $z_{n} \rightarrow e$, encerrando a demonstração.

A seguir daremos um exemplo de uma família e função satisfazendo à Definição 3.1.7.

Exemplo 3.1.9. Seja $Y$ um espaço de Banach uniformemente convexo (vide seção 1.5). Pelo Corolário 1.1.16, para cada $e \in S_{Y}$ existe $f_{e} \in S_{Y^{*}}$ tal que $f_{e}(e)=\|e\|=1$. Definamos $F: S_{Y} \ni e \mapsto$ $f_{e} \in S_{Y^{*}}$. Mostraremos que a família $S_{Y}$ é fortemente e uniformemente exposta por $F$.

Como $Y$ é uniformemente convexo, dado $\epsilon>0$ existe $\delta=\delta(\epsilon)>0$ tal que

$$
\left(x, y \in B_{Y}, \frac{\|x+y\|}{2}>1-\delta\right) \Rightarrow\|x-y\|<\epsilon .
$$

Logo, se

$$
y \in B_{Y}, e \in S_{Y}, \operatorname{Re} F(e)(y)>1-\delta,
$$

teremos que $\operatorname{Re} f_{e}(y)>1-\delta$, então

$$
\operatorname{Re} f_{e}(y)=\operatorname{Re} f_{e}(e+y)-\operatorname{Re} f_{e}(e)=\operatorname{Re} f_{e}(e+y)-1>1-\delta,
$$

e finalmente

$$
\frac{\|e+y\|}{2} \geq \operatorname{Re} f_{e}\left(\frac{e+y}{2}\right)>1-\frac{\delta}{2}>1-\delta
$$


o que implica que $\|e-y\|<\epsilon$, como queríamos demonstrar.

A seguir passaremos a discutir a propriedade $\beta$ de Lindenstrauss, introduzida em [Lin63]. Espaços com esta propriedade satisfazem a muitos resultados interessantes a respeito de operadores que atingem da norma, alguns dos quais serão apresentados neste capítulo.

Definição 3.1.10. Dizemos que um espaço de Banach $Y$ satisfaz a propriedade $\beta$ (de Lindenstrauss) se existem conjuntos $\left\{y_{\alpha}: \alpha \in \Lambda\right\} \subset S_{Y},\left\{f_{\alpha}: \alpha \in \Lambda\right\} \subset S_{Y^{*}}$ e um real $\rho \in[0,1)$ tais que:

(i) $f_{\alpha}\left(y_{\alpha}\right)=1$, para todo $\alpha \in \Lambda$;

(ii) $\left|f_{\alpha}\left(y_{\gamma}\right)\right| \leq \rho$, para todos $\alpha, \gamma \in \Lambda$ tais que $\alpha \neq \gamma$;

(iii) $\|y\|=\sup \left\{\left|f_{\alpha}(y)\right|: \alpha \in \Lambda\right\}$, para todo $y \in Y$.

Exemplo 3.1.11. (a) Todo espaço normado unidimensional $X$ satisfaz a propriedade $\beta$.

De fato, consideremos $x \in S_{X}$ e $f_{x} \in S_{X^{*}}$ satisfazendo a $f_{x}(x)=1$. Os conjuntos $\{x\}$ e $\left\{f_{x}\right\}$ satisfazem a (i)-(iii) da Definição 3.1 .10 para $\rho=0$.

(b) Os espaços $\ell_{\infty}, c_{0}$ e $\ell_{\infty}^{n}$, para $n \in \mathbb{N}$, satisfazem a propriedade $\beta$.

Faremos a demonstração para $\ell_{\infty}$, e para os demais casos a conclusão seguirá por um argumento análogo. Seja $\Lambda \doteq \mathbb{N}$, e para cada $n \in \mathbb{N}$ consideremos $e_{n} \in S_{\ell_{\infty}}$ e $P_{n}: \ell_{\infty} \ni x \mapsto x_{n} \in \mathbb{K}$ em $S_{\ell_{\infty}^{*}}$. Os conjuntos $\left\{e_{n}: n \in \Lambda\right\}$ e $\left\{P_{n}: n \in \Lambda\right\}$ satisfazem a (i)-(iii) da Definição 3.1.10 para $\rho=0$.

(c) Para todo espaço topológico compacto Hausdorff $K$ contendo um subconjunto $D$ denso de pontos isolados, o espaço de Banach $C(K)$ satisfaz a propriedade $\beta$.

De fato, dado $x \in D$, seja $f_{x}$ a função característica do conjunto $\{x\}$. Vamos mostrar que $f_{x} \in C(K)$. Para isso, considere $y \in K$. Se $y \neq x$ e $V$ é uma vizinhança de $f_{x}(y)=0$ em $\mathbb{K}$, temos que $K \backslash\{x\}$ é aberto de $K$ contendo $y$ e $f_{x}(K \backslash\{x\})=\{0\} \subset V$. Mostramos por enquanto que $f_{x}$ é contínua em todo ponto de $K$ diferente de $x$. Como o ponto $x$ é isolado, $\{x\}$ é aberto de $K$ e dada uma vizinhança $V$ de $f_{x}(x)=1$, temos que $f_{x}(\{x\})=\{1\} \subset V$. Então $f_{x} \in C(K)$ para todo $x \in D$. Além disso, $\left\|f_{x}\right\|_{\infty}=1$.

Agora, para cada $x \in D$, definamos o funcional

$$
\begin{aligned}
F_{x}: C(K) & \rightarrow \mathbb{K} \\
f & \mapsto f(x) .
\end{aligned}
$$

Como $\left|F_{x}(f)\right|=|f(x)| \leq\|f\|_{\infty}$ para todo $f \in C(K)$ e $F_{x}\left(f_{x}\right)=1$, vale que $F_{x} \in S_{C(K)^{*}}$ para todo $x \in D$. Vamos mostrar que os conjuntos $\left\{f_{x}: x \in D\right\}$ e $\left\{F_{x}: x \in D\right\}$ satisfazem a (i)-(iii) da Definição 3.1 .10 para $\rho=0$.

Para verificar (i), basta notar que $F_{x}\left(f_{x}\right)=1$ para todo $x \in D$, e para (ii), que $F_{x}\left(f_{y}\right)=f_{y}(x)=$ 0 , para todos $x, y \in D$ tais que $x \neq y$. Vamos mostrar (iii). Como $K$ é compacto, dado $f \in C(K)$ existe $y_{0} \in K$ tal que $\left|f\left(y_{0}\right)\right|=\|f\|_{\infty}$. A função $|f|: K \ni x \mapsto|f(x)| \in \mathbb{K}$ também é contínua, então dado $\epsilon>0$ existe uma vizinhança $V_{0}$ de $y_{0}$ em $K$ tal que $|f|\left(V_{0}\right) \subset B\left(\left|f\left(y_{0}\right)\right| ; \epsilon\right)$. Como $D$ é denso, existe $x_{0} \in V_{0} \cap D$. Assim,

$$
|| F_{x_{0}}(f)|-|\left|f \left\|_{\infty}|=|\left|f \|_{\infty}-\right| f\left(x_{0}\right) \mid<\epsilon\right.\right.
$$


Mostramos que, dado $\epsilon>0$, podemos encontrar $x_{0} \in D$ tal que $\|f\|_{\infty}-\left|F_{x_{0}}(f)\right|<\epsilon$. Portanto, $\|f\|_{\infty}=\sup \left\{\left|F_{x}(f)\right|: x \in D\right\}$ para todo $f \in C(K)$.

\subsection{Bishop-Phelps para operadores}

O Teorema de Bishop-Phelps, mostrado na seção 2.2 do trabalho, afirma que $\mathcal{N} \mathcal{A}(X, \mathbb{K})$ é denso em $\mathcal{L}(X, \mathbb{K})$ para qualquer espaço de Banach $X$. Dados dois espaços de Banach $X$ e $Y$, poderíamos nos perguntar se vale que $\mathcal{N} \mathcal{A}(X, Y)$ é denso em $\mathcal{L}(X, Y)$. Ou ainda, que condições devem ser colocadas sobre $X$ (ou $Y$ ) para que $\mathcal{N} \mathcal{A}(X, Y)$ seja denso em $\mathcal{L}(X, Y)$ para qualquer espaço de Banach $Y$ (ou $X)$ ? Dado um espaço de Banach $X$ (ou $Y$ ), existe uma caracterização de espaços de Banach $Y$ (ou $X$ ) tais que $\mathcal{N} \mathcal{A}(X, Y)$ seja denso em $\mathcal{L}(X, Y)$ ? São perguntas desse tipo que uma extensão do Teorema de Bishop-Phelps a operadores deve responder.

Como já discutimos, o estudo de tais extensões teve início em 1963, quando Lindenstrauss publicou seu artigo [Lin63], que continha muitos resultados notáveis nesse sentido. Mostrou, por exemplo, que se o espaço de Banach $Y$ tem a propriedade $\beta$, então $\mathcal{N} \mathcal{A}(X, Y)$ é denso em $\mathcal{L}(X, Y)$ para todo espaço de Banach $X$, e que se $X$ é reflexivo, então $\mathcal{N} \mathcal{A}(X, Y)$ é denso em $\mathcal{L}(X, Y)$ para todo espaço de Banach $Y$.

Nesta seção, estudamos algumas propriedades simples da extensão do Teorema de Bishop-Phelps a operadores e apresentamos a demonstração do resultado de [Lin63] que afirma que se $Y$ for estritamente convexo e existir um operador não-compacto definido entre $c_{0}$ e $Y$, então $\mathcal{N} \mathcal{A}\left(c_{0}, Y\right)$ não é denso em $\mathcal{L}\left(c_{0}, Y\right)$. A partir deste resultado, mostramos um exemplo concreto de espaço $Y$ para o qual $\mathcal{N} \mathcal{A}\left(c_{0}, Y\right)$ não é denso em $\mathcal{L}\left(c_{0}, Y\right)$.

Para começar, observemos que como $\mathcal{N} \mathcal{A}(X, \mathbb{K})$ é denso em $\mathcal{L}(X, \mathbb{K})$ para qualquer espaço de Banach $X$ e espaços normados unidimensionais sempre são isométricos, segue a seguinte proposição.

Proposição 3.2.1. Se $X$ e $Y$ são espaços de Banach e $\operatorname{dim} Y=1$, então $\overline{\mathcal{N} \mathcal{A}(X, Y)}=\mathcal{L}(X, Y)$.

A seguir enunciamos outro resultado simples.

Proposição 3.2.2. Se $X$ e $Y$ são espaços de Banach e $\operatorname{dim} X<\infty$, então $\mathcal{N} \mathcal{A}(X, Y)=\mathcal{L}(X, Y)$.

Demonstração. Basta observar que nesse caso $B_{X}$ é compacta, então todo operador em $\mathcal{L}(X, Y)$ atinge sua norma.

A seguir faremos alguns resultados que nos permitirão construir um exemplo de espaço de Banach $Y$ para o qual $\mathcal{N} \mathcal{A}\left(c_{0}, Y\right)$ não é denso em $\mathcal{L}\left(c_{0}, Y\right)$. A existência deste exemplo será relevante na próxima seção.

A demonstração da Proposição abaixo é baseada na demonstração de um resultado feito por Lindenstrauss em [Lin63], mas trata-se da versão demonstrada em [AAGM08].

Proposição 3.2.3. Sejam $Y$ um espaço de Banach estritamente convexo e $T \in \mathcal{L}(X, Y)$, em que $X=c_{0}, \ell_{\infty}$ ou $\ell_{\infty}^{n}$, para $n \in \mathbb{N}$. Se $\|T(x)\|=\|T\|$ para $x \in S_{X}$, então $T\left(e_{k}\right)=0$ sempre que $k \in\left\{i \in \mathbb{N}:\left|x_{i}\right|<1\right\}$.

Demonstração. A demonstração feita a seguir vale da mesma forma para $X=c_{0}, \ell_{\infty}^{n}$ ou $\ell_{\infty}$.

Tome $k \in\left\{i \in \mathbb{N}:\left|x_{i}\right|<1\right\}$. Então

$$
\left\|x \pm\left(1-\left|x_{k}\right|\right) e_{k}\right\| \leq 1
$$


o que implica

$$
\left\|T\left(x \pm\left(1-\left|x_{k}\right|\right) e_{k}\right)\right\| \leq\|T\| .
$$

Afirmamos que $\left\|T\left(x \pm\left(1-\left|x_{k}\right|\right) e_{k}\right)\right\|=\|T\|$. De fato, como $\|T\| B_{Y}$ é convexo, se tivéssemos $\left\|T\left(x+\left(1-\left|x_{k}\right|\right) e_{k}\right)\right\|<\|T\|$ ou $\left\|T\left(x-\left(1-\left|x_{k}\right|\right) e_{k}\right)\right\|<\|T\|$, pela Proposição 1.1.9 deveria valer que

$$
\left\|\frac{1}{2} T\left(x+\left(1-\left|x_{k}\right|\right) e_{k}\right)+\frac{1}{2} T\left(x-\left(1-\left|x_{k}\right|\right) e_{k}\right)\right\|=\|T(x)\|<\|T\|,
$$

contrariando a hipótese. Logo,

$$
\frac{1}{\|T\|}\left\|T\left(x \pm\left(1-\left|x_{k}\right|\right) e_{k}\right)\right\|=1
$$

Suponhamos por absurdo que $T\left(e_{k}\right) \neq 0$. Em particular, $T\left(x+\left(1-\left|x_{k}\right|\right) e_{k}\right) \neq T\left(x-\left(1-\left|x_{k}\right|\right) e_{k}\right)$. Tendo em vista (3.2.1) e que $Y$ é estritamente convexo, vem

$$
\left\|\frac{1}{2\|T\|} T\left(x+\left(1-\left|x_{k}\right|\right) e_{k}\right)+\frac{1}{2\|T\|} T\left(x-\left(1-\left|x_{k}\right|\right) e_{k}\right)\right\|<1,
$$

isto é, $\|T(x)\|<\|T\|$, absurdo. Concluímos então que $T\left(e_{k}\right)=0$ para todo $k \in\left\{i \in \mathbb{N}:\left|x_{i}\right|<\right.$ $1\}$.

Lembramos que dados dois espaços normados $X$ e $Y, \mathcal{K}(X, Y)$ denota o espaço normado dos operadores lineares compactos definidos entre $X$ e $Y$, e $\mathcal{F}(X, Y)$ denota o espaço normado dos operadores lineares contínuos de posto finito definidos entre $X$ e $Y$.

Corolário 3.2.4. Se $Y$ é um espaço de Banach estritamente convexo, então $\mathcal{N} \mathcal{A}\left(c_{0}, Y\right) \subset \mathcal{F}\left(c_{0}, Y\right)$. Em particular, $\overline{\mathcal{N} \mathcal{A}\left(c_{0}, Y\right)} \subset \mathcal{K}\left(c_{0}, Y\right)$.

Demonstração. Considere $T \in \mathcal{N} \mathcal{A}\left(c_{0}, Y\right)$. Então existe $x \in S_{c_{0}}$ tal que $\|T(x)\|=\|T\|$. Como $x \in c_{0}$, existe $n_{0} \in \mathbb{N}$ tal que $\left|x_{n}\right|<1$ se $n>n_{0}$. Pela Proposição 3.2.3, concluímos que $T\left(e_{n}\right)=0$ para todo $n>n_{0}$. Com isso, mostramos que a imagem de $T$ está contida no subespaço gerado pelos vetores $T\left(e_{1}\right), \ldots, T\left(e_{n_{0}}\right)$. De fato, para todo $y \in c_{0}$,

$$
T(y)=T\left(\sum_{n=1}^{\infty} y_{n} e_{n}\right)=\sum_{n=1}^{\infty} y_{n} T\left(e_{n}\right)=\sum_{n=1}^{n_{0}} y_{n} T\left(e_{n}\right),
$$

e portanto $T \in \mathcal{F}\left(c_{0}, Y\right)$.

Como o fecho (em $\left.\mathcal{L}\left(c_{0}, Y\right)\right)$ do conjunto dos operadores de posto finito está contido em $\mathcal{K}\left(c_{0}, Y\right)$ (vide Proposição 1.1.28), segue que $\overline{\mathcal{N} \mathcal{A}\left(c_{0}, Y\right)} \subset \mathcal{K}\left(c_{0}, Y\right)$.

O Corolário abaixo é imediato:

Corolário 3.2.5. Se $Y$ é espaço de Banach estritamente convexo e existe um operador nãocompacto em $\mathcal{L}\left(c_{0}, Y\right)$, então $\mathcal{N} \mathcal{A}\left(c_{0}, Y\right)$ não é denso em $\mathcal{L}\left(c_{0}, Y\right)$.

Até então, denotamos por $c_{0}$ o espaço de Banach formado pelo espaço vetorial das sequências em $\mathbb{K}$ convergindo a 0 , munido da norma $\|.\|_{\infty}$. Entretanto, agora desejamos definir uma nova 
norma neste espaço. Para não carregar a notação, até o final desta seção denotaremos também por $c_{0}$ tal espaço vetorial. Não há perigo de confusão, pois cada significado será adotado no contexto adequado.

Feita essa ressalva, construiremos a seguir uma norma $\|\cdot\|_{K}$ em $c_{0}$, equivalente à $\|\cdot\|_{\infty}$, tal que o espaço normado $\left(c_{0},\|\cdot\|_{K}\right)$ seja estritamente convexo. Para facilitar nosso trabalho, definiremos antes uma norma auxiliar $\|.\|_{A}$ em $c_{0}$.

Afirmamos que a aplicação

$$
\begin{aligned}
<,>: c_{0} \times c_{0} & \rightarrow \mathbb{K} \\
(x, y) & \mapsto \sum_{i=1}^{\infty} \frac{x_{i} \overline{y_{i}}}{2^{i}}
\end{aligned}
$$

é um produto interno em $c_{0}$. Primeiro observemos que a aplicação está bem definida para qualquer par $(x, y) \in c_{0} \times c_{0}$, pois a série acima sempre converge absolutamente. É claro também que $<,>$ é linear na primeira coordenada e vale que $\langle x, y\rangle=\overline{\langle y, x\rangle}$ para todos $x, y \in c_{0}$, pela continuidade da conjugação. Por fim, $\langle x, x\rangle \geq 0$ para todo $x \in c_{0},\langle 0,0\rangle=0$ e se $\langle y, y\rangle=0$ para algum $y \in c_{0}$, então

$$
0=<y, y>=\sum_{i=1}^{\infty} \frac{\left|y_{i}\right|^{2}}{2^{i}} \geq \frac{\left|y_{i_{0}}\right|^{2}}{2^{i_{0}}} \geq 0 \text { para todo } i_{0} \in \mathbb{N},
$$

o que implica que $y=0$.

Logo, se $\|.\|_{A}$ for a norma induzida em $c_{0}$ pelo produto interno definido acima, $\left(c_{0},\|.\|_{A}\right)$ é um espaço com produto interno. Sendo assim, pela Proposição $1.4 .4,\left(c_{0},\|.\|{ }_{A}\right)$ é estritamente convexo. Entretanto, $\|\cdot\|_{A}$ e $\|\cdot\|_{\infty}$ não são equivalentes. De fato, vale que $e_{n} \stackrel{\|\cdot\|_{\mathrm{A}}}{\rightarrow} 0$ e $e_{n} \stackrel{\|\cdot\|_{\infty}}{\rightarrow} 0$.

Definamos agora a norma $\|.\| \|_{K}$ em $c_{0}$ dada por $\|x\|_{K} \doteq\|x\|_{\infty}+\|x\|_{A}$ para cada $x \in c_{0}$. Então:

Lema 3.2.6. A norma $\|.\|_{K}$ em $c_{0}$ é equivalente a $\|.\|_{\infty} e\left(c_{0},\|.\|_{K}\right)$ é um espaço de Banach estritamente convexo.

Demonstração. Vamos verificar primeiro que as normas $\|.\|_{\infty}$ e $\|.\|_{K}$ são equivalentes. Claramente, vale que $\|x\|_{K} \geq\|x\|_{\infty}$ para todo $x \in c_{0}$. Além disso, dado $x \in c_{0}$,

$$
\begin{aligned}
\|x\|_{A} & =\sqrt{\sum_{i=1}^{\infty} \frac{\left|x_{i}\right|^{2}}{2^{i}}} \\
& \leq \sqrt{\sum_{i=1}^{\infty} \frac{\|x\|_{\infty}^{2}}{2^{i}}} \\
& =\|x\|_{\infty} .
\end{aligned}
$$

Portanto, para todo $x \in c_{0},\|x\|_{K}=\|x\|_{\infty}+\|x\|_{A} \leq 2\|x\|_{\infty}$. Então temos

$$
\|x\|_{\infty} \leq\|x\|_{K} \leq 2\|x\|_{\infty} \text { para todo } x \in c_{0}
$$

e assim as normas $\|.\|_{K}$ e $\|.\|_{\infty}$ são equivalentes. Como $\left(c_{0},\|\cdot\|_{\infty}\right)$ é espaço de Banach, segue que $\left(c_{0},\|\cdot\|_{K}\right)$ é espaço de Banach. 
Mostraremos agora que $\left(c_{0},\|\cdot\|_{K}\right)$ é estritamente convexo. Para isso, faremos uso da caracterização de espaços estritamente convexos dada pela Proposição 1.4.3. Sejam $x, y \in c_{0}$ linearmente independentes. Como $\left(c_{0},\|.\|_{A}\right)$ é estritamente convexo, vale que

$$
\|x+y\|_{A}<\|x\|_{A}+\|y\|_{A} .
$$

Então

$$
\begin{aligned}
\|x+y\|_{K} & =\|x+y\|_{\infty}+\|x+y\|_{A} \\
& \leq\left(\|x\|_{\infty}+\|y\|_{\infty}\right)+\|x+y\|_{A} \\
& \stackrel{(3.2 .2)}{<}\left(\|x\|_{\infty}+\|y\|_{\infty}\right)+\left(\|x\|_{A}+\|y\|_{A}\right) \\
& =\|x\|_{K}+\|y\|_{K},
\end{aligned}
$$

e portanto $\left(c_{0},\|\cdot\|_{K}\right)$ é estritamente convexo.

Finalmente, estamos aptos a dar o exemplo pretendido. Lembremos que usamos a notação $c_{0}$ para o espaço vetorial das sequências em $\mathbb{K}$ convergentes a 0 e também para o espaço de Banach $\left(c_{0},\|\cdot\|_{\infty}\right)$, cada qual no contexto adequado.

Exemplo 3.2.7. Seja $Y \doteq\left(c_{0},\|.\|_{K}\right)$. Afirmamos que $\mathcal{N} \mathcal{A}\left(c_{0}, Y\right)$ não é denso em $\mathcal{L}\left(c_{0}, Y\right)$.

De fato, seja $I: c_{0} \rightarrow Y$ o operador identidade. Dados $n$ e $m$ naturais distintos, temos que

$$
\begin{aligned}
\left\|I\left(e_{n}\right)-I\left(e_{m}\right)\right\|_{K} & =\left\|e_{n}-e_{m}\right\|_{K} \\
& =\left\|e_{n}-e_{m}\right\|_{\infty}+\left\|e_{n}-e_{m}\right\|_{A} \\
& \geq 1 .
\end{aligned}
$$

Logo, $\left(e_{n}\right)_{n \in \mathbb{N}}$ é uma sequência limitada em $c_{0}$ tal que $\left(I\left(e_{n}\right)\right)_{n \in \mathbb{N}}$ não possui subsequência convergente em $Y$, o que implica que o operador $I$ não é compacto. Pelo Corolário 3.2.5, segue que $\mathcal{N} \mathcal{A}\left(c_{0}, Y\right)$ não é denso em $\mathcal{L}\left(c_{0}, Y\right)$.

\subsection{Propriedade de Bishop-Phelps-Bollobás para operadores}

Nesta seção estudaremos a extensão do Teorema de Bishop-Phelps-Bollobás a operadores. Para isso, definimos a Propriedade de Bishop-Phelps-Bollobás para operadores (BPBp), conceito introduzido por Acosta et al. no artigo [AAGM08], de 2008. Em seguida, apresentamos alguns resultados sobre tal propriedade, contidos principalmente em [AAGM08] e [AGKM17]. Exibimos, por exemplo, as demonstrações de [AAGM08] de que o par $(X, Y)$ satisfaz a BPBp se $X$ e $Y$ têm dimensão finita e de que $(X, Y)$ satisfaz a BPBp para todo espaço de Banach $X$, se $Y$ tem a propriedade $\beta$. Apresentamos também a classe de espaços de Banach $Y$ introduzida em [AGKM17] e a demonstração de que espaços contidos nesta classe são tais que $\left(c_{0}, Y\right)$ satisfaz a BPBp.

Definição 3.3.1. Sejam $X$ e $Y$ espaços de Banach. Dizemos que o par $(X, Y)$ satisfaz a Propriedade de Bishop-Phelps-Bollobás para operadores (BPBp) se dado $\epsilon>0$, existe $\eta(\epsilon)>0$ tal que para quaisquer $T \in S_{\mathcal{L}(X, Y)}$ e $x \in S_{X}$ satisfazendo a $\|T(x)\|>1-\eta(\epsilon)$ existem $u \in S_{X}$ e $S \in S_{\mathcal{L}(X, Y)}$ 
tais que

$$
\|S(u)\|=1,\|u-x\|<\epsilon \mathrm{e}\|S-T\|<\epsilon .
$$

Pelo Teorema de Bishop-Phelps-Bollobás e a discussão que o sucede, se $X$ é um espaço de Banach qualquer, tomando por exemplo

$$
\eta(\epsilon)= \begin{cases}\epsilon^{2} / 4, & \text { se } 0<\epsilon<1 \\ 1 / 16, & \text { se } \epsilon \geq 1\end{cases}
$$

obtemos que $(X, \mathbb{K})$ satisfaz a BPBp. Notemos ainda que no caso dos pares da forma $(X, \mathbb{K})$ a função $\eta$ definida não depende do espaço de Banach $X$ considerado, tratando-se de uma propriedade adicional. As conclusões anteriores continuam válidas trocando $\mathbb{K}$ por um espaço de Banach $Y$ de dimensão 1 sobre $\mathbb{K}$, já que todos os espaços dessa forma são isometricamente isomorfos.

Proposição 3.3.2. Seja $Y$ um espaço de Banach com $\operatorname{dim} Y=1$. Para todo espaço de Banach $X$, o $\operatorname{par}(X, Y)$ satisfaz a BPBp.

Notemos que se o par $(X, Y)$ satisfaz a BPBp, então $\mathcal{N} \mathcal{A}(X, Y)$ é denso em $\mathcal{L}(X, Y)$. Mais adiante nesta seção veremos que a recíproca não vale.

Observação 3.3.3. Vejamos que se $S \in S_{\mathcal{L}(X, Y)}$ e $\tilde{u}, \tilde{x} \in S_{X}$ forem tais que $\|S(\tilde{u})\|=1$ e $\|\tilde{x}-\tilde{u}\|<\epsilon$, definindo $u \doteq \lambda \tilde{u}$ e $x \doteq \lambda \tilde{x}$, com $\lambda \in S_{\mathbb{K}}$, teremos também que $\|S(u)\|=1$ e $\|x-u\|<\epsilon$.

Em particular, para que um par $(X, Y)$ satisfaça a BPBp basta que para dado $\epsilon>0$ exista $\eta(\epsilon)>0$ tal que sempre que $T \in S_{\mathcal{L}(X, Y)}$ e $x \in S_{X}$ forem tais que $\|T(x)\|>1-\eta(\epsilon)$, é possível encontrar $S \in S_{\mathcal{L}(X, Y)}$ e $\tilde{u} \in S_{X}$ que satisfazem a $\|S(\tilde{u})\|=1,\|S-T\|<\epsilon$ e $\|\tilde{u}-\tilde{x}\|<\epsilon$, para algum ponto $\tilde{x} \in S_{X}$ obtido por uma rotação de $x$, isto é, $\tilde{x}=\lambda x$, com $\lambda \in S_{\mathbb{K}}$. De fato, $u \doteq \lambda^{-1} \tilde{u}$ é tal que $\|S(u)\|=1$ e $\|u-x\|<\epsilon$.

Vimos na seção anterior que sempre que $X$ tem dimensão finita vale que $\mathcal{N} \mathcal{A}(X, Y)=\mathcal{L}(X, Y)$. Podemos garantir que o par $(X, Y)$ satisfaz a BPBp se $Y$ também tem dimensão finita, como mostra o seguinte resultado de [AAGM08].

Teorema 3.3.4 ([AAGM08]). Sejam $X$ e $Y$ espaços de Banach de dimensão finita. Para cada $\epsilon>0$, existe $\delta>0$ tal que para todo $T \in S_{\mathcal{L}(X, Y)}$, existe $R \in S_{\mathcal{L}(X, Y)}$ satisfazendo as seguintes condições:

(i) $\|R-T\|<\epsilon$.

(ii) Se $x \in S_{X}$ satisfaz a $\|T(x)\|>1-\delta$, então existe $\tilde{x} \in S_{X}$ tal que $\|R(\tilde{x})\|=1$ e $\|x-\tilde{x}\|<\epsilon$.

Demonstração. Vamos provar o resultado por absurdo. Suponhamos que para algum $\epsilon_{0}>0$ vale que para todo $\delta>0$ existe um operador em $S_{\mathcal{L}(X, Y)}$ que não satisfaz simultaneamente os itens (i)

e (ii) do enunciado para nenhum $R \in S_{\mathcal{L}(X, Y)}$. Para cada $\delta=\frac{1}{n}$, com $n \in \mathbb{N}$, seja $T_{n} \in S_{\mathcal{L}(X, Y)}$ tal operador.

Vale então que, para cada $n \in \mathbb{N}$ e $R \in S_{\mathcal{L}(X, Y)}$, se $\left\|R-T_{n}\right\|<\epsilon_{0}$, existe $x_{n, R} \in S_{X}$ satisfazendo a:

(a) $\left\|T_{n}\left(x_{n, R}\right)\right\|>1-\frac{1}{n}$. 
(b) $\left(\|R(\tilde{x})\|=1 \Rightarrow\left\|x_{n, R}-\tilde{x}\right\| \geq \epsilon_{0}\right)$, para todo $\tilde{x} \in S_{X}$.

Como $X$ e $Y$ têm dimensão finita, $\mathcal{L}(X, Y)$ tem dimensão finita, o que implica que $S_{\mathcal{L}(X, Y)}$ é compacto. Logo, $\left(T_{n}\right)_{n \in \mathbb{N}}$ tem uma subsequência $\left(T_{n_{k}}\right)_{k \in \mathbb{N}}$ convergente a algum $T_{0} \in S_{\mathcal{L}(X, Y)}$.

Tome $k_{0} \in \mathbb{N}$ tal que

$$
k \in \mathbb{N}, k \geq k_{0} \Rightarrow\left\|T_{n_{k}}-T_{0}\right\|<\epsilon_{0} .
$$

Definamos, para cada natural $k \geq k_{0}$, o ponto $x_{k} \doteq x_{n_{k}, T_{0}} \in S_{X}$. Como $S_{X}$ também é compacto, existe uma subsequência $\left(x_{k_{j}}\right)_{j \in \mathbb{N}}$ convergindo a algum $x_{0} \in S_{X}$. Observemos que $\left\|T_{n_{k_{j}}}-T_{0}\right\|<\epsilon_{0}$ para todo $j \in \mathbb{N}$, por construção. Portanto, pela letra (a), dado $j \in \mathbb{N}$ vale que

$$
1 \geq\left\|T_{n_{k_{j}}}\left(x_{k_{j}}\right)\right\|>1-\frac{1}{n_{k_{j}}}
$$

e assim tomando $j \rightarrow \infty$ obtemos $\left\|T_{0}\left(x_{0}\right)\right\|=1$. Temos também que para algum $j_{0} \in \mathbb{N}$ suficientemente grande, $\left\|x_{k_{j_{0}}}-x_{0}\right\|=\left\|x_{n_{k_{0}}, T_{0}}-x_{0}\right\|<\epsilon_{0}$. Isto é uma contradição, pois tendo em vista que $\left\|T_{n_{k_{0}}}-T_{0}\right\|<\epsilon_{0}$ e $\left\|T_{0}\left(x_{0}\right)\right\|=1$, o item (b) implica que $\left\|x_{n_{k_{0}}, T_{0}}-x_{0}\right\| \geq \epsilon_{0}$.

Corolário 3.3.5. Se $X$ e $Y$ são espaços de Banach de dimensão finita, o par $(X, Y)$ satisfaz a propriedade de Bishop-Phelps-Bollobás.

Notemos que o Teorema 3.3.4 corresponde a uma propriedade mais forte que a BPBp. De fato, ele afirma que para $X$ e $Y$ de dimensão finita, dado $\epsilon>0$, existe $\delta>0$ tal que se $T \in S_{\mathcal{L}(X, Y)}$, existe $R \in S_{\mathcal{L}(X, Y)}$ tal que $\|R-T\|<\epsilon$ e para qualquer $x \in S_{X}$ satisfazendo a $\|T(x)\|>1-\delta$, é possível encontrar $\tilde{x} \in S_{X}$ tal que $R$ atinge sua norma em $\tilde{x}$ e $\|x-\tilde{x}\|<\epsilon$. Aqui o $R \in S_{\mathcal{L}(X, Y)}$ encontrado depende apenas de $T$, e funciona para todo $x \in S_{X}$ obedecendo a $\|T(x)\|>1-\delta$.

Observação 3.3.6. Observemos que no caso em que $Y=\mathbb{K}$, a constante $\delta$ dada pelo Teorema 3.3.4 depende do espaço $X$, e não só de $\epsilon$, ao contrário do Teorema de Bishop-Phelps-Bollobás clássico.

A seguir apresentamos a demonstração de outro resultado de Acosta et al. em [AAGM08], que afirma se o espaço de Banach $Y$ tem a propriedade $\beta$ (vide Definição 3.1.10), então o par $(X, Y)$ satisfaz a BPBp para qualquer espaço de Banach $X$.

Teorema 3.3.7 ([AAGM08]). Sejam X e Y espaços de Banach. Se $Y$ tem a propriedade $\beta$, então o par $(X, Y)$ satisfaz a BPBp.

Demonstração. Sejam $\rho \in[0,1),\left\{f_{i}: i \in I\right\} \subset S_{Y^{*}}$ e $\left\{y_{i}: i \in I\right\} \subset S_{Y}$ a constante e os conjuntos que satisfazem às condições da propriedade $\beta$ para $Y$ (Definição 3.1.10). Vamos mostrar que $(X, Y)$ satisfaz a Propriedade de Bishop-Phelps-Bollobás para operadores com a função $\eta(\epsilon) \doteq \frac{\nu^{2}}{4}$, para $\nu$ escolhido de forma que $\nu<\frac{4 \epsilon}{15}(1-\rho)$, qualquer que seja $\epsilon \in(0,1)$.

Fixemos então $\epsilon \in(0,1)$, e tomemos $\nu<\frac{4 \epsilon}{15}(1-\rho)$. Pela escolha de $\nu$,

$$
\begin{aligned}
\nu+\frac{\nu^{2}}{4} & <\frac{4 \epsilon}{15}(1-\rho)+\frac{4 \epsilon^{2}}{15^{2}}(1-\rho)^{2} \\
& \leq \frac{64 \epsilon}{15^{2}}(1-\rho)
\end{aligned}
$$




$$
<\frac{\epsilon}{3}(1-\rho)
$$

e isso implica que $\rho\left(\nu+\frac{\nu^{2}}{4}\right)<\frac{\epsilon}{3}(1-\rho)$, ou seja,

$$
\rho\left(\frac{\epsilon}{3}+\nu+\frac{\nu^{2}}{4}\right)<\frac{\epsilon}{3} .
$$

Agora sejam $T \in S_{\mathcal{L}(X, Y)}$ e $x \in S_{X}$ satisfazendo a $\|T(x)\|>1-\frac{\nu^{2}}{4}$. Como $Y$ tem a propriedade $\beta$, vale que

$$
\|T(x)\|=\sup \left\{\left|f_{i}(T(x))\right|: i \in I\right\},
$$

então em particular existe $i_{0} \in I$ tal que

$$
\left|f_{i_{0}}(T(x))\right|>1-\frac{\nu^{2}}{4}
$$

Pela Observação 3.3.3, podemos sem perda de generalidade assumir que $\left|f_{i_{0}}(T(x))\right|=f_{i_{0}}(T(x))$, realizando uma rotação em $x \in S_{X}$ se necessário. Como também vale que $T^{t}\left(f_{i_{0}}\right) \in B_{X^{*}} \backslash\{0\}$, obtemos

$$
\frac{\nu^{2}}{4}>1-f_{i_{0}}(T(x)) \geq 1-\frac{T^{t}\left(f_{i_{0}}\right)(x)}{\left\|T^{t}\left(f_{i_{0}}\right)\right\|}
$$

Portanto, podemos aplicar o Teorema de Bishop-Phelps-Bollobás ao funcional $\frac{T^{t}\left(f_{i_{0}}\right)}{\left\|T^{t}\left(f_{i_{0}}\right)\right\|} \in S_{X^{*}}$ e ao ponto $x \in S_{X}$ para obter $f \in S_{X^{*}}$ e $u \in S_{X}$ satisfazendo a

$$
\left\|f-\frac{T^{t}\left(f_{i_{0}}\right)}{\left\|T^{t}\left(f_{i_{0}}\right)\right\|}\right\|<\nu,\|u-x\|<\nu \text { e } f(u)=1 .
$$

Em particular,

$$
\|\| T^{t}\left(f_{i_{0}}\right)\left\|f-T^{t}\left(f_{i_{0}}\right)\right\|<\left\|T^{t}\left(f_{i_{0}}\right)\right\| \nu \leq \nu
$$

Notemos ainda que $\| T^{t}\left(f_{i_{0}}\right)|| \geq\left|f_{i_{0}}(T(x))\right|>1-\frac{\nu^{2}}{4}$. Assim,

$$
\left\|f-T^{t}\left(f_{i_{0}}\right)\right\| \leq\|f-\| T^{t}\left(f_{i_{0}}\right)\|f\|+\|\| T^{t}\left(f_{i_{0}}\right)\left\|f-T^{t}\left(f_{i_{0}}\right)\right\|<\frac{\nu^{2}}{4}+\nu .
$$

Definiremos agora um operador cuja normalização será o operador que procuramos. Seja $\tilde{S} \in$ $\mathcal{L}(X, Y)$ dado por

$$
\tilde{S}(x) \doteq T(x)+\left[\left(1+\frac{\epsilon}{3}\right) f(x)-T^{t}\left(f_{i_{0}}\right)(x)\right] y_{i_{0}}, \text { para todo } x \in X .
$$


Então $\tilde{S}^{t} \in \mathcal{L}\left(Y^{*}, X^{*}\right)$ será dado por

$$
\tilde{S}(g)=T^{t}(g)+\left[\left(1+\frac{\epsilon}{3}\right) f-T^{t}\left(f_{i_{0}}\right)\right] g\left(y_{i_{0}}\right), \text { para todo } g \in Y^{*} .
$$

Novamente usando que $Y$ tem a propriedade $\beta$, pela Proposição 1.1.19 temos

$$
\|\tilde{S}\|=\left\|\tilde{S}^{t}\right\|=\sup _{j \in I}\left\|\tilde{S}^{t}\left(f_{j}\right)\right\|
$$

Em particular, $\left\|\tilde{S}^{t}\right\| \geq\left\|\tilde{S}^{t}\left(f_{i_{0}}\right)\right\|=1+\frac{\epsilon}{3}$, e se $j \in I \backslash\left\{i_{0}\right\}$

$$
\begin{aligned}
\left\|S^{t}\left(f_{j}\right)\right\| & =\left\|T^{t}\left(f_{j}\right)+\left[\left(1+\frac{\epsilon}{3}\right) f-T^{t}\left(f_{i_{0}}\right)\right] f_{j}\left(y_{i_{0}}\right)\right\| \\
& \leq 1+\rho\left\|\left(1+\frac{\epsilon}{3}\right) f-T^{t}\left(f_{i_{0}}\right)\right\| \\
& \leq 1+\rho\left(\left\|f-T^{t}\left(f_{i_{0}}\right)\right\|+\frac{\epsilon}{3}\right) \\
& \quad \leq 1+\rho\left(\nu+\frac{\nu^{2}}{4}+\frac{\epsilon}{3}\right) \\
& \stackrel{(3.3 .2)}{\leq}<1+\frac{\epsilon}{3} .
\end{aligned}
$$

De (3.3.3), concluímos que $\|\tilde{S}\|=\left\|\tilde{S}^{t}\left(f_{i_{0}}\right)\right\|=1+\frac{\epsilon}{3}$. Notemos ainda que como $\tilde{S}^{t}\left(f_{i_{0}}\right)=\left(1+\frac{\epsilon}{3}\right) f$, temos $\left(f_{i_{0}} \circ \tilde{S}\right)(u)=\left(1+\frac{\epsilon}{3}\right)$. Logo, $\tilde{S}$ atinge sua norma em $u \in S_{X}$. Definindo $S \doteq \frac{\tilde{S}}{\|\tilde{S}\|}$, obtemos que $S \in S_{\mathcal{L}(X, Y)}$ atinge sua norma em $u \in S_{X}$ e

$$
\begin{aligned}
\|S-T\| & \leq\|\tilde{S}-T\|+\|S-\tilde{S}\| \\
& =\left\|\left(1+\frac{\epsilon}{3}\right) f-T^{t}\left(f_{i_{0}}\right)\right\|+\frac{\epsilon}{3} \\
& \leq\left\|f-T^{t}\left(f_{i_{0}}\right)\right\|+\frac{2 \epsilon}{3} \\
& \stackrel{(3.3 .2)}{<} \nu+\frac{\nu^{2}}{4}+\frac{2 \epsilon}{3} .
\end{aligned}
$$

Novamente usando que $\nu+\frac{\nu^{2}}{4}<\frac{\epsilon}{3}(1-\rho) \leq \frac{\epsilon}{3}$, concluímos que $\|S-T\|<\epsilon$. Como já tínhamos também $\|x-u\|<\epsilon$, a demonstração está encerrada.

Como já discutido, no artigo [AAGM08], Acosta et al. introduziram a Propriedade de BishopPhelps-Bollobás para operadores e demonstraram os Teoremas 3.3.4 e 3.3.7 apresentados aqui. Neste artigo foi feita ainda uma caracterização dos espaços de Banach $Y$ tais que o par $\left(\ell_{1}, Y\right)$ satisfaz a 
BPBp, e provado que o par $\left(\ell_{\infty}^{n}, Y\right)$ satisfaz a BPBp se $Y$ for uniformemente convexo, para todo $n \in \mathbb{N}$.

Muitos outros trabalhos e resultados importantes sobre a BPBp foram produzidos desde então. Por exemplo, no caso real, Kim, Lee e Lin provaram que o par $\left(L_{\infty}, Y\right)$ satisfaz a BPBp sempre que $Y$ for uniformemente convexo [KLL16]. Para o caso complexo, Acosta mostrou que o par $\left(C_{0}(L), Y\right)$ satisfaz a BPBp para todo espaço uniformemente convexo $Y$ e todo espaço topológico Hausdorff localmente compacto $L$ [Aco16]. Existe também uma caracterização dos espaços de Banach $Y$ tais que o par $\left(\ell_{\infty}^{3}, Y\right)$ satisfaz a BPBp $\left[\mathrm{ABGG}^{+} 15\right]$, e uma para que $\left(\ell_{\infty}^{4}, Y\right)$ satisfaça a propriedade [ADSM19]. Entretanto, o caso do par $\left(c_{0}, Y\right)$ é diferente dos supracitados, e ainda não há uma caracterização dos pares $\left(c_{0}, Y\right)$ que satisfazem a BPBp. Veremos a seguir alguns resultados sobre este par.

Primeiramente, observemos que não é verdade que $\left(c_{0}, Y\right)$ satisfaz a BPBp para todo espaço de Banach $Y$. De fato, como estudado na seção anterior, $\mathcal{N} \mathcal{A}\left(c_{0}, Y\right)$ não é denso em $\mathcal{L}\left(c_{0}, Y\right)$ para $Y=\left(c_{0},\|\cdot\|_{K}\right)$ e, portanto, $\left(c_{0}, Y\right)$ não satisfaz a BPBp.

Em [AAGM08], após a demonstração de que $\left(\ell_{\infty}^{n}, Y\right)$ satisfaz a BPBp para todo $Y$ uniformemente convexo, ficou aberta a seguinte pergunta: o par $\left(c_{0}, Y\right)$ também satisfaz a BPBp se $Y$ for uniformemente convexo? Esta pergunta viria a ser respondida de forma afirmativa em 2012, por Kim [Kim13]. Em 2017, Acosta et al. generalizaram este resultado, apresentando uma classe maior de espaços de Banach $Y$ tais que $\left(c_{0}, Y\right)$ satisfaz a BPBp [AGKM17]. Apresentaremos ainda nesta seção tal classe, e estudaremos os resultados feitos em [AGKM17].

Para começar, vamos mostrar que se $\left(c_{0}, Y\right)$ satisfaz a BPBp para o espaço de Banach $Y$, então o mesmo valerá para os pares $\left(\ell_{\infty}^{n}, Y\right)$, para cada $n \in \mathbb{N}$. A demonstração de um resultado bem mais geral que este encontra-se em $\left[\mathrm{ACK}^{+} 15\right]$.

Observemos que se $T \in \mathcal{L}\left(\ell_{\infty}^{n}, Y\right)$ e $x \in \ell_{\infty}^{n}$, para algum $n \in \mathbb{N}$, podemos definir um operador $\tilde{T} \in \mathcal{L}\left(c_{0}, Y\right)$ por $\tilde{T}(y)=T\left(y_{1}, \ldots, y_{n}\right)$ para cada $y \in c_{0}$, e um ponto $\tilde{x} \doteq \sum_{i=1}^{n} x_{i} e_{i} \in c_{0}$. Vale que $\|\tilde{T}\|=\|T\|$ e $\|\tilde{x}\|=\|x\|$.

De forma análoga, se $\tilde{S} \in \mathcal{L}\left(c_{0}, Y\right)$ e $\tilde{u} \in c_{0}$, podemos definir um operador $S \in \mathcal{L}\left(\ell_{\infty}^{n}, Y\right)$ por $S(y)=\tilde{S}\left(\sum_{i=1}^{n} y_{i} e_{i}\right)$ para cada $y \in \ell_{\infty}^{n}$, e um ponto $u \doteq\left(\tilde{u}_{1}, \ldots, \tilde{u}_{n}\right) \in \ell_{\infty}^{n}$. Vale que $\|S\| \leq\|\tilde{S}\|$ e $\|u\| \leq\|\tilde{u}\|$.

Proposição 3.3.8 ([ACK $\left.\left.{ }^{+} 15\right]\right)$. Seja $Y$ um espaço de Banach. Se o par $\left(c_{0}, Y\right)$ satisfaz a BPBp, então $\left(\ell_{\infty}^{n}, Y\right)$ satisfaz a BPBp, para todo $n \in \mathbb{N}$.

Demonstraçãa. Fixemos $n \in \mathbb{N}$, e tomemos $\epsilon \in(0,1)$. Por hipótese, existe $\eta(\epsilon)>0$ que satisfaz as condições da BPBp para o par $\left(c_{0}, Y\right)$. Sejam $T \in S_{\mathcal{L}\left(\ell_{\infty}^{n}, Y\right)}$ e $x \in S_{\ell_{\infty}^{n}}$ satisfazendo a $\|T(x)\|>$ $1-\eta(\epsilon)$. Considere o operador $\tilde{T} \in S_{\mathcal{L}\left(c_{0}, Y\right)}$ e o ponto $\tilde{x} \in S_{c_{0}}$ definidos na discussão que precede o enunciado desta Proposição, ou seja, $\tilde{T}(y)=T\left(y_{1}, \ldots, y_{n}\right)$ para cada $y \in c_{0}$, e $\tilde{x} \doteq \sum_{i=1}^{n} x_{i} e_{i} \in c_{0}$. Teremos que

$$
\|\tilde{T}(\tilde{x})\|=\left\|T\left(\tilde{x}_{1}, \ldots, \tilde{x}_{n}\right)\right\|=\|T(x)\|>1-\eta(\epsilon) .
$$

Então existem $\tilde{S} \in S_{\mathcal{L}\left(c_{0}, Y\right)}$ e $\tilde{u} \in S_{c_{0}}$ satisfazendo a

$$
\|\tilde{S}(\tilde{u})\|=1,\|\tilde{S}-\tilde{T}\|<\epsilon \mathrm{e}\|\tilde{u}-\tilde{x}\|<\epsilon .
$$


Definindo

$$
\tilde{p} \doteq \sum_{i=1}^{n} \tilde{u}_{i} e_{i} \text { e } \tilde{q} \doteq \sum_{i=n+1}^{\infty} \tilde{u}_{i} e_{i}
$$

podemos escrever $\tilde{u}=\tilde{p}+\tilde{q}$, e $\operatorname{assim} 1=\|\tilde{u}\|=\operatorname{máx}\{\|\tilde{p}\|,\|\tilde{q}\|\}$. Por (3.3.4), $\|\tilde{x}-\tilde{p}\|<\epsilon$ e $\|\tilde{q}\|<\epsilon<1$. Em particular, $\|\tilde{p}\|=1$.

Consideremos agora o operador $S \in B_{\mathcal{L}\left(\ell_{\infty}^{n}, Y\right)}$ e o ponto $u \in B_{\ell_{\infty}^{n}}$ dados por $S(y)=\tilde{S}\left(\sum_{i=1}^{n} y_{i} e_{i}\right)$ para cada $y \in \ell_{\infty}^{n}$, e $u \doteq\left(\tilde{u}_{1}, \ldots, \tilde{u}_{n}\right)$, também definidos na discussão que precede o enunciado desta Proposição. Temos que

$$
\|S-T\| \leq\|\tilde{S}-\tilde{T}\|<\epsilon \text { e } \quad\|x-u\| \leq\|\tilde{x}-\tilde{u}\|<\epsilon .
$$

Resta mostrar que $\|S(u)\|=1$. Como $\|\tilde{q}\|<\epsilon$, segue que $\tilde{p}+\frac{\tilde{q}}{\epsilon} \in B_{c_{0}}$. Então

$$
\|\tilde{S}(\tilde{u})\|=\left\|(1-\epsilon) \tilde{S}(\tilde{p})+\epsilon \tilde{S}\left(\tilde{p}+\epsilon^{-1} \tilde{q}\right)\right\|=1,
$$

donde concluímos que

$$
1 \leq(1-\epsilon)\|\tilde{S}(\tilde{p})\|+\epsilon\left\|\tilde{S}\left(\tilde{p}+\epsilon^{-1} \tilde{q}\right)\right\| \leq(1-\epsilon)+\epsilon=1 .
$$

$\operatorname{Logo},\|\tilde{S}(\tilde{p})\|=\left\|\tilde{S}\left(\tilde{p}+\epsilon^{-1} \tilde{q}\right)\right\|=1$, e $\operatorname{assim}\|S(u)\|=\|\tilde{S}(\tilde{p})\|=1$.

De agora em diante, concentraremo-nos no estudo do artigo [AGKM17], onde é apresentada uma classe de espaços de Banach $Y$, a qual contêm os espaços uniformemente convexos e os que possuem a propriedade $\beta$ de Lindenstrauss, tais que $\left(c_{0}, Y\right)$ satisfaz a BPBp. Antes de apresentar esta classe e partir para o estudo dos teoremas relacionados, faremos alguns resultados que nos auxiliarão nessa tarefa.

O Lema abaixo, feito em [AAGM08], usa a noção de somabilidade, discutida na seção 1.6 do trabalho.

Lema 3.3.9 ([AAGM08]). Sejam $\left(c_{n}\right)_{n \in \mathbb{N}}$ uma sequência de números complexos tais que $\left|c_{n}\right| \leq 1$ para todo $n \in \mathbb{N}, \eta>0$ um número real e $\left(\alpha_{n}\right)_{n \in \mathbb{N}}$ uma sequência de reais não-negativos satisfazendo $a:$

(i) $\sum_{n=1}^{\infty} \alpha_{n} \leq 1$;

(ii) $\operatorname{Re} \sum_{n=1}^{\infty} \alpha_{n} c_{n}>1-\eta$.

Para $r \in(0,1)$, seja $A \doteq\left\{i \in \mathbb{N}: \operatorname{Re}\left(c_{i}\right)>r\right\}$. Então

$$
\sum_{n \in A} \alpha_{n}>1-\frac{\eta}{1-r}
$$

Demonstração. Para todo $n \in \mathbb{N}$, temos que $\left|\alpha_{n} \operatorname{Re}\left(c_{n}\right)\right| \leq \alpha_{n}$, e assim $\sum_{n=1}^{\infty}\left|\alpha_{n} \operatorname{Re}\left(c_{n}\right)\right|<\infty$. Pela Proposição 1.6.6, a família $\left(\alpha_{n} \operatorname{Re}\left(c_{n}\right)\right)_{n \in \mathbb{N}}$ é somável. Logo, também vale que $\sum_{n \in \mathbb{N}} \alpha_{n} \operatorname{Re}\left(c_{n}\right)=$ $\sum_{n=1}^{\infty} \alpha_{n} \operatorname{Re}\left(c_{n}\right)$, e pela Proposição 1.6.5 podemos escrever $\sum_{n=1}^{\infty} \alpha_{n} \operatorname{Re}\left(c_{n}\right)=\sum_{n \in A} \alpha_{n} \operatorname{Re}\left(c_{n}\right)+$ $\sum_{n \in A^{c}} \alpha_{n} \operatorname{Re}\left(c_{n}\right)$. Analogamente, $\sum_{n=1}^{\infty} \alpha_{n}=\sum_{n \in A} \alpha_{n}+\sum_{n \in A^{c}} \alpha_{n}$. 
Como já observado, $\alpha_{n} \operatorname{Re}\left(c_{n}\right) \leq \alpha_{n}$ para todo $n \in \mathbb{N}$, e portanto

$$
\sum_{n \in A} \alpha_{n} \operatorname{Re}\left(c_{n}\right) \leq \sum_{n \in A} \alpha_{n}
$$

(lembre-se de que a soma indexada em um conjunto de índices como definida na seção 1.6 é um limite de rede, que é monotônico). Por outro lado, se $n \notin A$, temos por definição $\operatorname{Re}\left(c_{n}\right) \leq r$, donde vem que

$$
\sum_{n \in A^{c}} \alpha_{n} \operatorname{Re}\left(c_{n}\right) \leq r \sum_{n \in A^{c}} \alpha_{n}
$$

Portanto, pela hipótese (ii) teremos

$$
\begin{aligned}
1-\eta & <\operatorname{Re} \sum_{n=1}^{\infty} \alpha_{n} c_{n} \\
& =\sum_{n=1}^{\infty} \alpha_{n} \operatorname{Re}\left(c_{n}\right) \\
& =\sum_{n \in A} \alpha_{n} \operatorname{Re}\left(c_{n}\right)+\sum_{n \in A^{c}} \alpha_{n} \operatorname{Re}\left(c_{n}\right) \\
& \leq \sum_{n \in A} \alpha_{n}+r \sum_{n \in A^{c}} \alpha_{n} .
\end{aligned}
$$

Como $\sum_{n=1}^{\infty} \alpha_{n}=\sum_{n \in A} \alpha_{n}+\sum_{n \in A^{c}} \alpha_{n} \leq 1$, vem

$$
1-\eta<\sum_{n \in A} \alpha_{n}+r\left(1-\sum_{n \in A} \alpha_{n}\right)=(1-r) \sum_{n \in A} \alpha_{n}+r .
$$

Concluímos então que $\sum_{n \in A} \alpha_{n}>1-\frac{\eta}{1-r}$.

Abaixo enunciamos a aplicação do Lema acima que usaremos para a apresentação dos resultados estudados.

Corolário 3.3.10. Sejam $f \in B_{c_{0} *}$ e $x \in B_{c_{0}}$ tais que $\operatorname{Re} f(x)>1-\eta$, para $0<\eta<1$. Para cada $n \in \mathbb{N}$, seja $\lambda_{n} \in S_{\mathbb{K}}$ satisfazendo a $f\left(e_{n}\right)=\lambda_{n}\left|f\left(e_{n}\right)\right|$. Então dado $r \in(0,1-\eta)$, o conjunto $A^{\prime} \doteq\left\{n \in \mathbb{N}: \operatorname{Re}\left(\lambda_{n} x_{n}\right)>r\right.$ e $\left.f\left(e_{n}\right) \neq 0\right\}$ é não-vazio, finito e satisfaz a

$$
\sum_{n \in A^{\prime}}\left|f\left(e_{n}\right)\right|>1-\frac{\eta}{1-r} .
$$

Demonstração. Suponhamos por absurdo que $A^{\prime}=\emptyset$. Então para todo $n \in \mathbb{N}$ vale que $\operatorname{Re}\left(x_{n} \lambda_{n}\right) \leq r$ ou $f\left(e_{n}\right)=0$. Em particular, para todo $n \in \mathbb{N}$ teremos que $\left|f\left(e_{n}\right)\right| \operatorname{Re}\left(x_{n} \lambda_{n}\right) \leq\left|f\left(e_{n}\right)\right| r$. Além disso, pela Proposição 1.1.14, $\|f\|=\sum_{n=1}^{\infty}\left|f\left(e_{n}\right)\right| \leq 1$. Assim,

$$
\begin{aligned}
\operatorname{Re} f(x) & =\operatorname{Re} f\left(\sum_{n=1}^{\infty} x_{n} e_{n}\right) \\
& =\operatorname{Re} \sum_{n=1}^{\infty} x_{n} \lambda_{n}\left|f\left(e_{n}\right)\right|
\end{aligned}
$$




$$
\begin{aligned}
& =\sum_{n=1}^{\infty}\left|f\left(e_{n}\right)\right| \operatorname{Re}\left(x_{n} \lambda_{n}\right) \\
& \leq r \sum_{n=1}^{\infty}\left|f\left(e_{n}\right)\right| \leq r,
\end{aligned}
$$

absurdo, pois por hipótese $\operatorname{Re} f(x)>1-\eta>r$. Então $A^{\prime} \neq \emptyset$.

Como $x \in c_{0}$, existe $n_{0} \in \mathbb{N}$ tal que $n>n_{0} \Rightarrow\left|x_{n}\right|<r$. Logo, $n>n_{0}$ implica que

$$
\operatorname{Re}\left(x_{n} \lambda_{n}\right) \leq\left|x_{n} \lambda_{n}\right|=\left|x_{n}\right|<r
$$

donde concluímos que $A^{\prime} \subset\left\{1, \ldots, n_{0}\right\}$, e portanto $A^{\prime}$ é finito.

Notemos agora que $\left(\lambda_{n} x_{n}\right)_{n \in \mathbb{N}}$ é sequência de complexos tais que $\left|\lambda_{n} x_{n}\right| \leq 1$ para todo $n \in \mathbb{N}$ e $\left(\left|f\left(e_{n}\right)\right|\right)_{n \in \mathbb{N}}$ é sequência de reais não-negativos satisfazendo a

$$
\sum_{n=1}^{\infty}\left|f\left(e_{n}\right)\right| \leq 1
$$

e

$$
\operatorname{Re} f(x)=\operatorname{Re} \sum_{n=1}^{\infty}\left|f\left(e_{n}\right)\right| x_{n} \lambda_{n}>1-\eta
$$

Se $A \doteq\left\{n \in \mathbb{N}: \operatorname{Re}\left(\lambda_{n} x_{n}\right)>r\right\}$, pelo Lema 3.3.9 temos que

$$
\sum_{n \in A^{\prime}}\left|f\left(e_{n}\right)\right|=\sum_{n \in A}\left|f\left(e_{n}\right)\right|>1-\frac{\eta}{1-r}
$$

Recordemos aqui que no final da seção 1.6 definimos o operador $P_{A} \in \mathcal{L}\left(c_{0}, c_{0}\right)$ por $P_{A}(x)=$ $\sum_{n \in A} x_{n} e_{n}$ para cada $x \in c_{0}$, para um dado $A \subset \mathbb{N}$. Vimos também que para todos $x \in c_{0}$ e $n \in \mathbb{N}$,

$$
\left(P_{A}(x)\right)_{n}= \begin{cases}x_{n}, & \text { se } n \in A \\ 0, & \text { se } n \notin A\end{cases}
$$

Antes de apresentar o seguinte Lema de [AGKM17], definemos para um dado $x \in c_{0}$ o conjunto supp $x \doteq\left\{n \in \mathbb{N}: x_{n} \neq 0\right\}$.

Lema 3.3.11 ([AGKM17]). Sejam $Y$ um espaço de Banach, $E \subset S_{Y}$ e $F: E \rightarrow S_{Y^{*}}$ tal que E é fortemente e uniformemente exposta por $F$. Suponhamos que para $\epsilon>0$, o real $\delta>0$ satisfaça a condição

$$
\left(y \in B_{Y}, e \in E, \operatorname{Re} F(e)(y)>1-\delta\right) \Rightarrow\|y-e\|<\epsilon
$$

Se $T \in S_{\mathcal{L}\left(c_{0}, Y\right)}$ e $x \in B_{c_{0}}$ são tais que $\operatorname{Re} F\left(e_{0}\right)(T(x))>1-\delta$ para algum $e_{0} \in E$ e $A \doteq$ supp $x$, então $\left\|T\left(I-P_{A}\right)\right\| \leq 2 \epsilon$.

Demonstração. Seja $u \in B_{c_{0}}$. Denotando por $I$ o operador identidade em $\mathcal{L}\left(c_{0}, c_{0}\right)$, temos que

$$
\left(\left(I-P_{A}\right)(u)\right)_{n}= \begin{cases}0, & \text { se } n \in A \\ u_{n}, & \text { se } n \notin A .\end{cases}
$$


Como $A=\operatorname{supp} x$, vale que $\left(x \pm\left(I-P_{A}\right)(u)\right) \in B_{c_{0}}$ e assim $T\left(x \pm\left(I-P_{A}\right)(u)\right) \in B_{Y}$. Escolhamos $s \in\{-1,1\}$ de forma que $\operatorname{Re} F\left(e_{0}\right)\left(s\left(I-P_{A}\right)(u)\right) \geq 0$. Como $\operatorname{Re} F\left(e_{0}\right)(T(x))>1-\delta$ por hipótese, segue que

$$
\left\|T(x)-e_{0}\right\|<\epsilon \operatorname{e} \operatorname{Re} F\left(e_{0}\right)\left(T\left(x+s\left(I-P_{A}\right)(u)\right)>1-\delta .\right.
$$

Portanto, $\left\|T\left(x+s\left(I-P_{A}\right)(u)\right)-e_{0}\right\|<\epsilon \mathrm{e}$

$$
\begin{aligned}
\left\|T\left(I-P_{A}\right)(u)\right\| & =\left\|s T\left(I-P_{A}\right)(u)\right\| \\
& =\left\|s T\left(I-P_{A}\right)(u)+T(x)-T(x)+e_{0}-e_{0}\right\| \\
& =\left\|T\left(s\left(I-P_{A}\right)(u)+x\right)-e_{0}+e_{0}-T(x)\right\| \\
& \leq\left\|T\left(s\left(I-P_{A}\right)(u)+x\right)-e_{0}\right\|+\left\|T(x)-e_{0}\right\| \\
& <2 \epsilon .
\end{aligned}
$$

Como $u \in B_{c_{0}}$ era arbitrário, $\left\|T\left(I-P_{A}\right)\right\| \leq 2 \epsilon$.

Seja $A \subset \mathbb{N}$. Definamos

$$
V_{A} \doteq\left\{x \in P_{A}\left(B_{c_{0}}\right):\left|x_{i}\right|=1 \text { para todo } i \in A\right\}
$$

Se $A$ tiver infinitos elementos, obrigatoriamente $V_{A}=\emptyset$, já que um elemento de $c_{0}$ não pode ter infinitas coordenadas de módulo 1 . Caso $A=\emptyset$, teremos $P_{A}=0$ e $V_{A}=\{0\}$. Portanto, esse conjunto só será interessante se $A$ for finito e não-vazio.

Se tivermos $A=\left\{n_{1}, \ldots, n_{k}\right\}$, com $k \geq 1$, a aplicação

$$
\begin{aligned}
J: P_{A}\left(c_{0}\right) & \rightarrow \ell_{\infty}^{k} \\
x & \mapsto\left(x_{n_{1}}, \ldots, x_{n_{k}}\right)
\end{aligned}
$$

é um isomorfismo isométrico. De fato, $J$ é claramente linear e sobrejetora, e como $P_{A}\left(c_{0}\right)=$ $\operatorname{span}\left[e_{n_{1}}, \ldots, e_{n_{k}}\right]$, também preserva normas. Notemos ainda que $B_{P_{A}\left(c_{0}\right)}=P_{A}\left(B_{c_{0}}\right)$ e portanto $J\left(P_{A}\left(B_{c_{0}}\right)\right)=B_{\ell_{\infty}^{k}}$. Além disso, $J\left(V_{A}\right)=\left\{y \in \ell_{\infty}^{k}:\left|y_{i}\right|=1\right.$ para todo $\left.i=1, \ldots, k\right\}$. Tendo em vista que a envoltória convexa de $\left\{y \in \ell_{\infty}^{k}:\left|y_{i}\right|=1\right.$ para todo $\left.i=1, \ldots, k\right\}$ é $B_{\ell_{\infty}^{k}}$, temos que $\operatorname{Env}\left(J\left(V_{A}\right)\right)=B_{\ell_{\infty}^{k}}$, e pela Proposição 1.1.13, $J^{-1}\left(\operatorname{Env}\left(J\left(V_{A}\right)\right)\right)=\operatorname{Env}\left(V_{A}\right)=J^{-1}\left(B_{\ell_{\infty}^{k}}\right)$, ou seja,

$$
\operatorname{Env}\left(V_{A}\right)=P_{A}\left(B_{c_{0}}\right)
$$

As notações e conclusões acima serão usadas em alguns resultados futuros.

Lema 3.3.12. Sejam $Y$ um espaço normado, $A \subset \mathbb{N}$ um conjunto finito e $T \in \mathcal{L}\left(c_{0}, Y\right)$. Então existe $x \in V_{A}$ tal que $\|T(x)\|=\sup \left\{\|T(y)\|: y \in P_{A}\left(B_{c_{0}}\right)\right\}$.

Demonstração. Se $A=\emptyset$, então $P_{A}=0, V_{A}=\{0\}$, e a conclusão é clara.

Suponhamos então que $A=\left\{n_{1}, \ldots, n_{k}\right\}$, com $k \geq 1$. Observemos que $V_{A}$ é compacto, pois $\left\{y \in \ell_{\infty}^{k}:\left|y_{i}\right|=1\right.$ para todo $\left.i=1, \ldots, k\right\}$ o é. Então existe $x \in V_{A}$ tal que $\|T(x)\|=\sup \{\|T(y)\|$ : $\left.y \in V_{A}\right\}$. É claro que

$$
\|T(x)\| \leq \sup \left\{\|T(y)\|: y \in P_{A}\left(B_{c_{0}}\right)\right\} .
$$


Definamos o conjunto

$$
C \doteq\left\{y \in P_{A}\left(B_{c_{0}}\right):\|T(y)\| \leq\|T(x)\|\right\} .
$$

Como $V_{A} \subset C$ e $C$ é convexo, temos que $\operatorname{Env}\left(V_{A}\right)=P_{A}\left(B_{c_{0}}\right) \subset C \subset P_{A}\left(B_{c_{0}}\right)$. Logo, $C=P_{A}\left(B_{c_{0}}\right)$ e assim $\|T(y)\| \leq\|T(x)\|$ para todo $y \in P_{A}\left(B_{c_{0}}\right)$. Por isso e por (3.3.5), $\|T(x)\|=\sup \{\|T(y)\|$ : $\left.y \in P_{A}\left(B_{c_{0}}\right)\right\}$.

Corolário 3.3.13. Sejam $Y$ um espaço normado e $T \in \mathcal{L}\left(c_{0}, Y\right)$ um operador tal que $T\left(B_{c_{0}}\right)=$ $T\left(P_{A}\left(B_{c_{0}}\right)\right)$, para $A \subset \mathbb{N}$ finito. Então existe $x \in V_{A}$ tal que $\|T\|=\|T(x)\|$. Em particular, $\|T\|=\sup \left\{\|T(y)\|: y \in V_{A}\right\}$.

Demonstração. Basta observar que nesse caso $\|T\|=\sup \left\{\|T(y)\|: y \in B_{c_{0}}\right\}=\sup \{\|T(y)\|: y \in$ $\left.P_{A}\left(B_{c_{0}}\right)\right\}$, e a conclusão segue do Lema 3.3.12.

Suponhamos que $Y$ é um espaço de Banach tal que existem um conjunto $I,\left\{y_{i}: i \in I\right\} \subset S_{Y}$, $\left\{f_{i}: i \in I\right\} \subset S_{Y^{*}}$, um subconjunto $E \subset S_{Y}$, uma função $F: E \rightarrow S_{Y^{*}}$ e $\rho \in[0,1)$ satisfazendo a:

(I) $f_{i}\left(y_{i}\right)=1$, para todo $i \in I$.

(II) $\left|f_{i}\left(y_{j}\right)\right| \leq \rho$, para todos $i, j \in I, i \neq j$.

(III) $E$ é fortemente e uniformemente exposta por $F$.

(IV) $\left|F(e)\left(y_{i}\right)\right| \leq \rho$, para todos $e \in E$ e $i \in I$.

(V) O conjunto $G \doteq F(E) \cup\left\{f_{i}: i \in I\right\}$ é tal que, para todo $y \in Y$,

$$
\|y\|=\sup \{|f(y)|: f \in G\}=\max \left\{\sup \left\{\left|f_{i}(y)\right|: i \in I\right\}, \sup \{|F(e)(y)|: e \in E\}\right\} .
$$

O resultado principal do artigo [AGKM17] afirma que espaços de Banach $Y$ satisfazendo as condições acima são tais que o par $\left(c_{0}, Y\right)$ satisfaz a BPBP. Antes de apresentar este resultado, vamos fazer uma discussão preliminar a respeito de espaços de Banach satisfazendo a tais condições.

Fixemos $0<\epsilon<1$. Por (III), existem constantes $\nu>0$ e $\delta>0$ (que podemos escolher satisfazendo também a $\nu<\frac{\epsilon}{12}$ e $\delta<1$ ) tais que

$$
\left(e \in E, y \in B_{Y}, \operatorname{Re} F(e)(y)>1-\nu\right) \Rightarrow\|y-e\|<\frac{\epsilon}{8}
$$

e

$$
\left(e \in E, y \in B_{Y}, \operatorname{Re} F(e)(y)>1-\delta\right) \Rightarrow\|y-e\|<\frac{\nu^{3}}{8} .
$$

Definamos ainda $\epsilon_{1} \doteq \frac{\delta \nu^{3}}{16}$ e fixemos $\eta, s \in(0,1)$ satisfazendo a

$$
\begin{array}{r}
\eta<\frac{4}{5} \min \left\{\frac{\epsilon_{1}(1-\rho)}{(1+\rho)}, \frac{\epsilon}{3}, 2 \delta\right\} ; \\
s<\min \left\{\frac{\epsilon^{2} \epsilon_{1}\left(\delta-2 \epsilon_{1}\right)}{24\left(1+2 \epsilon_{1}\right)}, \frac{\eta^{2} \epsilon_{1} \epsilon^{2}}{3 \cdot 2^{5}}\right\} .
\end{array}
$$


Notemos que todas as contantes definidas dependem do $\epsilon>0$ fixado no início.

Fixada a notação acima, listaremos algumas desigualdades envolvendo as constantes que serão usadas futuramente. Indicaremos quando cada desigualdade for usada, de modo que a lista pode ser consultada aos poucos, conforme surja necessidade.

Desigualdade 1: $\rho\left(\eta+\frac{\eta^{2}}{4}+\epsilon_{1}\right)<\epsilon_{1}$.

Prova. Por escolha de $\eta$, temos que $\eta<\frac{4 \epsilon_{1}}{5} \frac{(1-\rho)}{(1+\rho)}$. Logo,

$$
\frac{\eta^{2}}{4}<\frac{4 \epsilon_{1}^{2}}{25} \frac{(1-\rho)^{2}}{(1+\rho)^{2}}
$$

Como $\rho \in[0,1)$ e $\epsilon_{1} \in(0,1)$, obtemos

$$
\begin{aligned}
\eta+\frac{\eta^{2}}{4} & <\frac{4 \epsilon_{1}}{5} \frac{(1-\rho)}{(1+\rho)}+\frac{4 \epsilon_{1}^{2}}{25} \frac{(1-\rho)^{2}}{(1+\rho)^{2}} \\
& \leq \frac{4 \epsilon_{1}}{5}(1-\rho)+\frac{4 \epsilon_{1}}{25}(1-\rho) \\
& =\frac{24 \epsilon_{1}}{25}(1-\rho)<\epsilon_{1}(1-\rho),
\end{aligned}
$$

donde segue que $\rho\left(\eta+\frac{\eta^{2}}{4}\right) \leq\left(\eta+\frac{\eta^{2}}{4}\right)<\epsilon_{1}(1-\rho)$ e portanto $\rho\left(\eta+\frac{\eta^{2}}{4}+\epsilon_{1}\right)<\epsilon_{1}$.

Desigualdade 2: $2 \epsilon_{1}+\eta+\frac{\eta^{2}}{4}<\epsilon$.

Prova. Vimos na demonstração da Desigualdade 1 que $\eta+\frac{\eta^{2}}{4}<\frac{24 \epsilon_{1}}{25}(1-\rho)<\epsilon_{1}$. Como $3 \epsilon_{1}=$ $\frac{3 \delta}{16} \nu^{3}<\nu^{3}<\epsilon^{3}<\epsilon$, concluímos que $2 \epsilon_{1}+\eta+\frac{\eta^{2}}{4}<3 \epsilon_{1}<\epsilon$.

Desigualdade 3: $\frac{8 s}{\epsilon^{2}}<\delta$.

Prova. Por construção, $s<\frac{\epsilon^{2} \epsilon_{1}\left(\delta-2 \epsilon_{1}\right)}{24\left(1+2 \epsilon_{1}\right)}$, e então $\frac{8 s}{\epsilon^{2}}<\frac{\epsilon_{1}\left(\delta-2 \epsilon_{1}\right)}{3\left(1+2 \epsilon_{1}\right)}<\delta$.

Desigualdade 4: $2 \epsilon_{1}+\eta+\frac{\eta^{2}}{4}+\frac{\nu^{3}}{4}<\epsilon$.

Prova. Como visto na demonstração da Desigualdade $2,2 \epsilon_{1}+\eta+\frac{\eta^{2}}{4}<3 \epsilon_{1}$. Mas $3 \epsilon_{1}=\frac{3 \delta}{16} \nu^{3}<\frac{\nu^{3}}{4}$.

Portanto, $2 \epsilon_{1}+\eta+\frac{\eta^{2}}{4}+\frac{\nu^{3}}{4}<\frac{\nu^{3}}{4}+\frac{\nu^{3}}{4}<\nu^{3}<\epsilon^{3}<\epsilon$.

Desigualdade 5: $1-\frac{\eta^{2}}{4}<\left(1-\frac{8 s}{\epsilon^{2}}\right)^{2}-\frac{8 s}{\epsilon^{2} \epsilon_{1}}$. 
Prova. Por construção, $\frac{8 s}{\epsilon^{2}}<\frac{\eta^{2} \epsilon_{1}}{12}<\frac{\eta^{2}}{12}$, e então

$$
1-\frac{\eta^{2}}{12}<1-\frac{8 s}{\epsilon^{2}}
$$

Notemos que para todo $x \in \mathbb{R}$ vale que $(1-2 x) \leq(1-x)^{2}$. Para $x=\frac{\eta^{2}}{12}$, vem

$$
1-\frac{\eta^{2}}{6} \leq\left(1-\frac{\eta^{2}}{12}\right)^{2}
$$

Pelas desigualdades destacadas acima, obtemos $1-\frac{\eta^{2}}{6}<\left(1-\frac{8 s}{\epsilon^{2}}\right)^{2}$ e, novamente pela escolha de $s, \frac{8 s}{\epsilon^{2} \epsilon_{1}}<\frac{\eta^{2}}{12}$. Logo,

$$
1-\frac{\eta^{2}}{6}+\frac{8 s}{\epsilon^{2} \epsilon_{1}}<\left(1-\frac{8 s}{\epsilon^{2}}\right)^{2}+\frac{\eta^{2}}{12}
$$

e, portanto,

$$
1-\frac{\eta^{2}}{4}<\left(1-\frac{8 s}{\epsilon^{2}}\right)^{2}-\frac{8 s}{\epsilon^{2} \epsilon_{1}}
$$

Desigualdade 6: $\left(1-\frac{8 s}{\epsilon^{2}}\right)^{2} \epsilon_{1}+\left(1-\frac{8 s}{\epsilon^{2}}\right)>1+\epsilon_{1}\left(1-\frac{\eta^{2}}{4}\right)$.

Prova. Da Desigualdade 5, obtemos imediatamente $\epsilon_{1}\left(1-\frac{\eta^{2}}{4}\right)<\epsilon_{1}\left(1-\frac{8 s}{\epsilon^{2}}\right)^{2}-\frac{8 s}{\epsilon^{2}}$ e, portanto, $1+\epsilon_{1}\left(1-\frac{\eta^{2}}{4}\right)<\left(1-\frac{8 s}{\epsilon^{2}}\right)^{2} \epsilon_{1}+\left(1-\frac{8 s}{\epsilon^{2}}\right)$

Desigualdade 7: $2 \epsilon_{1}+\frac{\eta^{2}}{4}<\delta$.

Prova. Por construção, $2 \epsilon_{1}=\frac{\delta \nu^{3}}{8}<\frac{\delta}{8}$ e $\frac{\eta}{2}<\frac{4 \delta}{5}$. Portanto, $\frac{\eta^{2}}{4}<\frac{16 \delta^{2}}{25}<\frac{16 \delta}{25}$ e $2 \epsilon_{1}+\frac{\eta^{2}}{4}<$ $\left(\frac{1}{8}+\frac{16}{25}\right) \delta<\delta$

Desigualdade 8: $\frac{3 \nu}{4}<\frac{\nu}{1+\nu}$.

Prova. Por construção, $\nu<\frac{1}{3}$. A seguinte sequência de implicações nos dará a desigualdade desejada:

$$
\begin{aligned}
\nu<\frac{1}{3} & \Rightarrow \nu^{2}<\frac{\nu}{3} \\
& \Rightarrow 3 \nu^{2}+3 \nu<4 \nu
\end{aligned}
$$




$$
\Rightarrow \frac{3 \nu}{4}<\frac{\nu}{1+\nu}
$$

Desigualdade 9: $\frac{3 \nu}{2}+2 \epsilon_{1}<\frac{\epsilon}{2}$.

Prova. Temos que $2 \epsilon_{1}=\frac{\delta \nu^{3}}{8}<\frac{\nu}{2}$. Portanto, $\frac{3 \nu}{2}+2 \epsilon_{1}<2 \nu<2 \frac{\epsilon}{12}<\frac{\epsilon}{2}$.

Mantendo ainda as condições e notação acima fixadas, faremos um Lema. Observemos que a sua demonstração é bastante similar à do Teorema 3.3.7, e de fato as suas hipóteses de certa forma reproduzem o que ocorre quando $Y$ tem a propriedade $\beta$, que veremos ser um caso particular dos espaços satisfazendo às condições (I)-(V).

Lema 3.3.14. Nas condiçôes dadas acima, se existem $i_{0} \in I, \tilde{T} \in B_{\mathcal{L}\left(c_{0}, Y\right)}$ e $\tilde{z} \in S_{c_{0}}$ satisfazendo $a\left|f_{i_{0}}(\tilde{T}(\tilde{z}))\right|>1-\frac{\eta^{2}}{4}$, então existem $S \in S_{\mathcal{L}\left(c_{0}, Y\right)}$ e $z \in S_{c_{0}}$ tais que

$$
\|z-\tilde{z}\|<\eta,\|S-\tilde{T}\|<2 \epsilon_{1}+\eta+\frac{\eta^{2}}{4} e\|S(z)\|=1 .
$$

Demonstração. Pela Observação 3.3.3, podemos demonstrar o Lema substituindo z̃ por uma rotação qualquer deste ponto. Suponhamos então, sem perda de generalidade, que $f_{i_{0}}(\tilde{T}(\tilde{z}))=\left|f_{i_{0}}(\tilde{T}(\tilde{z}))\right|$. Logo,

$$
\frac{\eta^{2}}{4}>1-f_{i_{0}}(\tilde{T}(\tilde{z}))=1-\tilde{T}^{t}\left(f_{i_{0}}\right)(\tilde{z})
$$

Em particular, temos ainda que $\left\|\tilde{T}^{t}\left(f_{i_{0}}\right)\right\|>1-\frac{\eta^{2}}{4}$. Como $\tilde{T}^{t}\left(f_{i_{0}}\right) \in B_{c_{0} *}$, obtemos

$$
\frac{\eta^{2}}{4}>\left|1-\frac{\tilde{T}^{t}\left(f_{i_{0}}\right)(\tilde{z})}{\left\|\tilde{T}^{t}\left(f_{i_{0}}\right)\right\|}\right| .
$$

Pelo Teorema de Bishop-Phelps-Bollobás, existem $g \in S_{c_{0}^{*}}$ e $z \in S_{c_{0}}$ tais que

$$
g(z)=1,\|\tilde{z}-z\|<\eta \text { e }\left\|g-\frac{\tilde{T}^{t}\left(f_{i_{0}}\right)}{\left\|\tilde{T}^{t}\left(f_{i_{0}}\right)\right\|}\right\|<\eta .
$$

Assim, $\|g\| \tilde{T}^{t}\left(f_{i_{0}}\right)\left\|-\tilde{T}\left(f_{i_{0}}\right)\right\|<\eta\left\|\tilde{T}^{t}\left(f_{i_{0}}\right)\right\| \leq \eta$ e então

$$
\left\|g-\tilde{T}^{t}\left(f_{i_{0}}\right)\right\| \leq\|g\| \tilde{T}^{t}\left(f_{i_{0}}\right)\left\|-\tilde{T}^{t}\left(f_{i_{0}}\right)\right\|+\|g\| \tilde{T}^{t}\left(f_{i_{0}}\right)\|-g\|<\eta+\frac{\eta^{2}}{4} .
$$

Definamos agora um operador $\tilde{S} \in \mathcal{L}\left(c_{0}, Y\right)$ dado por

$$
\tilde{S}(x) \doteq \tilde{T}(x)+\left[\left(1+\epsilon_{1}\right) g(x)-\tilde{T}^{t}\left(f_{i_{0}}\right)(x)\right] y_{i_{0}}, \text { para todo } x \in c_{0} .
$$

O operador transposto $\tilde{S}^{t} \in \mathcal{L}\left(Y^{*}, c_{0}^{*}\right)$ será dado por

$$
\tilde{S}^{t}(f)=\tilde{T}^{t}(f)+f\left(y_{i_{0}}\right)\left[\left(1+\epsilon_{1}\right) g-\tilde{T}^{t}\left(f_{i_{0}}\right)\right] \text {, para todo } f \in Y^{*} .
$$


Vamos estudar a norma de $\tilde{S}$. Como $Y$ obedece à condição (V) dada anteriormente, pela Proposição 1.1 .19 vale que

$$
\|\tilde{S}\|=\left\|\tilde{S}^{t}\right\|=\operatorname{máx}\left\{\sup \left\{\left|\tilde{S}^{t}\left(f_{i}\right)\right|: i \in I\right\}, \sup \left\{\left|\tilde{S}^{t}(F(e))\right|: e \in E\right\}\right\}
$$

Em particular, $\left\|\tilde{S}^{t}\right\| \geq\left\|\tilde{S}^{t}\left(f_{i_{0}}\right)\right\|=1+\epsilon_{1}$, e se $f \in F(E) \cup\left\{f_{i}: i \in I \backslash\left\{i_{0}\right\}\right\}$, pelas condições (II) e (IV) obtemos

$$
\begin{aligned}
\left\|\tilde{S}^{t}(f)\right\| & =\left\|\tilde{T}^{t}(f)+f\left(y_{i_{0}}\right)\left[\left(1+\epsilon_{1}\right) g-\tilde{T}^{t}\left(f_{i_{0}}\right)\right]\right\| \\
& \leq\left\|\tilde{T}^{t}(f)\right\|+\rho\left\|\left(1+\epsilon_{1}\right) g-\tilde{T}^{t}\left(f_{i_{0}}\right)\right\| \\
& \leq 1+\rho\left(\left\|g-\tilde{T}^{t}\left(f_{i_{0}}\right)\right\|+\epsilon_{1}\right) \\
& \stackrel{(3.3 .9)}{\leq} 1+\rho\left(\eta+\frac{\eta^{2}}{4}+\epsilon_{1}\right) \\
& \stackrel{\text { Des.1 }}{<} 1+\epsilon_{1} .
\end{aligned}
$$

Portanto, (3.3.10) implica que $\|\tilde{S}\|=\left\|\tilde{S}^{t}\right\|=\left\|\tilde{S}^{t}\left(f_{i_{0}}\right)\right\|=1+\epsilon_{1}$. Como $\tilde{S}^{t}\left(f_{i_{0}}\right)=\left(1+\epsilon_{1}\right) g$, vem que $f_{i_{0}}(\tilde{S}(z))=1+\epsilon_{1}$. Assim, $\|\tilde{S}(z)\|=1+\epsilon_{1}$, ou seja, $\tilde{S}$ atinge sua norma em $z \in S_{c_{0}}$. Logo, o operador $S \doteq \frac{\tilde{S}}{\|\tilde{S}\|} \in S_{\mathcal{L}\left(c_{0}, Y\right)}$ também atinge sua norma em $z$, e além disso

$$
\begin{aligned}
\|S-\tilde{T}\| & \leq\|S-\tilde{S}\|+\|\tilde{S}-\tilde{T}\| \\
& =|1-\|\tilde{S}\||+\left\|\left[\left(1+\epsilon_{1}\right) g-\tilde{T}^{t}\left(f_{i_{0}}\right)\right] y_{i_{0}}\right\| \\
& =\epsilon_{1}+\left\|\left(1+\epsilon_{1}\right) g-\tilde{T}^{t}\left(f_{i_{0}}\right)\right\| \\
& \leq 2 \epsilon_{1}+\left\|g-\tilde{T}^{t}\left(f_{i_{0}}\right)\right\| \\
& \stackrel{(3.3 .9)}{<} 2 \epsilon_{1}+\eta+\frac{\eta^{2}}{4} .
\end{aligned}
$$

Como já tínhamos que $\|\tilde{z}-z\|<\eta$, a demonstração está encerrada.

Apresentaremos em seguida o Teorema de [AGKM17] que afirma que se $Y$ for um espaço de Banach obedecendo às condições $(\mathrm{I})-(\mathrm{V})$ dadas anteriormente, o par $\left(c_{0}, Y\right)$ satisfaz a BPBp. Tais condições serão listadas novamente no enunciado, para maior clareza.

Teorema 3.3.15 ([AGKM17]). Seja $Y$ um espaço de Banach tal que existem um conjunto I, $\left\{y_{i}: i \in I\right\} \subset S_{Y},\left\{f_{i}: i \in I\right\} \subset S_{Y^{*}}$, um subconjunto $E \subset S_{Y}$, uma função $F: E \rightarrow S_{Y^{*}} e$ $\rho \in[0,1)$ satisfazendo a:

(I) $f_{i}\left(y_{i}\right)=1$, para todo $i \in I$.

(II) $\left|f_{i}\left(y_{j}\right)\right| \leq \rho$, para todos $i, j \in I, i \neq j$.

(III) E é fortemente e uniformemente exposta por F.

(IV) $\left|F(e)\left(y_{i}\right)\right| \leq \rho$, para todos e $\in E$ e $i \in I$. 
(V) O conjunto $G \doteq F(E) \cup\left\{f_{i}: i \in I\right\}$ é tal que, para todo $y \in Y$,

$$
\|y\|=\sup \{|f(y)|: f \in G\}=\max \left\{\sup \left\{\left|f_{i}(y)\right|: i \in I\right\}, \sup \{|F(e)(y)|: e \in E\}\right\} .
$$

Então o par $\left(c_{0}, Y\right)$ satisfaz a Propriedade de Bishop-Phelps-Bollobás para operadores.

Demonstração. Seja $\epsilon \in(0,1)$. Considere as constantes $\nu, \delta, \eta, s$ e $\epsilon_{1}$ construídas a partir do $\epsilon$ fixado, conforme a discussão feita anteriormente. Suponha que $T \in S_{\mathcal{L}\left(c_{0}, Y\right)}$ e $x_{0} \in S_{c_{0}}$ sejam tais que $\left\|T\left(x_{0}\right)\right\|>1-s$. Mostraremos que existem $S \in S_{\mathcal{L}\left(c_{0}, Y\right)}$ e $u \in S_{c_{0}}$ satisfazendo a $\|S(u)\|=1$, $\|S-T\|<\epsilon$ e $\left\|u-x_{0}\right\|<\epsilon$, donde a conclusão seguirá. Separaremos a demonstração em dois casos, e o segundo caso consistirá de dois subcasos.

Primeiro Caso. Existe $i \in I$ tal que $\left|f_{i}\left(T\left(x_{0}\right)\right)\right|>1-\frac{\eta^{2}}{4}$.

Notemos que para este caso estão satisfeitas as condições do Lema 3.3.14, e portanto existem $S \in S_{\mathcal{L}\left(c_{0}, Y\right)}$ e $u \in S_{c_{0}}$ satisfazendo a

$$
\|S(u)\|=1,\|S-T\|<2 \epsilon_{1}+\eta+\frac{\eta^{2}}{4} \text { e }\left\|u-x_{0}\right\|<\eta .
$$

Por construção, $\eta<\epsilon$, e pela Desigualdade $2,2 \epsilon_{1}+\eta+\frac{\eta^{2}}{4}<\epsilon$. Então $\|S-T\|<\epsilon$ e $\left\|u-x_{0}\right\|<\epsilon$, o que encerra a demonstração deste caso.

Segundo Caso. Para todo $i \in I,\left|f_{i}\left(T\left(x_{0}\right)\right)\right| \leq 1-\frac{\eta^{2}}{4}$.

Este caso é composto de dois subcasos. Antes de enunciá-los, serão definidos alguns elementos auxiliares e feitos alguns resultados que os envolvem.

Por contrução, $s<\frac{\eta^{2} \epsilon_{1} \epsilon^{2}}{3 \cdot 2^{5}}<\frac{\eta^{2}}{4}$, e assim $\left\|T\left(x_{0}\right)\right\|>1-s>1-\frac{\eta^{2}}{4}$. Pela hipótese $(\mathrm{V})$,

$$
\left\|T\left(x_{0}\right)\right\|=\max \left\{\sup \left\{\left|f_{i}\left(T\left(x_{0}\right)\right)\right|: i \in I\right\}, \sup \left\{\left|F(e)\left(T\left(x_{0}\right)\right)\right|: e \in E\right\}\right\}
$$

e como $\left|f_{i}\left(T\left(x_{0}\right)\right)\right| \leq 1-\frac{\eta^{2}}{4}$ para todo $i \in I$, existe $e_{0} \in E$ tal que $\left|F\left(e_{0}\right)\left(T\left(x_{0}\right)\right)\right|>1-s$. Definamos $f \doteq F\left(e_{0}\right) \in S_{Y^{*}}$. Tendo em vista a Observação 3.3.3, podemos assumir que $f\left(T\left(x_{0}\right)\right)=\left|f\left(T\left(x_{0}\right)\right)\right|$, fazendo uma rotação em $x_{0}$ se necessário.

Para cada $n \in \mathbb{N}$, fixemos $\lambda_{n} \in S_{\mathbb{K}}$ satisfazendo a $T^{t}(f)\left(e_{n}\right)=\lambda_{n}\left|T^{t}(f)\left(e_{n}\right)\right|$, e definamos

$$
A \doteq\left\{n \in \mathbb{N}: T^{t}(f)\left(e_{n}\right) \neq 0 \text { e } \operatorname{Re}\left(x_{0 n} \lambda_{n}\right)>1-\frac{\epsilon^{2}}{8}\right\}
$$

Como $T^{t}(f) \in B_{c_{0}^{*}}, \operatorname{Re} T^{t}(f)\left(x_{0}\right)=T^{t}(f)\left(x_{0}\right)>1-s$ e, por construção, $1-\frac{\epsilon^{2}}{8}<1-\frac{\epsilon^{2}}{8}\left(\frac{\eta^{2} \epsilon_{1}}{3 \cdot 2^{2}}\right)<$ $1-s$, pelo Corolário 3.3 .10 concluímos que $A$ é não-vazio, finito e

$$
\sum_{k \in A}\left|T^{t}(f)\left(e_{k}\right)\right|>1-\frac{s}{\epsilon^{2} / 8}=1-\frac{8 s}{\epsilon^{2}}
$$


Agora definamos uma sequência $a \in \mathbb{K}^{\mathbb{N}}$ por

$$
a_{k}= \begin{cases}\overline{\lambda_{k}}, & \text { se } k \in A \\ x_{0 k}, & \text { se } k \notin A .\end{cases}
$$

Como $A$ é finito e $x_{0} \in S_{c_{0}}$, temos que $a \in S_{c_{0}}$. Seja também $a_{1} \doteq P_{A}(a) \in S_{c_{0}}$, isto é,

$$
a_{1 k}= \begin{cases}\overline{\lambda_{k}}, & \text { se } k \in A \\ 0, & \text { se } k \notin A .\end{cases}
$$

Notemos que

$$
\begin{aligned}
T^{t}(f)\left(a_{1}\right) & =T^{t}(f)\left(\sum_{k \in A} \overline{\lambda_{k}} e_{k}\right) \\
& =\sum_{k \in A} \lambda_{k}^{-1} T^{t}(f)\left(e_{k}\right) \\
& =\sum_{k \in A} \lambda_{k}^{-1} \lambda_{k}\left|T^{t}(f)\left(e_{k}\right)\right| \\
& =\sum_{k \in A}\left|T^{t}(f)\left(e_{k}\right)\right| .
\end{aligned}
$$

Em particular, $T^{t}(f)\left(a_{1}\right) \in \mathbb{R}_{\geq 0}$. Pela Desigualdade 3 e (3.3.11), obtemos

$$
1-\delta<1-\frac{8 s}{\epsilon^{2}}<\operatorname{Re} T^{t}(f)\left(a_{1}\right)=\left|T^{t}(f)\left(a_{1}\right)\right| \leq\left\|T\left(a_{1}\right)\right\| .
$$

Observemos agora que se $a, b \in \mathbb{R}$ são tais que $|a+i b| \leq 1$, então $a^{2}+b^{2} \leq 1$ e $|1-(a+i b)|^{2}=$ $(1-a)^{2}+b^{2}=1-2 a+a^{2}+b^{2} \leq 2(1-a)$. Logo, $|1-(a+i b)| \leq \sqrt{2(1-a)}$. Em outras palavras,

$$
\lambda \in B_{\mathbb{K}} \Rightarrow|1-\lambda| \leq \sqrt{2(1-\operatorname{Re}(\lambda))}
$$

Usaremos este fato mais de uma vez no decorrer da demonstração.

Como $A$ é finito, temos que

$$
\| a-x_{0}||=\sup _{k \in \mathbb{N}}\left|a_{k}-x_{0 k}\right|=\max _{k \in A}\left|\overline{\lambda_{k}}-x_{0 k}\right|=\max _{k \in A}\left|1-\lambda_{k} x_{0 k}\right|,
$$

e como $\left|\lambda_{k} x_{0 k}\right| \leq 1$ para todo $k \in \mathbb{N}$, por (3.3.13) vale que $\left|1-\lambda_{k} x_{0 k}\right| \leq \sqrt{2\left(1-\operatorname{Re}\left(\lambda_{k} x_{0 k}\right)\right)}$ para todo $k \in \mathbb{N}$. Se $k \in A$, por construção $\operatorname{Re}\left(\lambda_{k} x_{0 k}\right)>1-\frac{\epsilon^{2}}{8}$, e então

$$
\frac{\epsilon}{2}>\sqrt{2\left(1-\operatorname{Re}\left(\lambda_{k} x_{0 k}\right)\right)} \text { para todo } k \in A
$$

o que implica que

$$
|| a-x_{0}||<\frac{\epsilon}{2} .
$$

Para finalizar esta discussão inicial do segundo caso, observemos que por (3.3.12), $\operatorname{Re} T^{t}(f)\left(a_{1}\right)=$ 
$\operatorname{Re} F\left(e_{0}\right)\left(T\left(a_{1}\right)\right)>1-\delta$, e vale também que $A=\operatorname{supp} a_{1}$. Devido a (3.3.7) e ao Lema 3.3.11, obtemos, respectivamente,

$$
\left\|T\left(a_{1}\right)-e_{0}\right\|<\frac{\nu^{3}}{8}
$$

e

$$
\left\|T-T P_{A}\right\| \leq \frac{\nu^{3}}{4}
$$

Consideremos agora os dois subcasos.

Subcaso 2.1. Existe $i \in I$ tal que $\left|f_{i}\left(T\left(a_{1}\right)\right)\right|>1-\frac{\eta^{2}}{4}$.

Seja $i_{0} \in I$ o índice que satisfaz a $\left|f_{i_{0}}\left(T\left(a_{1}\right)\right)\right|>1-\frac{\eta^{2}}{4}$. Como $P_{A}(a)=a_{1}$, temos que

$$
\left|f_{i_{0}}\left(\left(T P_{A}\right)(a)\right)\right|>1-\frac{\eta^{2}}{4},
$$

$\operatorname{com} T P_{A} \in B_{\mathcal{L}\left(c_{0}, Y\right)}$. Pelo Lema 3.3.14, existem $S \in S_{\mathcal{L}\left(c_{0}, Y\right)}$ e $u \in S_{c_{0}}$ satisfazendo a $\|u-a\|<\eta$ $\left\|S-T P_{A}\right\|<2 \epsilon_{1}+\eta+\frac{\eta^{2}}{4}$ e $\|S(u)\|=1$. Assim, teremos que

$$
\begin{gathered}
\|S-T\| \leq\left\|S-T P_{A}\right\|+\left\|T P_{A}-T\right\| \\
\stackrel{(3.3 .16)}{<} 2 \epsilon_{1}+\eta+\frac{\eta^{2}}{4}+\frac{\nu^{3}}{4} \\
\stackrel{\text { Des.4 }}{<} .
\end{gathered}
$$

Além disso,

$$
\begin{gathered}
\left\|u-x_{0}\right\| \leq\|u-a\|+\left\|a-x_{0}\right\| \\
\stackrel{(3.3 .14)}{<\eta+\frac{\epsilon}{2}<\epsilon}
\end{gathered}
$$

o que encerra a demonstração deste subcaso.

Subcaso 2.2. Para todo $i \in I,\left|f_{i}\left(T\left(a_{1}\right)\right)\right| \leq 1-\frac{\eta^{2}}{4}$.

Definamos o operador $\tilde{S} \in \mathcal{L}\left(c_{0}, Y\right)$ por

$$
\tilde{S}(x) \doteq T\left(P_{A}(x)\right)+\epsilon_{1} f\left(T\left(P_{A}(x)\right)\right) T\left(a_{1}\right) \text {, para todo } x \in c_{0} .
$$

Notemos que $\tilde{S} \neq 0$, já que $\tilde{S}(a)=T\left(a_{1}\right)+\epsilon_{1} f\left(T\left(a_{1}\right)\right) T\left(a_{1}\right)=\left(1+\epsilon_{1} f\left(T\left(a_{1}\right)\right)\right) T\left(a_{1}\right)$ e $\left|\epsilon_{1} f\left(T\left(a_{1}\right)\right)\right|<$ 1. Além disso, como $P_{A} P_{A}=P_{A}$, vale que $\tilde{S}=\tilde{S} P_{A}$.

Definamos ainda $S \doteq \frac{\tilde{S}}{\|\tilde{S}\|}$. Então $S P_{A}=S$ e em particular $S\left(P_{A}\left(B_{c_{0}}\right)\right)=S\left(B_{c_{0}}\right)$. Pelo Corolário 3.3.13, existe $a_{2} \in V_{A}\left(=\left\{x \in P_{A}\left(B_{c_{0}}\right):\left|x_{i}\right|=1\right.\right.$ para todo $\left.\left.i \in A\right\}\right)$ tal que $\|S\|=\left\|S\left(a_{2}\right)\right\|$. Como $V_{A}$ é invariante por rotação, isto é, $\lambda V_{A} \subset V_{A}$ para todo $\lambda \in S_{\mathbb{K}}$, podemos assumir sem perda de generalidade que $f\left(T\left(a_{2}\right)\right)=\left|f\left(T\left(a_{2}\right)\right)\right|$. Então teremos

$$
\left\|S\left(a_{2}\right)\right\|=\|S\|=1 \text { e } f\left(T\left(a_{2}\right)\right)=\left|f\left(T\left(a_{2}\right)\right)\right|
$$


e

$$
P_{A}\left(a_{2}\right)=a_{2} \text { e }\left|a_{2 k}\right|=1 \text { para todo } k \in A .
$$

Agora notemos que pela hipótese (V),

$$
1=|| S\left(a_{2}\right)||=\sup \left\{\left|h\left(S\left(a_{2}\right)\right)\right|: h \in G\right\} .
$$

Portanto, existe uma sequência $\left(h_{n}\right)_{n \in \mathbb{N}}$ em $G \subset B_{Y^{*}}$ tal que $\left|h_{n}\left(S\left(a_{2}\right)\right)\right| \rightarrow 1$. Pelo Teorema de Banach-Alaoglu, $B_{Y^{*}}$ é $w^{*}$-compacta, então existe uma subrede $\left(h_{n_{\mu}}\right)_{\mu \in M}$ convergente em $B_{Y^{*}}$, digamos $h_{n_{\mu}} \stackrel{w^{*}}{\rightarrow} h \in B_{Y^{*}}$. Em particular, $h_{n_{\mu}}\left(S\left(a_{2}\right)\right) \rightarrow h\left(S\left(a_{2}\right)\right)$, o que implica que $\left|h_{n_{\mu}}\left(S\left(a_{2}\right)\right)\right| \rightarrow$ $\left|h\left(S\left(a_{2}\right)\right)\right|$ e, portanto, $\left|h\left(S\left(a_{2}\right)\right)\right|=1$. Seja $\sigma \in S_{\mathbb{K}}$ tal que $(\sigma h)\left(S\left(a_{2}\right)\right)=\left|h\left(S\left(a_{2}\right)\right)\right|=1$, e definamos $g \doteq \sigma h$. Como a rede $\left(\sigma h_{n_{\mu}}\right)_{\mu \in M}$ em $S_{\mathbb{K}} G$ converge a $\sigma h=g \in B_{Y^{*}}$ na topologia $w^{*}$, temos que

$$
g \in{\overline{S_{\mathbb{K}} G}}^{w^{*}}={\overline{S_{\mathbb{K}} F(E) \cup S_{\mathbb{K}}\left\{f_{i}: i \in I\right\}}}^{w^{*}}={\overline{S_{\mathbb{K}} F(E)}}^{w^{*}} \cup \overline{S_{\mathbb{K}}\left\{f_{i}: i \in I\right\}}{ }^{*} .
$$

Além disso, $g\left(S\left(a_{2}\right)\right)=\sigma h\left(S\left(a_{2}\right)\right)=1$, donde concluímos que

$$
g\left(S\left(a_{2}\right)\right)=\left\|S\left(a_{2}\right)\right\|=1
$$

Agora seja $M$ o operador em $\mathcal{L}\left(c_{0}, c_{0}\right)$ dado por

$$
M(x)=\left(a_{2 n} \overline{a_{1 n}} x_{n}\right)_{n \in \mathbb{N}}, \text { para todo } x \in c_{0}
$$

Notemos que $M \in B_{\mathcal{L}\left(c_{0}, c_{0}\right)}$ e portanto $S M \in B_{\mathcal{L}\left(c_{0}, Y\right)}$. O nosso objetivo será mostrar que o operador $S M$ e o ponto $a \in S_{c_{0}}$ satisfazem a $\left\|a-x_{0}\right\|<\epsilon,\|S M-T\|<\epsilon$ e $\|(S M)(a)\|=1$. Observe que, por (3.3.14), já temos que $\left\|a-x_{0}\right\|<\epsilon$. Para mostrar o restante, precisamos de outras desigualdades e resultados, que serão feitos no que segue.

Vamos primeiro avaliar a norma de $\tilde{S}$. Temos que $\tilde{S}\left(a_{1}\right)=T\left(a_{1}\right)+\epsilon_{1} f\left(T\left(a_{1}\right)\right) T\left(a_{1}\right)$, e então

$$
f\left(\tilde{S}\left(a_{1}\right)\right)=f\left(T\left(a_{1}\right)\right)+\epsilon_{1}\left(f\left(T\left(a_{1}\right)\right)\right)^{2} .
$$

Por $(3.3 .12), \operatorname{Re} f\left(T\left(a_{1}\right)\right)=f\left(T\left(a_{1}\right)\right)>1-\frac{8 s}{\epsilon^{2}}$, o que implica

$$
\begin{aligned}
\|\tilde{S}\| & \geq\left|f\left(\tilde{S}\left(a_{1}\right)\right)\right| \\
& =\left|f\left(T\left(a_{1}\right)\right)+\epsilon_{1}\left(f\left(T\left(a_{1}\right)\right)\right)^{2}\right| \\
& =\left|f\left(T\left(a_{1}\right)\right)\right|+\epsilon_{1}\left|f\left(T\left(a_{1}\right)\right)\right|^{2} \\
& \geq 1-\frac{8 s}{\epsilon^{2}}+\epsilon_{1}\left(1-\frac{8 s}{\epsilon^{2}}\right)^{2} .
\end{aligned}
$$

Utilizando a Desigualdade 6, concluímos que

$$
\|\tilde{S}\| \geq 1-\frac{8 s}{\epsilon^{2}}+\epsilon_{1}\left(1-\frac{8 s}{\epsilon^{2}}\right)^{2}>1 .
$$


Por outro lado, para todo $x \in c_{0},\|\tilde{S}(x)\|=\left\|T\left(P_{A}(x)\right)+\epsilon_{1} f\left(T\left(P_{A}(x)\right)\right) T\left(a_{1}\right)\right\| \leq\left(1+\epsilon_{1}\right)\|x\|$ e então

$$
1<\|\tilde{S}\| \leq 1+\epsilon_{1}
$$

A seguir partiremos para o estudo de outras desigualdades.

Temos que $\left\|S-T P_{A}\right\| \leq\|S-\tilde{S}\|+\left\|\tilde{S}-T P_{A}\right\|=|1-\|\tilde{S}\||+\left\|\tilde{S}-T P_{A}\right\|$ e $\tilde{S}(x)-\left(T P_{A}\right)(x)=$ $\epsilon_{1} f\left(T\left(P_{A}(x)\right)\right) T\left(a_{1}\right)$ para todo $x \in c_{0}$. Logo, $\left\|\tilde{S}-T P_{A}\right\| \leq \epsilon_{1}$, donde obtemos

$$
\left\|S-T P_{A}\right\| \leq|1-\|\tilde{S}\||+\left\|\tilde{S}-T P_{A}\right\| \stackrel{(3.3 .21)}{\leq 2} \epsilon_{1}
$$

e, por (3.3.16),

$$
\|S-T\| \leq\left\|S-T P_{A}\right\|+\left\|T P_{A}-T\right\| \leq 2 \epsilon_{1}+\frac{\nu^{3}}{4} .
$$

Agora, por (3.3.19), $\operatorname{Re} g\left(\tilde{S}\left(a_{2}\right)\right)=\operatorname{Re} g\left(\|\tilde{S}\| S\left(a_{2}\right)\right)=\|\tilde{S}\|$, e por construção $f\left(T\left(a_{2}\right)\right) \in \mathbb{R}$ (vide (3.3.17)) e $P_{A}\left(a_{2}\right)=a_{2}$ (vide (3.3.18)). Logo,

$$
\begin{aligned}
\|\tilde{S}\| & =\operatorname{Re} g\left(\tilde{S}\left(a_{2}\right)\right) \\
& =\operatorname{Re} g\left(T\left(a_{2}\right)\right)+\operatorname{Re} g\left(\epsilon_{1} f\left(T\left(a_{2}\right)\right) T\left(a_{1}\right)\right) \\
& =\operatorname{Re} g\left(T\left(a_{2}\right)\right)+\epsilon_{1} \operatorname{Re} g\left(T\left(a_{1}\right)\right) \operatorname{Re} f\left(T\left(a_{2}\right)\right) \\
& \leq 1+\epsilon_{1} \operatorname{Re} g\left(T\left(a_{1}\right)\right) \operatorname{Re} f\left(T\left(a_{2}\right)\right) .
\end{aligned}
$$

Pela desigualdade acima e (3.3.20), obtemos:

$$
\begin{aligned}
& 1-\frac{8 s}{\epsilon^{2}}+\epsilon_{1}\left(1-\frac{8 s}{\epsilon^{2}}\right)^{2} \leq 1+\epsilon_{1} \operatorname{Re} f\left(T\left(a_{2}\right)\right) ; \\
& 1-\frac{8 s}{\epsilon^{2}}+\epsilon_{1}\left(1-\frac{8 s}{\epsilon^{2}}\right)^{2} \leq 1+\epsilon_{1} \operatorname{Re} g\left(T\left(a_{1}\right)\right) .
\end{aligned}
$$

Utilizando a Desigualdade 5, concluímos que

$$
\left|f\left(T\left(a_{2}\right)\right)\right|=\operatorname{Re} f\left(T\left(a_{2}\right)\right)>1-\frac{\eta^{2}}{4}
$$

e

$$
\left|g\left(T\left(a_{1}\right)\right)\right| \geq \operatorname{Re} g\left(T\left(a_{1}\right)\right)>1-\frac{\eta^{2}}{4} .
$$

Como já visto, $g \in \overline{S_{\mathbb{K}} F(E)} w^{*} \cup \overline{S_{\mathbb{K}}\left\{f_{i}: i \in I\right\}} w^{*}$. Afirmamos que $g \in \overline{S_{\mathbb{K}} F(E)} w^{*}$. De fato,

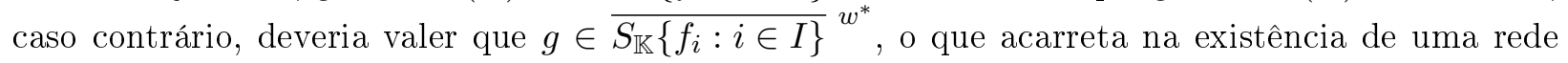
$\left(g_{\lambda}\right)_{\lambda \in \Lambda}$ em $S_{\mathbb{K}}\left\{f_{i}: i \in I\right\}$ tal que $g_{\lambda} \stackrel{w^{*}}{\rightarrow} g$. Em particular, teríamos que $g_{\lambda}\left(T\left(a_{1}\right)\right) \rightarrow g\left(T\left(a_{1}\right)\right)$ e, devido a (3.3.25), deveria existir $\lambda_{1} \in \Lambda$ tal que $\left|g_{\lambda_{1}}\left(T\left(a_{1}\right)\right)\right|>1-\frac{\eta^{2}}{4}$. Mas $g_{\lambda_{1}}=\chi f_{i_{1}}$, para certos $\chi \in S_{\mathbb{K}}$ e $i_{1} \in I$, o que implica $\left|f_{i_{1}}\left(T\left(a_{1}\right)\right)\right|>1-\frac{\eta^{2}}{4}$, contrariando a hipótese deste subcaso. Portanto, $g \in \overline{S_{\mathbb{K}} F(E)} w^{*}$. 
Avaliaremos agora $\operatorname{Re} g\left(S\left(a_{1}\right)\right)$. Para isso, notemos que

$$
\begin{aligned}
\left\|S-T P_{A}\right\| & \geq\left\|\left(S-T P_{A}\right)\left(a_{2}\right)\right\| \\
& =\left\|T\left(a_{2}\right)-S\left(a_{2}\right)\right\| \\
& \geq\left|f\left(T\left(a_{2}\right)-S\left(a_{2}\right)\right)\right| \\
& \geq \operatorname{Re} f\left(T\left(a_{2}\right)-S\left(a_{2}\right)\right),
\end{aligned}
$$

o que implica $\operatorname{Re} f\left(S\left(a_{2}\right)\right) \geq \operatorname{Re} f\left(T\left(a_{2}\right)\right)-\left\|S-T P_{A}\right\|$, e junto com (3.3.22) e (3.3.24) nos dá

$$
\operatorname{Re} f\left(S\left(a_{2}\right)\right)>1-\frac{\eta^{2}}{4}-2 \epsilon_{1} \stackrel{\text { Des.7 }}{>} 1-\delta
$$

ou seja, $\operatorname{Re} F\left(e_{0}\right)\left(S\left(a_{2}\right)\right)>1-\delta$. Por $(3.3 .7)$, concluímos que $\left\|S\left(a_{2}\right)-e_{0}\right\|<\frac{\nu^{3}}{8}$. Usando também (3.3.15),

$$
\left\|S\left(a_{2}\right)-T\left(a_{1}\right)\right\| \leq\left\|S\left(a_{2}\right)-e_{0}\right\|+\left\|e_{0}-T\left(a_{1}\right)\right\|<\frac{\nu^{3}}{4} .
$$

Observemos que $\operatorname{Re} g\left(S\left(a_{1}\right)\right)=\operatorname{Re} g\left(S\left(a_{2}\right)\right)+\operatorname{Re} g\left(S\left(a_{1}\right)-S\left(a_{2}\right)\right) \stackrel{(3.3 .19)}{=} 1-\operatorname{Re} g\left(S\left(a_{2}\right)-S\left(a_{1}\right)\right)$, donde vem

$$
\begin{aligned}
\operatorname{Re} g\left(S\left(a_{1}\right)\right) & =1-\operatorname{Re} g\left(S\left(a_{2}\right)-S\left(a_{1}\right)\right) \\
& \geq 1-\left|g\left(S\left(a_{2}\right)-S\left(a_{1}\right)\right)\right| \\
& \geq 1-\left\|S\left(a_{2}\right)-S\left(a_{1}\right)\right\| \\
& \geq 1-\left\|S\left(a_{2}\right)-T\left(a_{1}\right)\right\|-\left\|T\left(a_{1}\right)-S\left(a_{1}\right)\right\| .
\end{aligned}
$$

Usando (3.3.26) e que

$$
\left\|S\left(a_{1}\right)-T\left(a_{1}\right)\right\|=\left\|S\left(a_{1}\right)-\left(T P_{A}\right)\left(a_{1}\right)\right\| \leq\left\|S-T P_{A}\right\| \stackrel{(3.3 .22)}{\leq 2} \epsilon_{1},
$$

obtemos finalmente

$$
\operatorname{Re} g\left(S\left(a_{1}\right)\right)>1-2 \epsilon_{1}-\frac{\nu^{3}}{4}=1-\frac{\delta \nu^{3}}{8}-\frac{\nu^{3}}{4}>1-\frac{3 \nu^{3}}{8} .
$$

Faremos agora uma construção análoga àquela feita no início da demonstração do segundo caso. Para cada $k \in \mathbb{N}$, fixe $s_{k} \in S_{\mathbb{K}}$ satisfazendo a $S^{t}(g)\left(e_{k}\right)=s_{k}\left|S^{t}(g)\left(e_{k}\right)\right|$. Como $S^{t}(g)\left(a_{2}\right)=1$ e $a_{2}$ pertence à imagem de $P_{A}$, vem

$$
\begin{aligned}
1=S^{t}(g)\left(a_{2}\right) & =\sum_{k=1}^{\infty} a_{2 k} S^{t}(g)\left(e_{k}\right) \\
& =\sum_{k=1}^{\infty} a_{2 k} s_{k}\left|S^{t}(g)\left(e_{k}\right)\right| \\
& =\sum_{k \in A} a_{2 k} s_{k}\left|S^{t}(g)\left(e_{k}\right)\right| .
\end{aligned}
$$


Como $S^{t}(g) \in B_{c_{0}^{*}}$, temos que $\sum_{k=1}^{\infty}\left|S^{t}(g)\left(e_{k}\right)\right| \leq 1$. Logo, $1=\left|\sum_{k \in A} a_{2 k} s_{k}\right| S^{t}(g)\left(e_{k}\right)|| \leq \sum_{k \in A}\left|S^{t}(g)\left(e_{k}\right)\right| \leq$ 1, o que implica $\sum_{k \in A}\left|S^{t}(g)\left(e_{k}\right)\right|=1$. Em particular, $S^{t}(g)\left(e_{k}\right)=0$ quando $k \notin A$. Assim, temos:

(i) $1=\sum_{k \in A}\left|S^{t}(g)\left(e_{k}\right)\right|=\sum_{k \in A} s_{k} a_{2 k}\left|S^{t}(g)\left(e_{k}\right)\right|$;

(ii) $\left|s_{k} a_{2 k}\right|=1$ para todo $k \in A$;

(iii) $A$ é finito.

Em decorrência do Corolário 1.1.5, as afirmações dadas nos itens acima implicam que sempre que $k \in A$ for tal que $\left|S^{t}(g)\left(e_{k}\right)\right| \neq 0$, teremos $s_{k} a_{2 k}=1$, ou seja, $s_{k}=\overline{a_{2 k}}$. Assim,

$$
k \in A \text { e } S^{t}(g)\left(e_{k}\right) \neq 0 \Rightarrow s_{k}=\overline{a_{2 k}} .
$$

Definamos

$$
B \doteq\left\{k \in \mathbb{N}: S^{t}(g)\left(e_{k}\right) \neq 0 \text { e } \operatorname{Re}\left(s_{k} a_{1 k}\right)>1-\frac{\nu^{2}}{2}\right\} .
$$

Temos $B \subset A$, já que $S^{t}(g)\left(e_{k}\right)=0$ quando $k \notin A$. O nosso objetivo seguinte é avaliar $\operatorname{Re} g\left(S\left(P_{B}\left(a_{1}\right)\right)\right)$.

Como $\nu \in(0,1)$, vale que $\frac{3 \nu^{3}}{8}<\frac{\nu^{2}}{2}$. Tendo em vista a afirmação anterior e (3.3.27), podemos usar o Corolário 3.3.10 para concluir que $B$ é finito, não-vazio e satisfaz a

$$
\sum_{j \in B}\left|S^{t}(g)\left(e_{j}\right)\right|>1-\frac{3 \nu^{3} / 8}{\nu^{2} / 2}=1-\frac{3 \nu}{4} .
$$

Notemos agora que

$$
\begin{aligned}
\operatorname{Re} g\left(S\left(P_{B}\left(a_{1}\right)\right)\right) & =\operatorname{Re} g\left(\sum_{j \in B} a_{1 j} S\left(e_{j}\right)\right) \\
& =\operatorname{Re} \sum_{j \in B} a_{1 j} S^{t}(g)\left(e_{j}\right) \\
& =\operatorname{Re} \sum_{j \in B} a_{1 j} s_{j}\left|S^{t}(g)\left(e_{j}\right)\right| \\
& =\sum_{j \in B}\left|S^{t}(g)\left(e_{j}\right)\right| \operatorname{Re}\left(s_{j} a_{1 j}\right),
\end{aligned}
$$

e pela definição de B e a desigualdade anterior,

$$
\operatorname{Re} g\left(S\left(P_{B}\left(a_{1}\right)\right)\right)>\left(1-\frac{\nu^{2}}{2}\right) \sum_{j \in B}\left|S^{t}(g)\left(e_{j}\right)\right|>\left(1-\frac{\nu^{2}}{2}\right)\left(1-\frac{3 \nu}{4}\right) .
$$

Usando a Desigualdade 8, obtemos finalmente

$$
\operatorname{Re} g\left(S\left(P_{B}\left(a_{1}\right)\right)\right)>\left(1-\nu^{2}\right)\left(1-\frac{\nu}{1+\nu}\right)=1-\nu .
$$

Em decorrência da desigualdade acima e do fato que $g \in \overline{S_{\mathbb{K}} F(E)} w^{*}$, um argumento análogo ao já utilizado nesta demonstração nos permite concluir que existe um elemento em $S_{\mathbb{K}} F(E)$, digamos 
$\lambda F(e)$, para certos $\lambda \in S_{\mathbb{K}}$ e $e \in E$, tal que $\operatorname{Re} \lambda F(e)\left(S\left(P_{B}\left(a_{1}\right)\right)\right)>1-\nu$, ou seja,

$$
\operatorname{Re} F(e)\left(S\left(\lambda P_{B}\left(a_{1}\right)\right)\right)>1-\nu .
$$

Notemos que se $k \in B$, então $a_{1 k}=\overline{\lambda_{k}} \neq 0$, e portanto $B=\operatorname{supp} \lambda P_{B}\left(a_{1}\right)$. Tendo em vista (3.3.6), pelo Lema 3.3 .11 obtemos

$$
\left\|S\left(I-P_{B}\right)\right\| \leq \frac{\epsilon}{4}
$$

Agora já temos tudo o que é preciso para mostrar que o operador $U \doteq S M \in B_{\mathcal{L}\left(c_{0}, Y\right)}$ satisfaz a $\|U-T\|<\epsilon$ e $\|U(a)\|=1$ (já vimos que $\left\|a-x_{0}\right\|<\epsilon$ ). Recordemos que o operador $M \in B_{\mathcal{L}\left(c_{0}, Y\right)}$ é dado por

$$
M(x)=\left(a_{2 n} \overline{a_{1 n}} x_{n}\right)_{n \in \mathbb{N}}, \text { para todo } x \in c_{0} .
$$

Como

$$
\left(M\left(a_{1}\right)\right)_{n}= \begin{cases}0, & \text { se } n \notin A \\ a_{2 n} \lambda_{n} \overline{\lambda_{n}}=a_{2 n}, & \text { se } n \in A\end{cases}
$$

e $a_{2 n}=0$ para todo $n \in \mathbb{N} \backslash A$, concluímos que $M\left(a_{1}\right)=a_{2}$. Logo,

$$
U\left(a_{1}\right)=S\left(M\left(a_{1}\right)\right)=S\left(a_{2}\right)
$$

e por (3.3.19), $g\left(U\left(a_{1}\right)\right)=g\left(S\left(a_{2}\right)\right)=1$. Em particular, $\left\|U\left(a_{1}\right)\right\|=\|U\|=1$, isto é, $U$ atinge sua norma em $a_{1}$. Temos ainda que para todo $x \in c_{0}$,

$$
\left(M\left(P_{A}(x)\right)\right)_{n}=\left(P_{A}(M(x))\right)_{n}= \begin{cases}0, & \text { se } n \notin A \\ a_{2 n} \overline{a_{1 n}} x_{n}, & \text { se } n \in A\end{cases}
$$

isto é, $M P_{A}=P_{A} M$, o que implica

$$
U P_{A}=(S M) P_{A}=S\left(M P_{A}\right)=S\left(P_{A} M\right)=\left(S P_{A}\right) M=S M=U .
$$

Como $P_{A}(a)=a_{1}, U$ atinge sua norma também em $a \in S_{c_{0}}$. Resta provar que $\|U-T\|<\epsilon$. Para isso, vamos começar estimando a norma de $S P_{B}(M-I) \in \mathcal{L}\left(c_{0}, Y\right)$.

Seja $x \in B_{c_{0}}$. Temos que $(M-I)(x)=\left(a_{2 n} \overline{a_{1 n}} x_{n}-x_{n}\right)_{n \in \mathbb{N}} \mathrm{e}$

$$
\left(P_{B}(M-I)(x)\right)_{n}=\left(P_{B}(M-I) P_{B}(x)\right)_{n}= \begin{cases}0, & \text { se } n \notin B \\ a_{2 n} \overline{a_{1 n}} x_{n}-x_{n}, & \text { se } n \in B\end{cases}
$$

Portanto, $P_{B}(M-I)=P_{B}(M-I) P_{B}$ e em particular $\left[P_{B}(M-I)\right]\left(B_{c_{0}}\right)=\left[P_{B}(M-I)\right]\left(P_{B}\left(B_{c_{0}}\right)\right)$. Pelo Corolário 3.3.13,

$$
\left\|P_{B}(M-I)\right\|=\sup _{y \in V_{B}}\left\|P_{B}(M-I)(y)\right\|,
$$

em que $V_{B}=\left\{y \in P_{B}\left(B_{c_{0}}\right):\left|y_{i}\right|=1\right.$ para todo $\left.i \in B\right\}$. Mas, se $y \in V_{B}$,

$$
\left\|P_{B}(M-I)(y)\right\|=\max _{k \in B}\left|a_{2 k} \overline{a_{1 k}} y_{k}-y_{k}\right|=\max _{k \in B}\left|a_{2 k} \overline{a_{1 k}}-1\right| .
$$


Então $\left\|P_{B}(M-I)\right\|=\max _{k \in B}\left|a_{2 k} \overline{a_{1 k}}-1\right|=\max _{k \in B}\left|\overline{a_{2 k}} a_{1 k}-1\right|$. Como $B \subset A$, por (3.3.28) concluímos que $\left(k \in B \Rightarrow s_{k}=\overline{a_{2 k}}\right)$. Logo,

$$
\left\|P_{B}(M-I)\right\|=\max _{k \in B}\left|s_{k} a_{1 k}-1\right| .
$$

Utilizando agora (3.3.13), $\left|1-s_{k} a_{1 k}\right| \leq \sqrt{2\left(1-\operatorname{Re}\left(s_{k} a_{1 k}\right)\right)}$ para todo $k \in \mathbb{N}$. Se $k \in B$, teremos ainda $\operatorname{Re}\left(s_{k} a_{1 k}\right)>1-\frac{\nu^{2}}{2}$, donde vem

$$
\begin{aligned}
|| P_{B}(M-I)|| & =\max _{k \in B}\left|s_{k} a_{1 k}-1\right| \\
& \leq \max _{k \in B} \sqrt{2\left(1-\operatorname{Re}\left(s_{k} a_{1 k}\right)\right)}<\nu .
\end{aligned}
$$

Finalmente,

$$
\left\|S P_{B}(M-I)\right\| \leq\left\|P_{B}(M-I)\right\|<\nu .
$$

Notemos também que devido à (3.3.23),

$$
\|S-T\| \leq 2 \epsilon_{1}+\frac{\nu^{3}}{4}<2 \epsilon_{1}+\frac{\nu}{2}
$$

Agora vejamos que

$$
\begin{aligned}
\|S(M-I)\| & =\left\|S P_{B}(M-I)-S P_{B}(M-I)+S(M-I)\right\| \\
& \leq\left\|S P_{B}(M-I)\right\|+\left\|S\left(I-P_{B}\right)(M-I)\right\| \\
& (3.3 .31) \\
& <\nu+\left\|S\left(I-P_{B}\right)\right\| \cdot\|M-I\| \\
& \leq \nu+2\left\|S\left(I-P_{B}\right)\right\| \\
& (3.3 .30) \\
& \leq \nu+\frac{\epsilon}{2} .
\end{aligned}
$$

Enfim obtemos

$$
\begin{aligned}
\|U-T\| & \leq\|U-S\|+\|S-T\| \\
& =\|S(M-I)\|+\|S-T\| \\
& (3.3 .32) \\
< & \nu+\frac{\epsilon}{2}+2 \epsilon_{1}+\frac{\nu}{2} \\
& =\frac{3 \nu}{2}+\frac{\epsilon}{2}+2 \epsilon_{1},
\end{aligned}
$$

e pela Desigualdade $9,\|U-T\|<\epsilon$, encerrando a demonstração deste subcaso e do Teorema.

Observemos que devido à Proposição 3.3 .8 o espaço de Banach $c_{0}$ poderia ser substituído por $\ell_{\infty}^{n}$, para todo $n \in \mathbb{N}$, no enunciado do Teorema acima.

Mostraremos através do exemplo abaixo que os espaços de Banach $Y$ que satisfazem a propriedade $\beta$ de Lindenstrauss e os espaços uniformemente convexos cumprem as hipóteses do Teorema 3.3.15. Portanto, embora estes resultados já sejam conhecidos devido ao Teorema 3.3.7 e a [Kim13], o Teorema 3.3.15 implica por si só que o par $\left(c_{0}, Y\right)$ satisfaz a BPBp se $Y$ for uniformemente convexo 
ou se satisfizer a propriedade $\beta$.

Exemplo 3.3.16. Satisfazem as hipóteses do Teorema 3.3.15:

(a) Os espaços de Banach $Y$ que satisfazem a propriedade $\beta$ de Lindenstrauss.

Se $Y$ satisfaz a propriedade $\beta$, existem conjuntos $\left\{y_{\alpha}: \alpha \in \Lambda\right\} \subset S_{Y},\left\{f_{\alpha}: \alpha \in \Lambda\right\} \subset S_{Y^{*}} \mathrm{e}$ um real $\rho \in[0,1)$ que cumprem a Definição 3.1.10. Neste caso, basta tomar $E=\emptyset$ e os conjuntos e número real dados acima como os elementos do enunciado do Teorema 3.3.15. A função $F$ será a função vazia. As condições (I), (II) e (V) são satisfeitas devido às propriedades análogas da propriedade $\beta$ e as condições (III) e (IV) são satisfeitas por vacuidade.

(b) Os espaços de Banach $Y$ uniformemente convexos.

No Exemplo 3.1.9 vimos que tomando $E=S_{Y}$ e, para cada $e \in S_{Y}$, um funcional $f_{e} \in S_{Y^{*}}$ tal que $f_{e}(e)=1$, a família $E$ é fortemente e uniformemente exposta por $F: E \ni e \mapsto f_{e} \in S_{Y^{*}}$. Consideremos ainda $I=\emptyset$ e $\rho=0$. Dados estes elementos, já temos que a condição (III) é verdadeira. A condição $(\mathrm{V})$ também será verdadeira, pois dado qualquer $y \in Y \backslash\{0\}$, o funcional $f_{\frac{y}{\|y\|}}$ é tal que

$$
f_{\frac{y}{\|y\|}}(y)=f_{\frac{y}{\|y\|}}\left(\|y\| \frac{y}{\|y\|}\right)=\|y\| .
$$

As condições (I), (II) e (IV) são válidas por vacuidade.

Corolário 3.3.17. Se $Y$ é um espaço de Banach uniformemente convexo ou que satisfaz a propriedade $\beta$ de Lindenstrauss, o par $\left(c_{0}, Y\right)$ satisfaz a BPBp.

O Teorema 3.3.15 também nos concede pares $\left(c_{0}, Y\right)$ de espaços de Banach satisfazendo a BPBp que antes não eram conhecidos. De fato, o resultado de [AGKM17] apresentado em seguida garante a existência espaços de Banach que não são uniformemente convexos nem satisfazem a propriedade $\beta$ mas que cumprem as hipóteses do Teorema 3.3.15. Antes de apresentar este resultado, precisamos de algumas definições e de uma propriedade de topologia.

Definição 3.3.18. Um sistema biortogonal em um espaço de Banach $X$ é um subconjunto $\left\{\left(x_{i}, f_{i}\right)\right.$ : $i \in I\} \subset X \times X^{*}$ tal que $f_{i}\left(x_{j}\right)=\delta_{i j}$ para todos $i, j \in I$.

O sistema biortogonal $\left\{\left(x_{i}, f_{i}\right): i \in I\right\}$ é dito limitado se $\left(\left\|x_{i}\right\| \cdot\left\|f_{i}\right\|\right)_{i \in I}$ é uma família limitada, e é dito total se $\left\{f_{i}: i \in I\right\}$ separa pontos de $X$.

Afirmamos agora que se $V$ é um espaço topológico e $A, B \subset V$ são subconjuntos fechados de $V$, então $\partial(A \cap B)=(A \cap \partial B) \cup(B \cap \partial A)$. De fato,

$$
\begin{aligned}
\partial(A \cap B) & \doteq \overline{(A \cap B)} \backslash(A \cap B)^{\mathrm{o}} \\
& =(A \cap B) \cap\left((A \cap B)^{\mathrm{o}}\right)^{\complement} \\
& =(A \cap B) \cap\left(A^{\mathrm{o}} \cap B^{\mathrm{o}}\right)^{\complement} \\
& =(A \cap B) \cap\left(\left(A^{\mathrm{o}}\right)^{\complement} \cup\left(B^{\mathrm{o}}\right)^{\complement}\right) \\
& =(A \cap B) \cap\left(\left(\partial A \cup A^{\complement}\right) \cup\left(\partial B \cup B^{\complement}\right)\right) \\
& =\left((A \cap B) \cap\left(\partial A \cup A^{\complement}\right)\right) \cup\left((A \cap B) \cap\left(\partial B \cup B^{\complement}\right)\right) \\
& =(A \cap B \cap \partial A) \cup(A \cap B \cap \partial B)
\end{aligned}
$$




$$
=(\partial A \cap B) \cup(\partial B \cap A) .
$$

A demonstração da letra (d) do Teorema abaixo também faz uso de um resultado de J. P. Moreno, apresentado em [Mor97], que afirma que se um espaço de Banach de dimensão maior que 1 possui pontos LUR (vide Definição 1.5.5), então não satisfaz a propriedade $\beta$ de Lindenstrauss. Não apresentamos a demonstração deste resultado aqui pois é muito técnica e possui detalhes que fogem do escopo deste trabalho.

Teorema 3.3.19 ([AGKM17]). Sejam (X,\|.\|) um espaço de Banach, I um conjunto não-vazio e $\left\{\left(x_{i}, f_{i}\right): i \in I\right\}$ um sistema biortogonal limitado em $(X,\|\|$.$) tal que \left\|x_{i}\right\|=1$ para todo $i \in I$. Sejam ainda $K \doteq \sup \left\{\left\|f_{i}\right\|: i \in I\right\}, M>K e$

$$
B \doteq M B_{X} \cap\left\{x \in X:\left|f_{i}(x)\right| \leq 1 \text { para todo } i \in I\right\}
$$

Então as seguintes afirmações são satisfeitas:

(a) O conjunto $B$ é a bola unitária fechada de uma nova norma ||$|\cdot|||$ em $X$, equivalente à norma original $\|$.$\| , que satisfaz a$

$$
\frac{1}{K}|\|x\|||\leq\|x\| \leq M|\|x \mid\|, \text { para todo } x \in X \text {. }
$$

(b) Se X é uniformemente convexo, então o espaço de Banach $Y \doteq(X,|||\cdot|||)$ satisfaz as hipóteses do Teorema 3.3.15.

(c) Se $\operatorname{dim} X>1$, então $Y$ não é estritamente convexo.

(d) Se o sistema biortogonal $\left\{\left(x_{i}, f_{i}\right): i \in I\right\}$ não é total e $X$ é uniformemente convexo com $\operatorname{dim} X>1$, então $Y$ não satisfaz a propriedade $\beta$ de Lindenstrauss.

Demonstração. No decorrer desta demonstração, dependendo do contexto, a notação $X$ se refere ao espaço de Banach $(X,\|\|$.$) ou apenas ao próprio conjunto X$. Nesse espírito, a bola unitária fechada de $(X,\|\|$.$) será denotada simplesmente por B_{X}$.

Observemos inicialmente que $I \neq \emptyset$ implica que $K \geq 1$, uma vez que $f_{i}\left(x_{i}\right)=1=\left\|x_{i}\right\|$ para todo $i \in I$.

(a) Notemos que o conjunto $B$ é fechado (pois é interseção de conjuntos fechados), limitado, equilibrado e convexo. Afirmamos que $0 \in B^{\circ}$. De fato, uma vez que $\frac{1}{K}<M$, temos $\frac{1}{K} B_{X} \subset M B_{X}$, e dado $y \in \frac{1}{K} B_{X}$, para todo $i \in I$,

$$
\left|f_{i}(y)\right| \leq \frac{1}{K} \sup \left\{|| f_{i} \|: i \in I\right\}=1,
$$

e então $\frac{1}{K} B_{X} \subset B$. Em particular, $\frac{1}{K} B_{X}^{\circ} \subset B^{\circ}$, donde segue a conclusão desejada. Portanto, pela Proposição 1.1.6, existe uma norma |||.||| em $X$ para a qual $B$ é a bola unitária fechada. Como já 
mostrado, temos ainda que

$$
\frac{1}{K} B_{X} \subset B \subset M B_{X}
$$

Por (3.3.33), dado $x \in X \backslash\{0\}$, vale que $\frac{1}{K} \frac{x}{\|x\|} \in B$, ou seja, \|\|$\frac{1}{K} \frac{x}{\|x\|}\|\| \leq 1$. Portanto, $\frac{1}{K} \mid\|x\|\|\leq\| x \|$ para todo $x \in X$. Novamente por (3.3.33), dado $x \in X \backslash\{0\}$, vale que $\frac{x}{\|x\| \|} \in M B_{X}$, ou seja, $\left\|\frac{x}{\||| x \mid\|}\right\| \leq M$ e, portanto, $\|x\| \leq M\|\| x\|\|$ para todo $x \in X$. Juntando as conclusões deste parágrafo, obtemos que

$$
\frac{1}{K}\|x\| \mid\|\| x\|\leq M\|\|x\| \| \text { para todo } x \in X
$$

como queríamos demonstrar.

(b) Temos que $B_{Y}=B$, conforme o item anterior. Como $\|$.$\| e |||.||| são equivalentes, a topologia$ dos espaços $(X,\|\|$.$) e Y$ é a mesma. Em particular, a fronteira de $B$ relativa à norma ||$|\cdot| \|$, que é $S_{Y}$, é igual à fronteira $\partial B$ de $B$ relativa à norma $\|$.$\| . Como M B_{X}$ e $\left\{x \in X:\left|f_{i}(x)\right| \leq 1\right.$ para todo $i \in$ $I\}$ são conjuntos fechados, da discussão que antecede o enunciado deste Teorema concluímos que

$$
\begin{array}{r}
S_{Y}=\partial B=\left(M S_{X} \cap\left\{x \in X:\left|f_{i}(x)\right| \leq 1 \text { para todo } i \in I\right\}\right) \\
\bigcup\left(M B_{X} \cap \partial\left\{x \in X:\left|f_{i}(x)\right| \leq 1 \text { para todo } i \in I\right\}\right) .
\end{array}
$$

Vamos mostrar que $\partial\left\{x \in X:\left|f_{i}(x)\right| \leq 1\right.$ para todo $\left.i \in I\right\}=\left\{x \in X: \sup \left\{\left|f_{i}(x)\right|: i \in I\right\}=1\right\}$.

Seja $x_{0} \in\left\{x \in X: \sup \left\{\left|f_{i}(x)\right|: i \in I\right\}=1\right\}$. Dado $r>0$, existe $i_{0} \in I$ tal que $\left|f_{i_{0}}\left(x_{0}\right)\right|>1-\frac{r}{2}$. Consideremos ainda $\lambda_{0} \in S_{\mathbb{K}}$ satisfazendo a $f_{i_{0}}\left(x_{0}\right)=\left|f_{i_{0}}\left(x_{0}\right)\right| \lambda_{0}$. Então $x_{0}+\frac{\lambda_{0} x_{i_{0}} r}{2} \in B_{r}\left(x_{0}\right)$ e

$$
\left|f_{i_{0}}\left(x_{0}+\frac{\lambda_{0} x_{i_{0}} r}{2}\right)\right|=\left|\lambda_{0}\right|\left(\frac{r}{2}+\left|f_{i_{0}}\left(x_{0}\right)\right|\right)>1
$$

portanto $B_{r}\left(x_{0}\right) \cap\left\{x \in X:\left|f_{i}(x)\right| \leq 1 \text { para todo } i \in I\right\}^{\complement} \neq \emptyset$. Como também temos que $x_{0} \in\{x \in$ $X:\left|f_{i}(x)\right| \leq 1$ para todo $\left.i \in I\right\}$, segue que $\left\{x \in X: \sup \left\{\left|f_{i}(x)\right|: i \in I\right\}=1\right\} \subset \partial\left\{x \in X:\left|f_{i}(x)\right| \leq\right.$ 1 para todo $i \in I\}$. Consideremos agora $y_{0} \in \partial\left\{x \in X:\left|f_{i}(x)\right| \leq 1\right.$ para todo $\left.i \in I\right\}$. É claro que nesse caso $\sup \left\{\left|f_{i}\left(y_{0}\right)\right|: i \in I\right\} \leq 1$. Suponhamos por absurdo que $r \doteq \sup \left\{\left|f_{i}\left(y_{0}\right)\right|: i \in I\right\}<1$. Dado $x \in B_{\frac{(1-r)}{K}}\left(y_{0}\right)$ teremos, para todo $i \in I$,

$$
\left|f_{i}(x)\right| \leq\left|f_{i}\left(x-y_{0}\right)\right|+\left|f_{i}\left(y_{0}\right)\right| \leq K \frac{(1-r)}{K}+r=1 .
$$

Portanto, $B_{\frac{(1-r)}{K}}\left(y_{0}\right) \cap\left\{x \in X:\left|f_{i}(x)\right| \leq 1 \text { para todo } i \in I\right\}^{\complement}=\emptyset$, absurdo. Logo, $\sup \left\{\left|f_{i}\left(y_{0}\right)\right|: i \in\right.$ $I\}=1$, donde segue a conclusão desejada. 
Assim,

$$
\begin{gathered}
S_{Y}=\left(M S_{X} \cap\left\{x \in X:\left|f_{i}(x)\right| \leq 1 \text { para todo } i \in I\right\}\right) \\
\bigcup\left(M B_{X} \cap\left\{x \in X: \sup \left\{\left|f_{i}(x)\right|: i \in I\right\}=1\right\}\right) .
\end{gathered}
$$

Definamos

$$
E \doteq M S_{X} \cap\left\{x \in X:\left|f_{i}(x)\right| \leq 1 \text { para todo } i \in I\right\}
$$

Pelo Corolário 1.1.16, para cada $x \in E$ existe $f_{x} \in S_{X^{*}}$ tal que $f_{x}(x)=\|x\|=M$. Consideremos a função $F: E \rightarrow X^{*}$ definida por

$$
F(x)=\frac{f_{x}}{M}, \text { para cada } x \in E
$$

Como as normas ||$.||$ e |||.||| são equivalentes, o conjunto dos funcionais lineares e contínuos definidos em $X$ e em $Y$ são iguais. Ou seja, vistos apenas como conjuntos, $X^{*}=Y^{*}$. Além disso, devido à desigualdade entre as normas vista no item (a),

$$
\frac{|\|f|\||}{M} \leq\|f\| \leq K \mid\|f\| \| \text { para todo } f \in X^{*}=Y^{*}
$$

Para cada $x \in E,\|F(x)\|=\frac{1}{M}$, e então $\|F(x)\| \| \leq 1$. Além disso, $F(x)(x)=1$. Portanto, $F$ é função de $E$ em $S_{Y^{*}}$. Mostraremos em seguida que $Y$ satisfaz as hipóteses do Teorema 3.3 .15 com os elementos $\rho \doteq \frac{1}{M}, E \subset S_{Y}, F: E \rightarrow S_{Y^{*}},\left\{x_{i}: i \in I\right\}$ e $\left\{f_{i}: i \in I\right\}$.

Para começar, observemos que $x_{i} \in S_{Y}$ para todo $i \in I$, já que $x_{i} \in\left(M B_{X} \cap\{x \in X\right.$ : $\left.\left.\sup \left\{\left|f_{i}(x)\right|: i \in I\right\}=1\right\}\right)$. Além disso, se $y \in S_{Y}$, então $\left|f_{i}(y)\right| \leq 1$ para todo $i \in I$. Como também temos $f_{i}\left(x_{i}\right)=1$, segue que $f_{i} \in S_{Y^{*}}$ para todo $i \in I$. As condições (I) e (II) do Teorema 3.3.15 são satisfeitas com $\rho=\frac{1}{M}$, uma vez que $\left\{\left(x_{i}, f_{i}\right): i \in I\right\}$ é um sistema biortogonal para $X$.

Vamos mostrar que vale a condição $(\mathrm{V})$. Dado $y \in S_{Y}$, temos que $\left(\sup \left\{\left|f_{i}(y)\right|: i \in I\right\}=1\right)$ ou $(y \in E)$. No primeiro caso, a conclusão segue imediatamente, e no segundo caso é consequência do fato que $F(y)(y)=1$, por construção. Portanto,

$$
\||| y \mid\|=\sup \left\{|f(y)|: f \in F(E) \cup\left\{f_{i}: i \in I\right\}\right\} \text {, para todo } y \in X .
$$

Agora mostraremos a condição (III). Como $X$ é uniformemente convexo, para cada $\epsilon>0$ existe $\delta(\epsilon)>0$ tal que

$$
x, y \in B_{X}, \frac{\|x+y\|}{2}>1-\delta(\epsilon) \Rightarrow\|x-y\|<\epsilon .
$$

Afirmamos que dado $\epsilon>0$,

$$
\left(y \in B_{X}, x \in S_{X}, f \in S_{X^{*}}, f(x)=1, \operatorname{Re} f(y)>1-2 \delta(\epsilon)\right) \Rightarrow\|x-y\|<\epsilon .
$$


De fato, dados $y \in B_{X}, x \in S_{X}$ e $f \in S_{X^{*}}$ tais que $f(x)=1$ e $\operatorname{Re} f(y)>1-2 \delta(\epsilon)$, vale que

$$
\frac{\|x+y\|}{2} \geq \frac{|f(x+y)|}{2} \geq \operatorname{Re} \frac{f(x+y)}{2}=\frac{1}{2}+\operatorname{Re} \frac{f(y)}{2}>1-\delta(\epsilon),
$$

e isso implica que $\|x-y\|<\epsilon$, donde obtemos (3.3.34). Considere então $\epsilon>0, e \in E$ e $y \in B_{Y}=B$. Teremos que $\frac{y}{M} \in B_{X}, \frac{e}{M} \in S_{X}, M F(e) \in S_{X^{*}}, M F(e)\left(\frac{e}{M}\right)=1$ e $M F(e)\left(\frac{y}{M}\right)=F(e)(y)$. Portanto, por (3.3.34),

$$
\left(e \in E, y \in B_{Y}=B, \operatorname{Re} F(e)(y)>1-2 \delta(\epsilon)\right) \Rightarrow\left\|\frac{e}{M}-\frac{y}{M}\right\|<\epsilon \Rightarrow \mid\|y-e\| \|<K M \epsilon,
$$

o que implica que $E$ é fortemente e uniformemente exposta por $\mathrm{F}$.

Por fim, mostraremos que vale a condição (IV). Para isso, basta observar que dados $e \in E$ e $i \in I$,

$$
\left|F(e)\left(x_{i}\right)\right|=\left|\frac{f_{e}}{M}\left(x_{i}\right)\right| \leq \frac{1}{M}\left\|f_{e}\right\| \cdot\left\|x_{i}\right\|=\frac{1}{M}=\rho<1 .
$$

(c) Tomemos $i_{0} \in I(\neq \emptyset)$. Como $\operatorname{dim} X>1$, existe $y \in \operatorname{Ker} f_{i_{0}} \cap S_{X}$. Para todo $t \in \mathbb{K}$, vale que $\left\|x_{i_{0}}+t y\right\| \leq 1+|t|$. Seja $t_{0} \in \mathbb{K} \backslash\{0\}$ satisfazendo a $\left|t_{0}\right|<\min \left\{M-1, \frac{1}{K}\right\}$. Então $\left\|x_{i_{0}}+t_{0} y\right\|<M$, ou seja, $\left(x_{i_{0}}+t_{0} y\right) \in M B_{X}$. Além disso, se $i \in I \backslash\left\{i_{0}\right\}$,

$$
\left|f_{i}\left(x_{i_{0}}+t_{0} y\right)\right|=\left|f_{i}\left(t_{0} y\right)\right| \leq|| f_{i}|| \cdot\left|t_{0}\right| \leq K\left|t_{0}\right|<1
$$

e $\left|f_{i_{0}}\left(x_{i_{0}}+t_{0} y\right)\right|=1$. Portanto, $\sup \left\{\left|f_{i}\left(x_{i_{0}}+t_{0} y\right)\right|: i \in 1\right\}=1$, o que implica que $\left(x_{i_{0}}+t_{0} y\right) \in S_{Y}$, e analogamente $\left(x_{i_{0}}-t_{0} y\right) \in S_{Y}$. Como $\left(x_{i_{0}}+t_{0} y\right) \neq\left(x_{i_{0}}-t_{0} y\right)$ e

$$
\frac{1}{2}\left(\left(x_{i_{0}}+t_{0} y\right)+\left(x_{i_{0}}-t_{0} y\right)\right)=x_{i_{0}} \in S_{Y}
$$

concluímos que $Y$ não é estritamente convexo.

(d) Como $X$ é uniformemente convexo, dado $\epsilon>0$ existe $\delta(\epsilon)>0$ tal que

$$
x, y \in B_{X}, \frac{\|x+y\|}{2}>1-\delta(\epsilon) \Rightarrow\|x-y\|<\epsilon
$$

Por hipótese, o sistema biortogonal $\left\{\left(x_{i}, f_{i}\right): i \in I\right\}$ não é total, então existe $e \in S_{Y}$ tal que $f_{i}(e)=0$ para todo $i \in I$. Como

$$
S_{Y}=E \cup\left(M B_{X} \cap\left\{x \in X: \sup \left\{\left|f_{i}(x)\right|: i \in I\right\}=1\right\}\right),
$$

concluímos que $e \in E$. Vamos mostrar que $e$ é ponto LUR de $Y$.

Para cada $\epsilon>0$, seja $\delta_{1}(\epsilon)$ um real positivo tal que $\delta_{1}(\epsilon)<\min \left\{\delta(\epsilon), \frac{1}{2}\right\}$. Sejam $\epsilon>0$ e $y \in S_{Y}$ 
satisfazendo a

$$
\left\|\frac{y+e}{2}\right\|>1-\delta_{1}(\epsilon) .
$$

Pelo item (b), existe $G \in F(E) \cup\left\{f_{i}: i \in I\right\}$ tal que $\left|G\left(\frac{y+e}{2}\right)\right|>1-\delta_{1}(\epsilon)$. Se tivéssemos $G=f_{j}$ para algum $j \in I$, então

$$
\left|\frac{f_{j}(y+e)}{2}\right|=\left|\frac{f_{j}(y)}{2}\right|>1-\delta_{1}(\epsilon)>\frac{1}{2}
$$

absurdo, uma vez que $\left|f_{j}(y)\right| \leq 1$. Portanto, $G \in F(E)$, e assim $M G \in S_{X^{*}}$ por construção. Observemos que $\frac{y}{M}, \frac{e}{M} \in B_{X}$ e

$$
\left\|\frac{(y / M)+(e / M)}{2}\right\| \geq\left|(M G)\left(\frac{y / M+e / M}{2}\right)\right|>1-\delta_{1}(\epsilon)>1-\delta(\epsilon),
$$

o que implica $\left\|\frac{y-e}{M}\right\|<\epsilon$, ou seja, $\|y-e\| \mid<(M K) \epsilon$. Dessa forma, concluímos que $e \in S_{Y}$ é ponto LUR de $Y$.

Como já afirmado anteriormente, J. P. Moreno mostrou em [Mor97] que espaços de Banach de dimensão maior que 1 contendo pontos LUR não satisfazem a propriedade $\beta$, donde segue que $Y$ não satisfaz a propriedade $\beta$.

Seja $(X,\|\|$.$) um espaço de Banach uniformemente convexo com \operatorname{dim} X>1$. Consideremos $x_{0} \in S_{X}$ arbitrário e $f_{0} \in S_{X *}$ satisfazendo a $f_{0}\left(x_{0}\right)=1$ (Corolário 1.1.16). Então $\left\{\left(x_{0}, f_{0}\right)\right\}$ é um sistema biortogonal limitado em $X$. Além disso, como $\operatorname{dim} X>1$, este sistema biortogonal não é total. Portanto, dado $n \in \mathbb{N}$, podemos aplicar o Teorema $3.3 .19 \mathrm{em}(X,\|\|$.$) para M_{n} \doteq 1+\frac{1}{n}>$ $1=\left\|f_{0}\right\|$ e obter um espaço de Banach $Y \doteq\left(X,\|\cdot\|_{n}\right)$ que não é uniformemente convexo (pois não é estritamente convexo) nem satisfaz a propriedade $\beta$ de Lindenstrauss, mas obedece as hipóteses do Teorema 3.3.15. Além disso, vale que

$$
B_{Y}=\left(1+\frac{1}{n}\right) B_{X} \cap\left\{x \in X:\left|f_{0}(x)\right| \leq 1\right\}
$$

e

$$
\|x\|_{n} \leq\|x\| \leq\left(1+\frac{1}{n}\right)\|x\|_{n}, \text { para todo } x \in X .
$$

O Corolário seguinte resume a discussão acima:

Corolário 3.3.20 ([AGKM17]). Seja $(X,\|\|$.$) um espaço de Banach uniformemente convexo com$ $\operatorname{dim} X>1$. Para todo $n \in \mathbb{N}$, existe uma norma $\|.\|_{n}$ em $X$ equivalente à $\|$.$\| e tal que:$

(i) $\left(X,\|\cdot\|_{n}\right)$ satisfaz as hipóteses do Teorema 3.3.15.

(ii) $\left(X,\|.\|_{n}\right)$ não é uniformemente convexo nem satisfaz a propriedade $\beta$ de Lindenstrauss.

(iii) $\|x\|_{n} \leq\|x\| \leq\left(1+\frac{1}{n}\right)\|x\|_{n}$, para todo $x \in X$. 
Concluímos assim que de fato o Teorema 3.3 .15 concede exemplos de pares $\left(c_{0}, Y\right)$ de espaços de Banach satisfazendo a BPBp que antes não eram conhecidos.

O Teorema 3.3.15 dá uma condição suficiente sobre espaços de Banach $Y$ para que o par $\left(c_{0}, Y\right)$ satisfaça a BPBp, mas não existe garantia de que esta condição seja também necessária. Entretanto, Kim mostrou que no caso real, se $Y$ é estritamente convexo, podemos caracterizar os pares $\left(c_{0}, Y\right)$ de espaços de Banach que satisfazem a BPBp, da forma que veremos abaixo.

Teorema 3.3.21 ([Kim13]). Sejam $X$ o espaço de Banach real $c_{0}, \ell_{\infty}$ ou $\ell_{\infty}^{n}$, para $n \geq 2$, e $Y$ um espaço de Banach real estritamente convexo. Se $(X, Y)$ satisfaz a BPBp, então $Y$ é uniformemente convexo.

Demonstração. Faremos a demonstração para o caso $X=c_{0}$. Os demais casos seguem por argumentos análogos.

Por hipótese, para todo $\epsilon>0$ existe $\eta(\epsilon)>0$ satisfazendo a Definição 3.3.1. Suponhamos que $Y$ não é uniformemente convexo. Então existem sequências $\left(x_{n}\right)_{n \in \mathbb{N}}$ e $\left(y_{n}\right)_{n \in \mathbb{N}}$ em $S_{Y}$ tais que $\frac{1}{2}\left\|x_{n}+y_{n}\right\| \rightarrow 1$ e $\left\|x_{n}-y_{n}\right\| \nrightarrow 0$ (vide Proposição 1.5.4). Tomando uma subsequência se necessário, podemos supor que existe $\epsilon_{0} \in(0,1)$ tal que $\left\|x_{n}-y_{n}\right\| \geq \epsilon_{0}$ para todo $n \in \mathbb{N}$.

Definamos, para cada $n \in \mathbb{N}$, o operador $T_{n} \in \mathcal{L}\left(c_{0}, Y\right)$ dado por

$$
T_{n}(z)=z_{1} \frac{\left(x_{n}+y_{n}\right)}{2}+z_{2} \frac{\left(x_{n}-y_{n}\right)}{2} \text {, para todo } z \in c_{0}
$$

Notemos que todos $n \in \mathbb{N}$ e $z \in c_{0}$,

$$
\left\|T_{n}(z)\right\|=\frac{1}{2}\left\|\left(z_{1}+z_{2}\right) x_{n}+\left(z_{1}-z_{2}\right) y_{n}\right\| \leq \frac{\left|z_{1}+z_{2}\right|}{2}+\frac{\left|z_{1}-z_{2}\right|}{2} .
$$

Como por hipótese $c_{0}$ é espaço normado sobre $\mathbb{R}$, as possibilidades para a soma $\left|z_{1}+z_{2}\right|+\left|z_{1}-z_{2}\right|$ são $\pm 2 z_{1}$ e $\pm 2 z_{2}$. Portanto, $\left\|T_{n}\right\| \leq 1$, e ainda $\left\|T_{n}\left(e_{1}+e_{2}\right)\right\|=\left\|x_{n}\right\|=1$. Logo, $T_{n} \in S_{\mathcal{L}\left(c_{0}, Y\right)}$ para todo $n \in \mathbb{N}$.

Observemos agora que $\lim _{n \rightarrow \infty}\left\|T_{n}\left(e_{1}\right)\right\|=1$. Seja $m \in \mathbb{N}$ tal que $\left\|T_{m}\left(e_{1}\right)\right\|>1-\eta\left(\epsilon_{0} / 2\right)$. Então existem $\tilde{T} \in S_{\mathcal{L}\left(c_{0}, Y\right)}$ e $\tilde{z} \in S_{c_{0}}$ satisfazendo a

$$
\|\tilde{T}(\tilde{z})\|=1,\left\|\tilde{T}-T_{m}\right\|<\frac{\epsilon_{0}}{2} \mathrm{e}\left\|\tilde{z}-e_{1}\right\|<\frac{\epsilon_{0}}{2} .
$$

Em particular, $\left|\tilde{z}_{n}\right|<\frac{\epsilon_{0}}{2}<1$ para todo $n \geq 2$. Pela Proposição $3.2 .3, \tilde{T}\left(e_{n}\right)=0$ para todo $n \geq 2$. Portanto,

$$
\tilde{T}\left(e_{1}+e_{2}\right)=\tilde{T}\left(e_{1}-e_{2}\right)=\tilde{T}\left(e_{1}\right)
$$

e assim,

$$
\begin{aligned}
\left\|x_{m}-y_{m}\right\| & =\left\|T_{m}\left(e_{1}+e_{2}\right)-T_{m}\left(e_{1}-e_{2}\right)\right\| \\
& \leq\left\|T_{m}\left(e_{1}+e_{2}\right)-\tilde{T}\left(e_{1}+e_{2}\right)\right\|+\left\|\tilde{T}\left(e_{1}+e_{2}\right)-T_{m}\left(e_{1}-e_{2}\right)\right\| \\
& =\left\|T_{m}\left(e_{1}+e_{2}\right)-\tilde{T}\left(e_{1}+e_{2}\right)\right\|+\left\|\tilde{T}\left(e_{1}-e_{2}\right)-T_{m}\left(e_{1}-e_{2}\right)\right\| \\
& \leq 2\left\|\tilde{T}-T_{m}\right\|<\epsilon_{0},
\end{aligned}
$$


absurdo. Logo, $Y$ é uniformemente convexo.

Como já sabíamos que se $Y$ é uniformemente convexo, então $\left(c_{0}, Y\right)$ e $\left(\ell_{\infty}^{n}, Y\right)$ satisfazem a BPBp, o Teorema acima permite concluir o Corolário seguinte.

Corolário 3.3.22. Seja $Y$ um espaço de Banach real estritamente convexo. O par $\left(c_{0}, Y\right)$ (ou $\left(\ell_{\infty}^{n}, Y\right)$, para $\left.n \geq 2\right)$ satisfaz a BPBp se, e somente se, $Y$ é uniformemente convexo.

O Corolário acima implica em particular que existem pares $(X, Y)$ de espaços de Banach tais que $\mathcal{N} \mathcal{A}(X, Y)$ é denso em $\mathcal{L}(X, Y)$ mas $(X, Y)$ não satisfaz a BPBp. De fato, seja $Y$ um espaço de Banach real estritamente convexo que não é uniformemente convexo. Pelo Corolário 3.3.22, $\left(\ell_{\infty}^{n}, Y\right)$ não satisfaz a BPBp, qualquer que seja $n \geq 2$. Porém, como os espaços $\ell_{\infty}^{n}$ têm dimensão finita, $\mathcal{N} \mathcal{A}\left(\ell_{\infty}^{n}, Y\right)$ é denso em $\mathcal{L}\left(\ell_{\infty}^{n}, Y\right)$ para todo $n \in \mathbb{N}$. 


\section{Referências Bibliográficas}

[AA96] M. D. Acosta e F. Aguirre. A new sufficient condition for the denseness of norm attaining operators. Rocky Mountain J. Math., 26:407-418, 1996. xii

[AAGM08] M. D. Acosta, R. M. Aron, D. García e M. Maestre. The Bishop-Phelps-Bollobás theorem for operators. J. Funct. Anal., 254:2780-2799, 2008. iii, v, xii, xiii, 45, 49, 52, $53,54,56,57,58$

$\left[\mathrm{ABGC}^{+} 14\right]$ M. D. Acosta, J. Becerra-Guerrero, Y. S. Choi, M. Ciesielski, S. K. Kim, H. J. Lee, M. L. Lourenço e M. Martín. The Bishop-Phelps-Bollobás property for operators between spaces of continuous functions. Nonlinear Anal., 95:323-332, 2014. xii

[ABGG $\left.{ }^{+} 15\right]$ M. D. Acosta, J. Becerra-Guerrero, D. García, S. K. Kim e M. Maestre. The BishopPhelps-Bollobás property: a finite dimensional approach. Publ. Res. Inst. Math. Sci., 51:173-190, 2015. xii, 57

[ACK $\left.{ }^{+} 15\right]$ R. Aron, Y. S. Choi, S. K. Kim, H. J. Lee e M. Martín. The Bishop-Phelps-Bollobás versions of Lindenstrauss properties A and B. Trans. Amer. Math. Soc., 367:60856101, 2015. 57

[Aco16] M. D. Acosta. The Bishop-Phelps-Bollobás property for operators on $C(K)$. Banach J. Math. Anal., 10(2):307-319, 2016. xii, 57

[ADSM19] M. D. Acosta, J. L. Dávila e M. Soleimani-Mourchehkhorti. Characterization of Banach spaces Y satisfying that the pair $\left(\ell_{\infty}^{4}, Y\right)$ has the Bishop-Phelps-Bollobás property for operators. J. Math. Anal. Appl., 470:690-715, 2019. xii, 57

[AGKM17] M. D. Acosta, D. García, S. K. Kim e M. Maestre. The Bishop-Phelps-Bollobás property for operators from $c_{0}$ into some Banach spaces. J. Math. Anal. Appl., 445:11881199, 2017. iii, v, xii, xiii, 45, 47, 52, 57, 58, 60, 62, 66, 76, 77, 81

[Bol70] B. Bollobás. An extension to the theorem of Bishop and Phelps. Bull. London Math. Soc., 2:181-182, 1970. xi, xii, 25, 41

[BP61] E. Bishop e R. R. Phelps. A proof that every Banach space is subreflexive. Bull. Amer. Math. Soc., 67:97-98, 1961. xi, 25, 28

[BP63] E. Bishop e R. R. Phelps. The support functionals of a convex set. Proc. Symp. Pure Math., 7:27-35, 1963. xii, 28, 29, 35, 37, 38

[BPT15] G. Botelho, D. Pellegrino e E. Teixeira. Fundamentos de Análise Funcional. Coleção Textos Universitários. SBM, 2015. 10, 15, 23

[Jam57] R. James. Reflexivity and the Supremum of Linear Functionals. Ann. of Math., 66(1):159-169, 1957. xi, 25

[Jam64] R. James. Characterizations of reflexivity. Studia Math., 23:205-216, 1964. xi, 25 
[Kim13] S. K. Kim. The Bishop-Phelps-Bollobás Theorem for operators from $c_{0}$ to uniformly convex spaces. Israel J. Math., 197:425-435, 2013. xii, xiii, 57, 75, 82

[KLL16] S. H. Kim, H. J. Lee e P. K. Lin. The Bishop-Phelps-Bollobás theorem for operators from $L_{\infty}(\mu)$ to uniformly convex spaces. J. Nonlinear Convex Anal., 17(2):243-249, 2016. xii, 57

[Lin63] J. Lindenstrauss. On operators which attain their norm. Israel J. Math., 1:139-148, 1963. xi, xiii, $45,47,48,49$

[Meg98] R. E. Megginson. An Introduction to Banach Space Theory. Springer New York, 1998. $1,12,15,26,28,29$

[Mor97] J. P. Moreno. Geometry of Banach spaces with $(\alpha, \beta)$-property or $(\beta, \epsilon)$-property. Rocky Mountain J. Math., 27:241-256, 1997. 77, 81

[Phe57] R. Phelps. Subreflexive normed linear spaces. Arch. Math. (Basel), 8:444-450, 1957. xi, 25,29

[Phe93] R. Phelps. Convex functions, Monotone Operators and Differentiability. SpringerVerlag, Berlin New York, 1993. 37

[Sch83] W. Schachermayer. Norm attaining operators and renorming of Banach spaces. Israel J. Math., 44(3):201-212, 1983. xi

[Wil04] S. Willard. General Topology (Dover Books on Mathematics). Dover Publications, 2004. 1,9 